Supporting information

\title{
Total Synthesis of (+)-Galbulin and Unnatural Lignans
}

Florian Clausen and Armido Studer

ORGANISCH-CHEMISCHES INSTITUT, Westfälische Wilhelms-Universität, Corrensstraße 40, 48149 Münster, Germany, studer@wwu.de

Table of contents

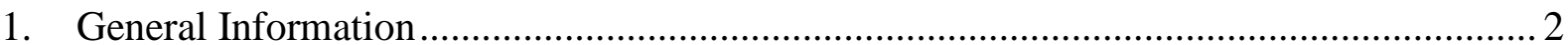

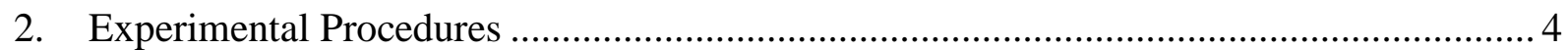

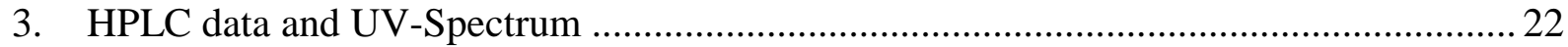

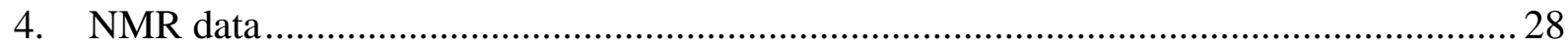




\section{General Information}

All reactions involving air or moisture sensitive reagents were carried out in flame-dried glass ware under argon atmosphere using standard SCHLENK techniques. Solvents used in reactions were either freshly distilled or obtained in extra-dry grade from commercial sources. Diethyl ether $\left(\mathrm{Et}_{2} \mathrm{O}\right)$ was refluxed over $\mathrm{K}$ and freshly distilled from K-Na-alloy (4:1) afterwards. Tetrahydrofuran (THF) was refluxed over $\mathrm{Na}$ and distilled from $\mathrm{K}$ afterwards. Acetone (99.8\%, Extra Dry, AcroSeal $\left.{ }^{\circledR}\right)$ and methanol (MeOH, 99.8\%, Extra Dry over Molecular Sieve, AcroSeal®) were purchased from ACROS ORGANICS. Solvents for flash chromatography (FC) were freshly distilled before use. $n$-Butyl lithium ( $n$-BuLi, $1.6 \mathrm{M}$ in hexanes) was purchased from ACROS ORGANICS. The boronic esters not listed in the experimental section were purchased from ABCR. All other chemicals were purchased from ABCR, Acros Organics, Alfa Aesar, Fluka, Sigma Aldrich and TCI and were used as received. Reactions requiring heating were conducted using an oil bath as heating source. Flash chromatography was performed on MERCK silica gel $60(40-63 \mu \mathrm{m})$ with an excess argon pressure up to 1.0 bar. MERCK silica gel 60 F254 plates were used for thin layer chromatography (TLC) using UV light (254/366 nm), $\mathrm{KMnO}_{4}\left(1.5 \mathrm{~g}\right.$ in $200 \mathrm{~mL} \mathrm{H}_{2} \mathrm{O}, 5 \mathrm{~g}$ $\mathrm{NaHCO}_{3}$ ) for detection. Visible light reactions were irradiated with blue LED (465 nm, $5 \mathrm{~W}$ ). Infrared spectra (IR) were measured on a DIGILAB 3100 FT-IR EXCALIBUR SERIES spectrometer and the position of the absorption bands is given in wave numbers $v\left(\mathrm{~cm}^{-1}\right) .{ }^{1} \mathbf{H}$ NMR (300 MHz, $400 \mathrm{MHz}$ and $600 \mathrm{MHz}),{ }^{13} \mathbf{C}\left\{{ }^{1} \mathbf{H}\right\}$ NMR $(75 \mathrm{MHz}, 100 \mathrm{MHz}$ and $151 \mathrm{MHz})$ and ${ }^{11} \mathrm{~B}$ NMR (96 MHz, $128 \mathrm{MHz}$ and $192 \mathrm{MHz}$ ) spectra were measured on a BRUKER DPX 300, BRUKER AV 300, BRUKER AV 400 or an AGILENT DD2 600 spectrometer. The multiplicity of all signals was described as s (singlet), d (doublet), $t$ (triplet), q (quartet), $p$ (pentet), h (hextet), hept (heptet) and m (multiplet). Chemical shifts ( $\delta$ in ppm) were referenced on the residual peak of $\mathrm{CDCl}_{3}\left({ }^{1} \mathrm{H} \mathrm{NMR}: \delta=7.26 ;{ }^{13} \mathrm{C} \mathrm{NMR}: \delta=77.0\right), \mathrm{C}_{6} \mathrm{D}_{6}\left({ }^{1} \mathrm{H}\right.$ NMR: $\delta=7.16 ;{ }^{13} \mathrm{C}$ NMR: $\left.\delta=128.0\right), \mathrm{CD}_{2} \mathrm{Cl}_{2}\left({ }^{1} \mathrm{H}\right.$ NMR: $\left.\delta=5.32 ;{ }^{13} \mathrm{C} \mathrm{NMR}: \delta=53.5\right)$. HRMS ESI (m/z) measurements were performed on a BRUKER MICROTOF. HRMS APCI $(\mathrm{m} / \mathrm{z})$ measurements were performed on a THERMO FISHER SCIENTIFIC ORBITRAP LTQ XL.

Chiral HPLC was performed on a HEWLETT PACKARD Series 1100 HPLC using UV detection (210 nm, $230 \mathrm{~nm}, 250 \mathrm{~nm}, 260 \mathrm{~nm}, 280 \mathrm{~nm})$. Separation was performed using CHIRALPAK®AD-H (4.6 x $250 \mathrm{~nm}$ x $5 \mu \mathrm{m}$, DAICEL CHEMICAL INDUSTRIES, LTD.) or CHIRALCEL®OJ-RH (4.5 x $150 \mathrm{~nm}$ x $5 \mu \mathrm{m}$, DAICEL CHEMICAL INDUSTRIES, LTD.) columns. Optical rotations were measured on a JASCO P-2000 polarimeter. The correct assignment of 
the diastereomers was supported by $2 \mathrm{D}-\mathrm{NMR}$ data. The ${ }^{13} \mathrm{C}$-NMR-signals for the carbons next to boron were not observed. 


\section{Experimental Procedures}

\subsection{4,4-Diethoxybutyronitrile}

1-Chloro-3,3-diethoxypropane (97.9 g, $59.0 \mathrm{mmol}, 1.0$ equiv.) was dissolved

$\mathrm{NC}$ ○ in dimethylsulfoxide $(140 \mathrm{~mL})$ and the solution slowly added to a suspension of sodium cyanide ( $31.9 \mathrm{~g}, 65.0 \mathrm{mmol}, 1.1$ equiv.) in dimethylsulfoxide (140 mL). The mixture was heated to $80{ }^{\circ} \mathrm{C}$ and stirred for $24 \mathrm{~h}$, before it was poured into water $(200 \mathrm{~mL})$. The solution was extracted with $\mathrm{Et}_{2} \mathrm{O}(3 \times 200 \mathrm{~mL})$, the combined organic phases were dried over $\mathrm{MgSO}_{4}$ and the solvent removed in vacuo. The residue was purified via fractional distillation and the product obtained as a colorless liquid. (79.5 g, $50.6 \mathrm{mmol}, 86 \%$ ) ${ }^{1} \mathbf{H}$ NMR (CDCl, 300 MHz) $\delta 4.57(\mathrm{t}, J=5.3 \mathrm{~Hz}, 1 \mathrm{H}), 3.72-3.62(\mathrm{~m}, 2 \mathrm{H}), 3.56-3.46(\mathrm{~m}$, 2H), $2.42(\mathrm{t}, J=7.3 \mathrm{~Hz}, 2 \mathrm{H}), 1.96-1.90(\mathrm{~m}, 2 \mathrm{H}), 1.21(\mathrm{t}, J=7.0 \mathrm{~Hz}, 6 \mathrm{H}){ }^{{ }^{13} \mathbf{C}}\left\{{ }^{1} \mathbf{H}\right\} \mathbf{N M R}$ $\left(\mathbf{C D C l}_{3}, 75 \mathrm{MHz}\right) \delta$ 119.6, 101.0, 62.5, 29.8, 15.4, 12.6.

The analytical data are in accordance to those described in the literature. ${ }^{1}$

\subsection{6,7-Dimethoxy-2-naphthonitrile (8)}

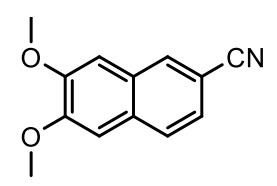

In analogy to a procedure ${ }^{2}$ by YAMADA et al., diisopropylamine $(11.2 \mathrm{~mL}$, $80.0 \mathrm{mmol}, 1.2$ equiv.) was dissolved in THF (60 mL) and cooled to $-78{ }^{\circ} \mathrm{C} . n$-Butyllithium (1.6 $\mathrm{M}$ in hexanes, $46 \mathrm{~mL}, 73 \mathrm{mmol}, 1.1$ equiv.) was slowly added to the solution via a syringe pump and the reaction was stirred for $1 \mathrm{~h}$ before it was allowed to warm to $0{ }^{\circ} \mathrm{C}$. The solution was again cooled to $-78{ }^{\circ} \mathrm{C}$ and 4,4 diethoxybutyronitrile (11.4 g, $73.0 \mathrm{mmol}, 1.1$ equiv.), dissolved in THF (17.5 mL), was added dropwise. After $1 \mathrm{~h}$ veratraldehyde $(11.1 \mathrm{~g}, 66.5 \mathrm{mmol})$, dissolved in THF $(17.5 \mathrm{~mL})$, was added and the solution slowly warmed to rt. The volatiles were removed in vacuo and water (200 $\mathrm{mL})$ was added to the residue. The mixture was extracted with ethyl acetate $(3 \times 200 \mathrm{~mL})$ and the combined organic phases were dried over $\mathrm{MgSO}_{4}$. After removal of the solvent in vacuo, the crude product was dissolved in $\mathrm{MeOH}(100 \mathrm{~mL})$ and sulfuric acid $(20 \%, 700 \mathrm{~mL})$. It was refluxed and stirred for $4 \mathrm{~h}$. The solution was cooled to rt., extracted with ethyl acetate $(3 \times 200 \mathrm{~mL})$, the combined organic phases dried over $\mathrm{MgSO}_{4}$ and the solvent removed in vacuo. After recrystallization, the product was obtained as a pale-yellow solid (11.5 g, $53.9 \mathrm{mmol}, 81 \%)$.

${ }^{1} \mathbf{H}$ NMR $\left(\mathbf{C D C l}_{3}, 300 \mathrm{MHz}\right) \delta 8.03(\mathrm{~d}, J=1.7 \mathrm{~Hz}, 1 \mathrm{H}), 7.73(\mathrm{~d}, J=8.4 \mathrm{~Hz}, 1 \mathrm{H}), 7.47(\mathrm{dd}, J$ $=8.4,1.7 \mathrm{~Hz}, 1 \mathrm{H}), 7.14-7.12(\mathrm{~m}, 2 \mathrm{H}), 4.03(\mathrm{~s}, 3 \mathrm{H}), 4.02(\mathrm{~s}, 3 \mathrm{H}) .{ }^{\mathbf{1}} \mathbf{C}\left\{{ }^{1} \mathbf{H}\right\} \mathbf{~ N M R}\left(\mathbf{C D C l}_{\mathbf{3}}\right.$, 
75 MHz) $\delta 152.1,150.9,132.1,131.4,128.5,127.4,125.4,119.8,107.5,106.6,106.4,56.2$, 56.2 .

The analytical data are in accordance to those described in the literature. ${ }^{3}$

\section{3. (S)-2-(6,7-Dimethoxynaphthaline-2-yl)-4-isopropyl-4,5- dihydrooxazole (6)}

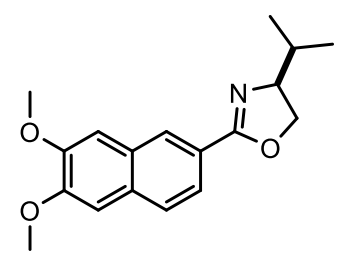

In analogy to a patented procedure ${ }^{4}$ by THUY et al., nitrile $8(213 \mathrm{mg}$, $1.00 \mathrm{mmol}, 1.0$ equiv.) was suspended in dry ethanol $(10 \mathrm{~mL})$ and cooled to $0{ }^{\circ} \mathrm{C}$. At that temperature $\mathrm{HCl}$ gas was bubbled through the suspension for $2 \mathrm{~h}$ and the mixture was further stirred overnight. After removal of the volatiles in vacuo, the thus formed imidate was resuspended in $\mathrm{CH}_{2} \mathrm{Cl}_{2}$ (10 mL) and (S)-valinol (206 mg, $223 \mu \mathrm{L}, 2.0$ equiv.) was added. The mixture was stirred for $24 \mathrm{~h}$ at $40{ }^{\circ} \mathrm{C}$, until monitoring by TLC showed that no more imidate was present. The solvent was removed in vacuo and the residue separated via FC to obtain the product as a colorless solid (246 mg, $0.82 \mathrm{mmol}, 82 \%$ ).

${ }^{1} \mathrm{H}$ NMR (CDCl, $\left.300 \mathrm{MHz}\right) \delta 8.30(\mathrm{~d}, J=1.7 \mathrm{~Hz}, 1 \mathrm{H}), 7.91(\mathrm{dd}, J=8.5,1.7 \mathrm{~Hz}, 1 \mathrm{H}), 7.70$ $(\mathrm{d}, J=8.5 \mathrm{~Hz}, 1 \mathrm{H}), 7.17(\mathrm{~s}, 1 \mathrm{H}), 7.13(\mathrm{~s}, 1 \mathrm{H}), 4.51-4.38(\mathrm{~m}, 1 \mathrm{H}), 4.26-4.12(\mathrm{~m}, 2 \mathrm{H}), 4.01$ (s, 3H), 3.99 (s, 3H), $1.99-1.85(\mathrm{~m}, 1 \mathrm{H}), 1.06(\mathrm{~d}, J=6.7 \mathrm{~Hz}, 3 \mathrm{H}), 0.95(\mathrm{~d}, J=6.7 \mathrm{~Hz}, 3 \mathrm{H})$. ${ }^{13} \mathbf{C}\left\{{ }^{1} \mathrm{H}\right\}$ NMR $\left(\mathbf{C D C l}_{3}, 75 \mathrm{MHz}\right) \delta 164.0,150.9,150.0,131.0,128.6,127.3,126.5,123.6$, $123.5,107.2,106.3,72.6,70.3,56.1,56.0,33.0,19.1,18.2$.

HRMS (ESI): Calculated for $\mathrm{C}_{18} \mathrm{H}_{21} \mathrm{O}_{3} \mathrm{NH}^{+}: m / z=300.1594\left([\mathrm{M}+\mathrm{H}]^{+}\right)$, found: 300.1608 . IR (neat): 3007, 2969, 2958, 2932, 2893, 2867, 1643, 1625, 1603, 1513, 1490, 1466, 1452, $1435,1416,1380,1366,1352,1323,1259,1244,1199,1180,1161,1125,1069,1044,1032$, $1006,975,957,916,865,749,725$.

\section{4. (S)-2-((1S,2S)-1-(Dimethyl(phenyl)silyl)-6,7-dimethoxy-2-methyl-1,2-} dihydronaphthalene-2-yl)-4-isopropyl-4,5-dihydrooxazole (9)

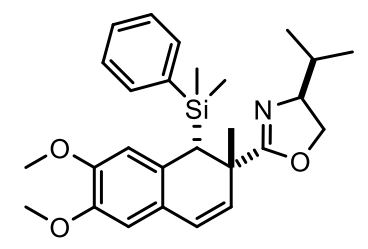

\section{Preparation of a dimethylphenylsilyllithium solution in THF:}

Freshly cut lithium wire (140 mg, $20.0 \mathrm{mmol}, 6.0$ equiv.) was suspended in THF $(10 \mathrm{~mL})$ followed by addition of dimethylphenylchlorosilane (567 mg, $3.34 \mathrm{mmol}, 1.0$ equiv.) at rt. The mixture was stirred for $6 \mathrm{~h}$ and titrated with $N$-benzylbenzamide in THF at $-20^{\circ} \mathrm{C}$ before use. Oxazoline 6 (200 mg, $660 \mu \mathrm{mol}, 1.0$ equiv.) was dissolved in THF (10 mL) and cooled to $-20^{\circ} \mathrm{C}$. While vigorous stirring dimethylphenylsilyllithium $(0.5 \mathrm{M}, 5.28 \mathrm{~mL}, 4.0$ equiv.) 
was added via syringe pump over the course of $15 \mathrm{~min}$ and the resulting red solution was stirred for $12 \mathrm{~h}$ before the addition of dimethylsulfate ( $416 \mathrm{mg}, 3.30 \mathrm{mmol}, 5.0$ equiv.). The mixture was warmed to $\mathrm{rt}$ and stirred for another $6 \mathrm{~h}$, followed by the addition of sat. aq. $\mathrm{NH}_{4} \mathrm{Cl}(10 \mathrm{~mL})$ and extraction with $\mathrm{CH}_{2} \mathrm{Cl}_{2}(3 \times 30 \mathrm{~mL})$. The combined organic phases were dried over $\mathrm{Na}_{2} \mathrm{SO}_{4}$ and the product was separated via $\mathrm{FC}$ (pentane/ $\mathrm{CH}_{2} \mathrm{Cl}_{2} /$ Acetone, 90:5:5) to yield the product as a colorless oil $(0.24 \mathrm{~g}, 0.53 \mathrm{mmol}, 80 \%)$.

${ }^{1} \mathbf{H}$ NMR (C6 $\mathbf{C}_{6}, 500$ MHz) $\delta 7.49$ - 7.45 (m, 2H), 7.17 - 7.14 (m, 3H), 6.69 (dd, $J=9.6,1.2$ $\mathrm{Hz}, 1 \mathrm{H}), 6.47$ (s, 1H), 6.27 (d, $J=9.6 \mathrm{~Hz}, 1 \mathrm{H}), 6.13(\mathrm{~s}, 1 \mathrm{H}), 3.82-3.78(\mathrm{~m}, 1 \mathrm{H}), 3.55-3.45$ (m, 2H), $3.44(\mathrm{~s}, 3 \mathrm{H}), 3.29(\mathrm{~s}, 3 \mathrm{H}), 3.09-3.06(\mathrm{~m}, 1 \mathrm{H}), 1.59-1.52(\mathrm{~m}, 4 \mathrm{H}), 1.10(\mathrm{~d}, J=6.6$ $\mathrm{Hz}, 3 \mathrm{H}), 0.73$ (d, J = 6.7 Hz, 3H), 0.40 (s, 3H), 0.19 (s, 3H) ${ }^{13} \mathbf{C}\left\{{ }^{1} \mathbf{H}\right\}$ NMR (C6 $\mathbf{~ D 6}, 125$ MHz): $\delta 171.1,149.2,147.9,140.1,134.7,131.4,129.4,128.8,127.6,127.2,125.7,113.5,111.3$, 73.3, 70.7, 55.9, 55.4, 40.7, 40.5, 33.6, 27.1, 19.8, 19.2, -0.5, -1.8.

HRMS (ESI): Calculated for $\mathrm{C}_{27} \mathrm{H}_{35} \mathrm{O}_{3} \mathrm{NSiH}^{+}: \mathrm{m} / z=450.2459\left([\mathrm{M}+\mathrm{H}]^{+}\right)$, found: 450.2470 . $[\boldsymbol{\alpha}]_{\boldsymbol{D}}^{\mathbf{2 0}}=+169.6^{\circ}$ in $\mathrm{CHCl}_{3}$ (Conc. $11.1 \mathrm{mg} / \mathrm{ml}$ ).

IR (neat): 3068, 3046, 2958, 2931, 2871, 2834, 2361, 1662, 1604, 1507, 1465, 1453, 1427 , 1411, 1360, 1336, 1305, 1282, 1266, 1248, 1231, 1200, 1133, 1111, 1082, 1030, 1000, 980, 933, 888, 865, 837, 811, 788, 734, 701, 638.

2.5. (S)-6,7-Dimethoxy-2-methyl-1,2-dihydronaphthalene-2-carboxylic acid (5)<smiles>C=CC1(C(=O)O)Cc2cc(OC)c(OC)cc2C1</smiles>

Oxazoline 9 (1.00 g, $2.20 \mathrm{mmol}, 1.0$ equiv.) was dissolved in 1,4-dioxane $(11 \mathrm{~mL})$ followed by the addition of aqueous $\mathrm{HCl}(6 \mathrm{M}, 11 \mathrm{~mL})$. The mixture was heated to $100{ }^{\circ} \mathrm{C}$ for $12 \mathrm{~h}$ and after cooling to rt. extracted with $\mathrm{CH}_{2} \mathrm{Cl}_{2}$ (3x100 mL). The combined organic phases were dried over $\mathrm{Na}_{2} \mathrm{SO}_{4}$ and the solvent removed in vacuo. After separation by $\mathrm{FC}\left(\mathrm{MeOH} / \mathrm{AcOH} / \mathrm{CH}_{2} \mathrm{Cl}_{2}, 2: 1: 97\right)$ the product was obtained as a colorless solid. $(375 \mathrm{mg}, 1.51 \mathrm{mmol}, 68 \%$, ee $=99 \%)$

${ }^{1} \mathbf{H}$ NMR ( $\left.\mathbf{C D}_{2} \mathbf{C l}_{2}, 500 \mathrm{MHz}\right) \delta 6.69(\mathrm{~s}, 1 \mathrm{H}), 6.64(\mathrm{~s}, 1 \mathrm{H}), 6.42(\mathrm{~d}, J=9.6 \mathrm{~Hz}, 1 \mathrm{H}), 5.91$ (d, $J$ $=9.6 \mathrm{~Hz}, 1 \mathrm{H}), 3.82(\mathrm{~s}, 3 \mathrm{H}), 3.80(\mathrm{~s}, 3 \mathrm{H}), 3.22(\mathrm{~d}, J=15.7 \mathrm{~Hz}, 1 \mathrm{H}), 2.75(\mathrm{~d}, J=15.7 \mathrm{~Hz}, 1 \mathrm{H})$, 1.32 (s, 3H). ${ }^{13} \mathbf{C}\left\{{ }^{1} \mathbf{H}\right\}$ NMR ( $\left.\mathbf{C D}_{2} \mathbf{C l}_{2}, \mathbf{1 2 5} \mathbf{M H z}\right): \delta 181.7,149.2,148.4,129.7,127.7,126.2$, 125.5, 112.6, 110.8, 56.5, 56.4, 43.7, 37.8, 24.1.

HRMS (ESI): Calculated for $\mathrm{C}_{14} \mathrm{H}_{16} \mathrm{O}_{4} \mathrm{Na}^{+}: m / z=271.0941\left([\mathrm{M}+\mathrm{Na}]^{+}\right)$, found: 271.0949 . $[\boldsymbol{\alpha}]_{\boldsymbol{D}}^{\mathbf{2 0}}=-89.1^{\circ}$ in $\mathrm{CDCl}_{3}$ (Conc. $10 \mathrm{mg} / \mathrm{ml}$ ).

IR (neat): 2933, 2872, 2836, 1699, 1606, 1574, 1511, 1455, 1413, 1384, 1337, 1310, 1247 , 1232, 1148, 1116, 1098, 1031, 1000, 862, 823, 757, 662. 
MP: $121-124{ }^{\circ} \mathrm{C}$

\section{6. (S)-Methyl 6,7-Dimethoxy-2-methyl-1,2-dihydronaphthalene-2- carboxylate (5-OMe)}<smiles>COC(=O)C1(C)Cc2ccc(OC)c(OC)c2C1</smiles>

Carboxylic acid 5 (0.10 g, $0.40 \mathrm{mmol}, 1.0$ equiv.) was dissolved in DMF $(10 \mathrm{~mL})$ and potassium carbonate $(1.0 \mathrm{~g}, 7.2 \mathrm{mmol}, 18$ equiv.) was added.

After the addition of iodomethane $(2.3 \mathrm{~g}, 1.0 \mathrm{~mL}, 16 \mathrm{mmol}, 40$ equiv.) the suspension was stirred for $24 \mathrm{~h}$ at rt. Water $(20 \mathrm{~mL})$ was added, the solution was extracted with $\mathrm{Et}_{2} \mathrm{O}(3 \times 50 \mathrm{~mL})$ and the combined organic phases dried over $\mathrm{MgSO}_{4}$. The solvent was removed in vacuo and after separation via FC on silica, the product was obtained as a colorless solid (92 mg, $0.35 \mathrm{mmol}, 88 \%$, ee $=99 \%$ ).

${ }^{1} \mathrm{H}$ NMR (CDCl, $\left.600 \mathrm{MHz}\right) \delta 6.68(\mathrm{~d}, J=0.9 \mathrm{~Hz}, 1 \mathrm{H}), 6.61(\mathrm{~s}, 1 \mathrm{H}), 6.38(\mathrm{~d}, J=9.6 \mathrm{~Hz}$, $1 \mathrm{H}), 5.93(\mathrm{~d}, J=9.6 \mathrm{~Hz}, 1 \mathrm{H}), 3.88(\mathrm{~s}, 3 \mathrm{H}), 3.85$ (s, 3H), 3.70 (s, 3H), 3.25 (d, $J=15.6 \mathrm{~Hz}$, 1H), $2.74(\mathrm{~d}, J=15.6 \mathrm{~Hz}, 1 \mathrm{H}), 1.29(\mathrm{~s}, 3 \mathrm{H}){ }^{\mathbf{1}} \mathbf{C}\left\{{ }^{\mathbf{1}} \mathbf{H}\right\} \mathbf{N M R}\left(\mathbf{C D C l}_{3}, \mathbf{1 5 0} \mathbf{M H z}\right) \delta 177.0,148.4$, $147.7,129.8,126.9,125.9,125.2,111.9,110.1,56.1,56.1,52.3,43.6,37.6,23.9$.

HRMS (ESI): Calculated for $\mathrm{C}_{15} \mathrm{H}_{18} \mathrm{O}_{4} \mathrm{Na}^{+}: \mathrm{m} / z=285.1097\left([\mathrm{M}+\mathrm{Na}]^{+}\right)$, found: 285.1107.

IR (neat): 2962, 2862, 1720, 1603, 1573, 1519, 1472, 1450, 1435, 1412, 1382, 1358, 1342, $1315,1262,1242,1230,1211,1192,1147,1116,1105,1070,1034,1001,965,929,893,860$, $843,823,772,757,675,654,608$.

\subsection{Arylation, borylation, homologation sequence: Synthesis of boronic ester 17}

Table 1. Optimization studies on the decarboxylative $\gamma$-veratrylation of 5 .<smiles>COc1cc2c(cc1OC)CC(C)(C(=O)O)C=C2</smiles>

5

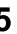

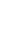

$$
\begin{aligned}
& X=I, 11 \\
& X=B r, 12
\end{aligned}
$$

$\mathrm{Pd}(\mathrm{dba})_{2}(10 \mathrm{~mol} \%)$, $\mathrm{Cs}_{2} \mathrm{CO}_{3}$ (1.3 equiv.)

PhMe (0.3 M), $110^{\circ} \mathrm{C}, 18 \mathrm{~h}$

\begin{tabular}{|c|c|c|c|}
\hline Entry & $\mathrm{X}$ & Temperature & $13 / 14^{\mathrm{a}}$ \\
\hline 1 & $\mathrm{I}$ & $110^{\circ} \mathrm{C}$ & $54 \%$ \\
\hline 2 & $\mathrm{I}$ & $90^{\circ} \mathrm{C}$ & $74 \%$ \\
\hline 3 & $\mathrm{Br}$ & $110^{\circ} \mathrm{C}$ & $99 \%$ \\
\hline
\end{tabular}<smiles>[R]C1=C(C)Cc2cc(OC)c(OC)cc2[C@H]1c1ccc(OC)c(OC)c1</smiles>

$\mathrm{R}=\mathrm{H}, \mathbf{4}$

$\mathrm{R}=$ 4-veratryl, 14

a) Ratio determined by GC-FID. 


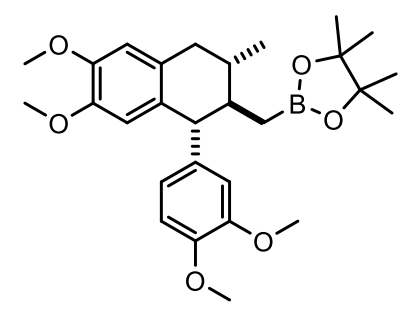

Optimized conditions: Carboxylic acid 5 (12.5 mg, $50.3 \mu \mathrm{mol}$, 1.0 equiv.), 4-veratrylbromide ( $12 \mathrm{mg}, 8.0 \mu \mathrm{L}, 55.3 \mu \mathrm{mol}$, 1.1 equiv.), $\mathrm{Cs}_{2} \mathrm{CO}_{3}\left(19.5 \mathrm{mg}, 60.4 \mu \mathrm{mol}, 1.2\right.$ equiv.) and $\mathrm{Pd}(\mathrm{dba})_{2}$ (2.8 mg, $4.8 \mu \mathrm{mol}, 9.6 \mathrm{~mol} \%)$ were suspended in dry toluene $(1 \mathrm{~mL})$ and degassed via three freeze-pump-thaw degassing cycles. The mixture was warmed to $110{ }^{\circ} \mathrm{C}$ and stirred for $18 \mathrm{~h}$ followed by the addition of triethylsilane ( $24 \mu \mathrm{L}, 0.15 \mathrm{mmol}, 3.0$ equiv.) at rt. The resulting solution was allowed to settle and filtered into a solution of trichloroborane in $\mathrm{CH}_{2} \mathrm{Cl}_{2}(1 \mathrm{M}, 0.15 \mathrm{mmol}, 0.15 \mathrm{~mL}, 3.0$ equiv. $)$ at $0{ }^{\circ} \mathrm{C}$ via a cannula. The residue left in the reaction flask was washed with $\mathrm{CH}_{2} \mathrm{Cl}_{2}(1 \mathrm{~mL})$ and was likewise added to the trichloroborane. After stirring for 10 minutes the solution was warmed to rt. and stirred for another $2 \mathrm{~h}$ before 2,2-dimethylpropane-1,3-diol (16 mg, $0.15 \mathrm{mmol}$, 3.0 equiv.) was added to the flask in one portion. The solvent was removed in vacuo and the resulting residue was taken up into THF $(1.5 \mathrm{~mL})$ before the addition of chloro iodomethane (88 mg, $0.50 \mathrm{mmol}, 37 \mu \mathrm{L}, 10$ equiv.). The clear solution was cooled to $-78{ }^{\circ} \mathrm{C}$ and $n$ butyllithium in hexanes ( $1.6 \mathrm{M}, 0.40 \mathrm{mmol}, 0.25 \mathrm{~mL}, 8.0$ equiv.) was added via syringe pump in the course of $15 \mathrm{~min}$. The mixture was further stirred for $20 \mathrm{~min}$ at the same temperature before it was warmed to rt. For the transesterification, aqueous sodium hydroxide $(0.2 \mathrm{M}$, $0.5 \mathrm{~mL}$ ) was poured directly into the reaction mixture followed by the addition of pinacol (30 mg, $0.25 \mathrm{mmol}, 5.0$ equiv) and stirring for $24 \mathrm{~h}$. The volatiles were removed in vacuo, the residue taken up on $\mathrm{C}_{18}$-silica and separated using a BÜCHI REVELERIS ${ }^{\circledR}$ automated FC with a $4 \mathrm{~g} \mathrm{C}_{18}$-silica column and $\mathrm{H}_{2} \mathrm{O}: \mathrm{MeCN}$ as the eluent. The product was isolated as a colorless oil with a dr. of 5:1 (12.3 mg, $25.5 \mu \mathrm{mol}, 51 \%)$.

${ }^{1} \mathbf{H}$ NMR (C6 $\left.\mathbf{D}_{6}, 600 \mathrm{MHz}\right)$ (major): $\delta 6.97(\mathrm{dd}, J=8.1,2.0 \mathrm{~Hz}, 1 \mathrm{H}), 6.92(\mathrm{~d}, J=2.0 \mathrm{~Hz}$, $1 \mathrm{H}), 6.68(\mathrm{~d}, J=8.1 \mathrm{~Hz}, 1 \mathrm{H}), 6.56(\mathrm{~s}, 1 \mathrm{H}), 6.53(\mathrm{~s}, 1 \mathrm{H}), 4.15(\mathrm{~d}, J=10.9 \mathrm{~Hz}, 1 \mathrm{H}), 3.49$ (s, $3 \mathrm{H}), 3.43(\mathrm{~s}, 3 \mathrm{H}), 3.43(\mathrm{~s}, 3 \mathrm{H}), 3.27(\mathrm{~s}, 3 \mathrm{H}), 2.75(\mathrm{dd}, J=16.0,4.9 \mathrm{~Hz}, 1 \mathrm{H}), 2.69$ (dd, $J=$ 15.6, $11.1 \mathrm{~Hz}, 1 \mathrm{H}), 2.18-2.09(\mathrm{~m}, 1 \mathrm{H}), 2.09-2.01(\mathrm{~m}, 1 \mathrm{H}), 1.28(\mathrm{dd}, J=15.9,4.4 \mathrm{~Hz}, 1 \mathrm{H})$, $1.25(\mathrm{~d}, J=6.5 \mathrm{~Hz}, 3 \mathrm{H}), 1.12(\mathrm{dd}, J=15.9,4.4 \mathrm{~Hz}, 1 \mathrm{H}) 1.05(\mathrm{~s}, 6 \mathrm{H}), 1.04(\mathrm{~s}, 6 \mathrm{H})$.

${ }^{1} \mathbf{H}$ NMR (C6 $\left.\mathbf{D}_{6}, 600 \mathrm{MHz}\right)$ (minor): $\delta 6.95(\mathrm{~d}, J=2.1 \mathrm{~Hz}, 1 \mathrm{H}), 6.76(\mathrm{dd}, J=8.2,2.1 \mathrm{~Hz}$, $1 \mathrm{H}), 6.64(\mathrm{~d}, J=8.3 \mathrm{~Hz}, 1 \mathrm{H}), 6.62(\mathrm{~s}, 1 \mathrm{H}), 6.57(\mathrm{~s}, 1 \mathrm{H}), 4.36(\mathrm{~d}, J=4.9 \mathrm{~Hz}, 1 \mathrm{H}), 3.55(\mathrm{~s}, 3 \mathrm{H})$, $3.51(\mathrm{~s}, 3 \mathrm{H}), 3.42(\mathrm{~s}, 3 \mathrm{H}), 3.25(\mathrm{~s}, 3 \mathrm{H}), 2.93(\mathrm{dd}, J=16.7,5.5 \mathrm{~Hz}, 1 \mathrm{H}), 2.48(\mathrm{dd}, J=16.7$, $10.0 \mathrm{~Hz}, 1 \mathrm{H}), 2.41-2.34(\mathrm{~m}, 1 \mathrm{H}), 2.19-2.09(\mathrm{~m}, 1 \mathrm{H}), 1.11(\mathrm{~s}, 6 \mathrm{H}), 1.11(\mathrm{~s}, 6 \mathrm{H}), 1.05-1.01$ $(\mathrm{m}, 4 \mathrm{H}), 0.95-0.90(\mathrm{~m}, 1 \mathrm{H})$. 
${ }^{13} \mathbf{C}\left\{{ }^{1} \mathbf{H}\right\}$ NMR (C6 $\mathbf{6} 6,150$ MHz) (major): $\delta$ 149.9, 148.4, 148.3, 148.2, 139.4, 133.0, 129.1, $122.1,114.3,113.4,112.0,111.8,82.4,55.4,55.3,55.2,55.2,53.2,46.0,39.4,34.9$, 24.6, 24.5, 20.4 .

${ }^{13} \mathbf{C}\left\{{ }^{1} \mathbf{H}\right\}$ NMR (C6. 6 6, 150 MHz) (minor): $\delta$ 149.1, 148.5, 148.4, 148.3, 136.5, 132.3, 128.5, 125.5, 123.1, 115.5, 113.7, 112.0, 111.7, 82.5, 55.4, 55.3, 55.1, 49.9, 41.6, 38.0, 30.1, 29.4, $24.8,24.5,19.8$.

HRMS (ESI): Calculated for $\mathrm{C}_{28} \mathrm{H}_{39} \mathrm{O}_{6} \mathrm{BNa}^{+}: m / z=505.2737\left([\mathrm{M}+\mathrm{Na}]^{+}\right)$, found: 505.2732 . IR (neat): 2935, 2834, 1608, 1590, 1514, 1465, 1417, 1371, 1318, 1248, 1219, 1144, 1113, 1090, 1030, 1004, 968, 892, 867, 848, 811.

\subsection{Galbulin (1)}<smiles>COc1ccc([C@@H]2c3cc(OC)c(OC)cc3C[C@@H](C)[C@H]2C)cc1OC</smiles>

Boronic ester 17 (15.5 mg, $32.0 \mu \mathrm{mol}, 1.0$ equiv., $\mathrm{dr}=5: 1)$ was dissolved in THF $(1.5 \mathrm{~mL})$ and cooled to $-78{ }^{\circ} \mathrm{C}$. Phenyllithium in di- $n$-butylether (1.9 M, $19 \mu \mathrm{L}, 1.1$ equiv.) was added and the solution warmed to rt. afterwards. After stirring for $1 \mathrm{~h}$, the volatiles were removed in vacuo and the resulting residue was dissolved in $\mathrm{MeOH}(0.75 \mathrm{~mL})$ and acetone (0.75 mL). Thiophenol (3.8 mg, $3.5 \mu \mathrm{L}, 1.1$ equiv.) and $\operatorname{Ir}(\mathrm{dFFppy})_{2}(\mathrm{dtb}-\mathrm{bpy}) \mathrm{PF}_{6}(0.71 \mathrm{mg}$, $2 \mathrm{~mol} \%$ ) were added and the mixture degassed via three freeze-pump-thaw degassing cycles and irradiated with a LED ( $5 \mathrm{~W}, 460 \mathrm{~nm})$ for $18 \mathrm{~h}$ at rt. After separation via FC (toluene/pentane/ethylacetate, 10:10:1) galbulin was isolated as a colorless solid with a dr of 5:1. (7.6 mg, $21 \mu \mathrm{mol}, 80 \%$, ee $>99 \%$ ) After preparative HPLC, the product was isolated as single diastereomer for characterization.

${ }^{1}$ H NMR (C6D6, 500 MHz) $\delta 6.76(\mathrm{dd}, J=8.1,2.0 \mathrm{~Hz}, 1 \mathrm{H}), 6.69(\mathrm{~d}, J=2.0 \mathrm{~Hz}, 1 \mathrm{H}), 6.66(\mathrm{~d}$, $J=8.1 \mathrm{~Hz}, 1 \mathrm{H}), 6.53(\mathrm{~s}, 1 \mathrm{H}), 6.51(\mathrm{~s}, 1 \mathrm{H}), 3.53-3.49(\mathrm{~m}, 4 \mathrm{H}), 3.44(\mathrm{~s}, 3 \mathrm{H}), 3.35(\mathrm{~s}, 3 \mathrm{H})$, $3.29(\mathrm{~s}, 3 \mathrm{H}), 2.74-2.58(\mathrm{~m}, 2 \mathrm{H}), 1.69-1.60(\mathrm{~m}, 2 \mathrm{H}), 1.05(\mathrm{~d}, J=6.0 \mathrm{~Hz}, 3 \mathrm{H}), 0.97(\mathrm{~d}, J=$ $5.8 \mathrm{~Hz}, 3 \mathrm{H}){ }^{13} \mathbf{C}\left\{{ }^{1} \mathbf{H}\right\}$ NMR (C6. $\left.\mathbf{~} 6 \mathbf{1 2 5} \mathbf{M H z}\right): \delta 150.1,148.5,148.4,148.3,139.2,132.6$, 129.0, 122.0, 114.2, 113.0, 111.9, 111.8, 55.4, 55.4, 55.2, 55.2, 54.5, 44.2, 39.2, 35.7, 19.9, 17.1.

HRMS (ESI): Calculated for $\mathrm{C}_{22} \mathrm{H}_{28} \mathrm{O}_{4} \mathrm{Na}^{+}: m / z=379.1880\left([\mathrm{M}+\mathrm{Na}]^{+}\right)$, found: 379.1869 .

IR (neat): 2929, 2831, 1609, 1590, 1516, 1465, 1419, 1354, 1295, 1248, 1217, 1158, 1139, 1109, 1072, 1030, 1006, 899, 860, 805, 766.

MP: $131-132{ }^{\circ} \mathrm{C}\left(\mathrm{H}_{2} \mathrm{O}\right)$.

$[\alpha]_{D}^{23}=+8^{\circ}$ in $\mathrm{CHCl}_{3}$ (Conc. $0.6 \mathrm{mg} / \mathrm{ml}$ ).

The analytical data are in accordance to the literature. ${ }^{5}$ 


\subsection{Rac-(S)-4-(tert-butyl)-2-(naphthalen-2-yl)-4,5-dihydrooxazole (22)}

2-Naphthoyl chloride (21) (5.00 g, $26.2 \mathrm{mmol}, 1.0$ equiv.) was dissolved in dichloromethane

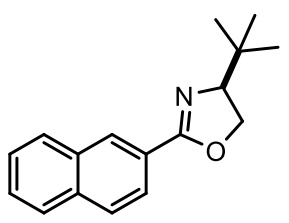

$(50 \mathrm{~mL})$ followed by the addition of rac-tert leucinol $(4.61 \mathrm{~g}, 39.3 \mathrm{mmol}$,

1.5 equiv.). Triethylamine (5.00 g, $50.0 \mathrm{mmol}, 1.9$ equiv.) was added slowly whilst the reaction mixture was stirred at rt. After the mixture was stirred overnight it was washed with water using a separation funnel. The organic phase was collected and dried over magnesium sulfate. The solution was transferred back into a reaction vessel and the solvent was removed in vacuo. A solid residue remained in the flask which was dissolved in thionyl chloride $(30 \mathrm{~mL})$ and stirred for another $3 \mathrm{~h}$. The remaining thionyl chloride was removed in vacuo and the residue was recrystallized from toluene and $n$-heptane to yield the product as a colorless fluffy solid. (5.32 g, $15.1 \mathrm{mmol}$, $58 \%)$

${ }^{1} \mathbf{H}$ NMR (CDCl, $\left.300 \mathrm{MHz}\right) \delta 8.45(\mathrm{t}, J=1.1 \mathrm{~Hz}, 1 \mathrm{H}), 8.07(\mathrm{dd}, J=8.6,1.7 \mathrm{~Hz}, 1 \mathrm{H}), 7.96-$ $7.82(\mathrm{~m}, 3 \mathrm{H}), 7.60-7.47(\mathrm{~m}, 2 \mathrm{H}), 4.41(\mathrm{dd}, J=10.1,8.6 \mathrm{~Hz}, 1 \mathrm{H}), 4.34-4.27(\mathrm{~m}, 1 \mathrm{H}), 4.11$ $(\mathrm{dd}, J=10.1,7.6 \mathrm{~Hz}, 1 \mathrm{H}), 0.99(\mathrm{~s}, 9 \mathrm{H}) .{ }^{13} \mathbf{C}\left\{{ }^{1} \mathbf{H}\right\} \mathbf{N M R}\left(\mathbf{C D C l}_{3}, \mathbf{7 5} \mathbf{M H z}\right) \delta 163.5,134.8$, $132.8,129.0,128.7,128.1,127.9,127.5,126.6,125.4,125.2,76.5,68.9,34.3,26.1$.

The analytical data are in accordance to those described in the literature. ${ }^{6}$

\subsection{Rac-(S)-4-(tert-butyl)-2-((1S,2S)-1-(dimethyl(phenyl)silyl)-2-methyl- 1,2-dihydronaphthalen-2-yl)-4,5-dihydrooxazole (23)}

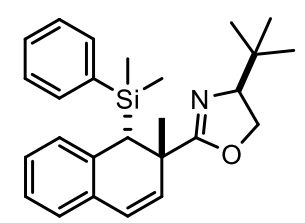

Preparation of a dimethylphenylsilyllithium solution in THF: Freshly cut lithium wire (140 mg, $20.0 \mathrm{mmol}, 6.0$ equiv.) was suspended in THF $(10 \mathrm{~mL})$ followed by addition of dimethylphenylchlorosilane $(567 \mathrm{mg}$, $3.34 \mathrm{mmol}, 1.0$ equiv.) at rt. The mixture was stirred for $6 \mathrm{~h}$ and titrated with $\mathrm{N}$-benzylbenzamide in THF at $-20{ }^{\circ} \mathrm{C}$ before use.

Oxazoline 22 (2.00 g,7.89 mmol, 1.0 equiv.) was dissolved in THF (10 mL) and cooled to $-20{ }^{\circ} \mathrm{C}$. While vigorous stirring, dimethylphenylsilyllithium $(0.9 \mathrm{M}, 18.0 \mathrm{~mL}, 2.0$ equiv. was added via syringe pump over the course of $15 \mathrm{~min}$ and the resulting red solution was stirred for $12 \mathrm{~h}$ before the addition of methyliodide ( $4.48 \mathrm{~g}, 31.6 \mathrm{mmol}, 4.0$ equiv.). The mixture was warmed to rt and stirred for another $6 \mathrm{~h}$, followed by the addition of sat. aq. $\mathrm{NH}_{4} \mathrm{Cl}(25 \mathrm{~mL})$ and extraction with $\mathrm{CH}_{2} \mathrm{Cl}_{2}(3 \times 70 \mathrm{~mL})$. The combined organic phases were dried over $\mathrm{Na}_{2} \mathrm{SO}_{4}$ and the product was separated via $\mathrm{FC}$ (pentane $/ \mathrm{CH}_{2} \mathrm{Cl}_{2} /$ Acetone, 90:5:5) to yield the product as a colorless oil $(1.94 \mathrm{~g}, 4.81 \mathrm{mmol}, 61 \%)$. 
${ }^{1} \mathbf{H}$ NMR (CDCl, $\left.300 \mathrm{MHz}\right) \delta 7.46-7.39(\mathrm{~m}, 2 \mathrm{H}), 7.34-7.23(\mathrm{~m}, 3 \mathrm{H}), 7.11-6.97(\mathrm{~m}, 3 \mathrm{H})$, $6.83-6.78(\mathrm{~m}, 1 \mathrm{H}), 6.36(\mathrm{~d}, J=9.7 \mathrm{~Hz}, 1 \mathrm{H}), 6.26(\mathrm{dd}, J=9.7,1.3 \mathrm{~Hz}, 1 \mathrm{H}), 3.83(\mathrm{dd}, J=8.6$, $8.6 \mathrm{~Hz}, 1 \mathrm{H}), 3.67$ (dd, $J=10.1,8.4 \mathrm{~Hz}, 1 \mathrm{H}), 3.44$ (dd, $J=10.0,8.8 \mathrm{~Hz}, 1 \mathrm{H}), 3.04(\mathrm{~s}, 1 \mathrm{H})$, 1.29 (s, 3H), 0.86 (s, 9H), 0.17 (s, 3H), -0.01 (s, 3H). ${ }^{13} \mathbf{C}\left\{{ }^{1} \mathbf{H}\right\} \mathbf{N M R}\left(\mathbf{C D C l}_{3}, \mathbf{7 5} \mathbf{M H z}\right) \delta$ 171.0, 139.7, 137.1, 134.3, 133.2, 132.7, 128.6, 128.5, 127.3, 127.1, 127.0, 126.1, 125.4, 75.7, $68.4,41.1,40.5,33.6,27.3,26.1,-1.2,-1.2$.

HRMS (ESI): Calculated for $\mathrm{C}_{26} \mathrm{H}_{34} \mathrm{NOSi}^{+}: \mathrm{m} / z=404.2404\left([\mathrm{M}+\mathrm{H}]^{+}\right)$, found: 404.2421 .

IR (neat): 3069, 3050, 2955, 2903, 2867, 1665, 1625, 1479, 1453, 1427, 1393, 1362, 1332 , 1303, 1245, 1194, 1140, 1114, 1082, 1062, 1026, 976, 930, 903, 873, 859, 834, 814, 777, 734, 699,640 .

\subsection{Rac-(S)-4-(tert-butyl)-2-((S)-2-methyl-1,2-dihydronaphthalen-2-yl)- 4,5-dihydrooxazole (24)}

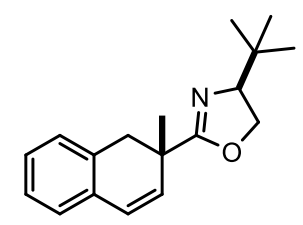

To a solution of oxazoline 23 (125 mg, $0.310 \mathrm{mmol}, 1.0$ equiv.) in THF (15 mL), tetrabutylammonium fluoride (500 mg, $1.91 \mathrm{mmol}, 6.2$ equiv.) was added at once at rt. After a reaction time of $24 \mathrm{~h}$, TLC analysis showed complete conversion of the starting material and the reaction was terminated by removal of the solvent in vacuo. The residue was separated by column chromatography to yield the product $\mathbf{2 4}$ as a colorless oil. (65.0 mg, $0.241 \mathrm{mmol}, 78 \%$ ). ${ }^{1} \mathbf{H}$ NMR $\left(\mathbf{C D C l}_{3}, \mathbf{3 0 0} \mathbf{M H z}\right) \delta 7.19-7.03(\mathrm{~m}, 4 \mathrm{H}), 6.47$ (d, $\left.J=9.7 \mathrm{~Hz}, 1 \mathrm{H}\right), 6.10$ (d, $J=9.6$ $\mathrm{Hz}, 1 \mathrm{H}), 4.20-4.05(\mathrm{~m}, 2 \mathrm{H}), 3.82(\mathrm{dd}, J=10.0,6.9 \mathrm{~Hz}, 1 \mathrm{H}), 3.29(\mathrm{~d}, J=15.6 \mathrm{~Hz}, 1 \mathrm{H}), 2.85$ $(\mathrm{d}, J=15.6 \mathrm{~Hz}, 1 \mathrm{H}), 1.29(\mathrm{~s}, 3 \mathrm{H}), 0.82(\mathrm{~s}, 9 \mathrm{H}) \cdot{ }^{\mathbf{1}} \mathbf{C}\left\{{ }^{\mathbf{1}} \mathbf{H}\right\} \mathbf{N M R}\left(\mathbf{C D C l}_{3}, \mathbf{7 5} \mathbf{M H z}\right) \delta 171.2$, 133.5, 133.2, 132.8, 128.3, 127.4, 126.8, 126. 8, 126.2, 75.5, 68.9, 38.9, 38.1, 33.9, 25.7, 23.7. HRMS (ESI): Calculated for $\mathrm{C}_{18} \mathrm{H}_{24} \mathrm{NO}^{+}: \mathrm{m} / z=270.1852\left([\mathrm{M}+\mathrm{H}]^{+}\right)$, found: 270.1851 . IR (neat): 2957, 2928, 2903, 2869, 1728, 1631, 1511, 1478, 1454, 1393, 1365, 1335, 1306, 1260, 1232, 1194, 1112, 1085, 1056, 1026, 976, 934, 915, 863, 824, 795, 780, 755, 703.

\subsection{Rac-(S)-2-methyl-1,2-dihydronaphthalene-2-carboxylic acid (20)}

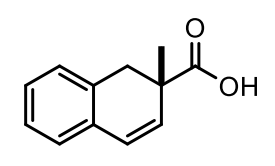

Oxazoline 24 (260 mg, $967 \mu \mathrm{mol}, 1.0$ equiv.) was dissolved in dioxane

$(10 \mathrm{~mL})$ followed by the addition of water $(2.5 \mathrm{~mL})$. Afterwards, sulfuric acid $(2.5 \mathrm{~mL}, 96 \%)$ was added dropwise and the mixture was stirred for $24 \mathrm{~h}$ whilst stirring. After the reaction had reached rt. the mixture was poured into a separation 
funnel and extracted with $\mathrm{CH}_{2} \mathrm{Cl}_{2}(3 \times 20 \mathrm{~mL})$. The combined organic phases were dried over magnesium sulfate, the solvent removed in vacuo and the product isolated as a colorless solid after $\mathrm{FC}\left(\mathrm{CH}_{2} \mathrm{Cl}_{2} / \mathrm{MeOH}, 98: 2\right)$. (116 mg, $\left.618 \mu \mathrm{mol}, 64 \%\right)$

${ }^{1} \mathbf{H}$ NMR (CDCl, 300 MHz) $\delta 7.23-6.99$ (m, 4H), 6.50 (d, $\left.J=9.6 \mathrm{~Hz}, 1 \mathrm{H}\right), 6.04$ (d, $J=9.4$ $\mathrm{Hz}, 1 \mathrm{H}), 3.32(\mathrm{~d}, J=15.8 \mathrm{~Hz}, 1 \mathrm{H}), 2.84(\mathrm{~d}, J=15.8 \mathrm{~Hz}, 1 \mathrm{H}), 1.34(\mathrm{~s}, 3 \mathrm{H}),{ }^{13} \mathbf{C}\left\{{ }^{1} \mathbf{H}\right\} \mathbf{N M R}$ $\left(\mathbf{C D C l}_{3}, \mathbf{7 5} \mathbf{M H z}\right) \delta 182.7,133.1,132.4,131.1,128.3,127.8,127.8,127.0,126.4,43.4,37.6$, 23.8.

HRMS (ESI): Calculated for $\mathrm{C}_{24} \mathrm{H}_{22} \mathrm{O}_{4} \mathrm{Na}^{-}: \mathrm{m} / z=397.1421\left([2 \mathrm{M}-2 \mathrm{H}+\mathrm{Na}]^{-}\right)$, found: 397.1418 . MP: $73-74{ }^{\circ} \mathrm{C} .($ EtOAc)

\subsection{Arylation, borylation, homologation sequence: General procedure for the synthesis of boronic esters 25 to 29}

Carboxylic acid 20 (1.0 equiv.), aryl iodide (1.1 equiv.), $\mathrm{Cs}_{2} \mathrm{CO}_{3}$ (1.2 equiv.) and $\mathrm{Pd}(\mathrm{dba})_{2}$ (20.0 mol\%) were suspended in dry toluene and degassed via three freeze-pump-thaw degassing cycles. The mixture was warmed to $110{ }^{\circ} \mathrm{C}$ and stirred for $18 \mathrm{~h}$ followed by the addition of triethylsilane (3.0 equiv.) at rt. The resulting solution was allowed to settle and filtered into a solution of trichloroborane in $\mathrm{CH}_{2} \mathrm{Cl}_{2}(1 \mathrm{M}, 3.0$ equiv. $)$ at $0{ }^{\circ} \mathrm{C}$ via a cannula. The residue left in the original reaction flask was washed with toluene and was likewise added to the trichloroborane solution. After stirring for 10 minutes the solution was warmed to rt. and stirred for another $2 \mathrm{~h}$ before 2,2-dimethylpropane-1,3-diol (5.0 equiv.) was added to the flask in one portion. The solvent was removed in vacuo and the resulting residue was taken up in THF before the addition of chloroiodomethane (10 equiv.). The clear solution was cooled to $-78{ }^{\circ} \mathrm{C}$ and $n$-butyllithium in hexanes (1.6 M, 8.0 equiv.) was added via syringe pump in the course of $15 \mathrm{~min}$. The mixture was further stirred for $20 \mathrm{~min}$ at the same temperature before it was warmed to rt. For the transesterification, aqueous sodium hydroxide $(0.2 \mathrm{M})$ was poured directly into the reaction mixture followed by the addition of pinacol (10.0 equiv) and stirring was continued for $24 \mathrm{~h}$. The volatiles were removed in vacuo, the residue taken up on $\mathrm{C}_{18^{-}}$ silica and separated using a BÜCHI REVELERIS® automated FC with a 4 g C 18 -silica column and $\mathrm{H}_{2} \mathrm{O}: \mathrm{MeCN}$ as the eluent. The products were isolated as colorless oils. 


\subsubsection{Rac-4,4,5,5-tetramethyl-2-((1R,2R,3S)-3-methyl-1-(3,4,5-}

trimethoxyphenyl)-1,2,3,4-tetrahydronaphthalen-2-yl)methyl)1,3,2-dioxaborolane (25)

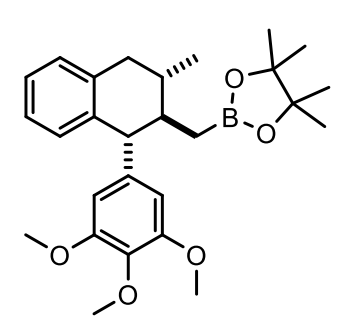

Following the general procedure 2.13, carboxylic acid $\mathbf{2 0}(9.4 \mathrm{mg}$, $50 \mu \mathrm{mol}, 1.0$ equiv.), 1-iodo-3,4,5-trimethoxybenzene (16.2 mg, $55.0 \mu \mathrm{mol}, 1.1$ equiv.), $\mathrm{Cs}_{2} \mathrm{CO}_{3}(19.5 \mathrm{mg}, 60.0 \mu \mathrm{mol}, 1.2$ equiv.), $\operatorname{Pd}(\mathrm{dba})_{2}(5.8 \mathrm{mg}, 20 \mathrm{~mol} \%)$ and toluene $(1.5 \mathrm{~mL})$ were used for the first step. The second step was conducted using triethylsilane $(17.5 \mathrm{mg}$, $0.15 \mathrm{mmol}, 24 \mu \mathrm{L}, 3.0$ equiv.), trichloroborane in $\mathrm{CH}_{2} \mathrm{Cl}_{2}(1 \mathrm{M}, 150 \mu \mathrm{L}, 3.0$ equiv.), toluene (3.0 mL) and 2,2-dimethylpropane-1,3-diol (26 mg, $0.25 \mathrm{mmol}, 5.0$ equiv.). The final transformation was made using THF $(2.5 \mathrm{~mL})$ chloro iodomethane $(88 \mathrm{mg}, 0.50 \mathrm{mmol}$, $36 \mu \mathrm{L}, 10.0$ equiv.), $n$-butyllithium in hexanes ( $1.6 \mathrm{M}, 250 \mu \mathrm{L}, 8.0$ equiv.), aqueous sodium hydroxide $(5.0 \mathrm{~mL})$ and pinacol $(58 \mathrm{mg}, 0.50 \mathrm{mmol}, 10.0$ equiv.). The product was isolated as a colorless oil with a dr. of 4.3:1. (11.3 mg, $25 \mu \mathrm{mol}, 50 \%)$

${ }^{1} \mathbf{H}$ NMR $\left(\mathbf{C D}_{2} \mathbf{C l}_{2}, 500 \mathrm{MHz}\right)$ (major) $\delta 7.09-7.00(\mathrm{~m}, 2 \mathrm{H}), 6.98-6.91(\mathrm{~m}, 1 \mathrm{H}), 6.68(\mathrm{~d}, J$ $=7.8 \mathrm{~Hz}, 1 \mathrm{H}), 6.39$ (s, 2H), $3.86(\mathrm{~d}, J=10.7 \mathrm{~Hz}, 1 \mathrm{H}), 3.77$ (s, 9H), $2.83(\mathrm{dd}, J=16.4,4.4 \mathrm{~Hz}$, $1 \mathrm{H}), 2.66(\mathrm{dd}, J=16.2,11.4 \mathrm{~Hz}, 1 \mathrm{H}), 1.94-1.87(\mathrm{~m}, 1 \mathrm{H}), 1.85-1.78(\mathrm{~m}, 1 \mathrm{H}), 1.21(\mathrm{~s}, 6 \mathrm{H})$, $1.20(\mathrm{~s}, 6 \mathrm{H}), 1.08(\mathrm{~d}, J=6.4 \mathrm{~Hz}, 3 \mathrm{H}), 0.94(\mathrm{dd}, J=16.1,4.3 \mathrm{~Hz}, 1 \mathrm{H}), 0.68(\mathrm{dd}, J=16.0,5.0$ $\mathrm{Hz}, 1 \mathrm{H})$.

${ }^{1} \mathrm{H}$ NMR (CD $\left.\mathbf{C l}_{2}, \mathbf{5 0 0} \mathbf{M H z}\right)$ (minor) $\delta 7.13(\mathrm{~d}, J=7.2 \mathrm{~Hz}, 1 \mathrm{H}), 7.12-7.08(\mathrm{~m}, 1 \mathrm{H}), 7.03$ $7.00(\mathrm{~m}, 1 \mathrm{H}), 6.91(\mathrm{~d}, J=7.6 \mathrm{~Hz}, 1 \mathrm{H}), 6.23(\mathrm{~s}, 2 \mathrm{H}), 4.09$ (d, $J=4.5 \mathrm{~Hz}, 1 \mathrm{H}), 3.72(\mathrm{~s}, 9 \mathrm{H})$, $3.05(\mathrm{dd}, J=17.1,5.1 \mathrm{~Hz}, 1 \mathrm{H}), 2.52(\mathrm{dd}, J=17.1,9.4 \mathrm{~Hz}, 1 \mathrm{H}), 2.03-1.94(\mathrm{~m}, 2 \mathrm{H}), 1.25$ (s, $12 \mathrm{H}), 0.98(\mathrm{~d}, J=6.2 \mathrm{~Hz}, 3 \mathrm{H}), 0.83(\mathrm{dd}, J=15.8,4.9 \mathrm{~Hz}, 1 \mathrm{H}), 0.51(\mathrm{dd}, J=15.6,9.3 \mathrm{~Hz}$, $1 \mathrm{H})$.

${ }^{13} \mathbf{C}\left\{{ }^{1} \mathbf{H}\right\}$ NMR (CD $\mathbf{C D}_{2}$, 125 MHz) (major): $\delta$ 153.7, 142.7, 141.3, 137.7, 136.8, 130.2, 128.6, 126.0, 125.9, 107.4, 83.3, 61.0, 56.5, 54.4, 45.6, 40.0, 35.0, 30.3, 25.3, 25.2, 20.7 ${ }^{13} \mathbf{C}\left\{{ }^{1} \mathbf{H}\right\}$ NMR (CD $\mathbf{C D}_{2}$, 125 MHz) (minor): $\delta$ 152.9, 140.6, 139.9, 137.3, 136.8, 130.4, 129.1, 126.5, 126.0, 108.9, 83.5, 60.9, 56.5, 51.2, 41.8, 38.6, 29.8, 25.6, 25.1, 20.3.

HRMS (ESI): Calculated for $\mathrm{C}_{27} \mathrm{H}_{37} \mathrm{BO}_{5} \mathrm{Na}^{+}: \mathrm{m} / z=475.2626\left([\mathrm{M}+\mathrm{Na}]^{+}\right)$, found: 475.2625 . 


\subsubsection{Rac-2-(((1S,2R,3S)-1-(2,4-dimethoxyphenyl)-3-methyl-}

1,2,3,4-tetrahydronaphthalen-2-yl)methyl)-4,4,5,5-tetramethyl1,3,2-dioxaborolane (26)

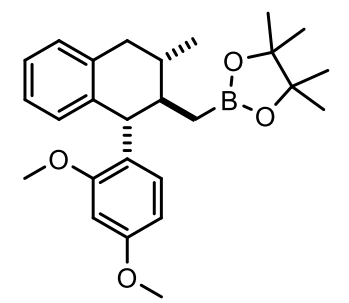

Following the general procedure 2.13 , carboxylic acid $\mathbf{2 0}(9.4 \mathrm{mg}$,

$50 \mu \mathrm{mol}, 1.0$ equiv.), 1-iodo-2,4-dimethox ybenzene (14.5 mg, $55.0 \mu \mathrm{mol}, 1.1$ equiv.), $\mathrm{Cs}_{2} \mathrm{CO}_{3}(19.5 \mathrm{mg}, 60.0 \mu \mathrm{mol}, 1.2$ equiv.), $\operatorname{Pd}(\mathrm{dba})_{2}(5.8 \mathrm{mg}, 20 \mathrm{~mol} \%)$ and toluene $(1.5 \mathrm{~mL})$ were used for the first step. The second step was conducted using triethylsilane $(17.5 \mathrm{mg}$, $0.15 \mathrm{mmol}, 24 \mu \mathrm{L}, 3.0$ equiv.), trichloroborane in $\mathrm{CH}_{2} \mathrm{Cl}_{2}(1 \mathrm{M}, 150 \mu \mathrm{L}, 3.0$ equiv.), toluene (3.0 mL) and 2,2-dimethylpropane-1,3-diol (26 mg, $0.25 \mathrm{mmol}, 5.0$ equiv.). The final transformation was made using THF $(2.5 \mathrm{~mL})$ chloro iodomethane $(88 \mathrm{mg}, 0.50 \mathrm{mmol}$, $36 \mu \mathrm{L}, 10.0$ equiv.), $n$-butyllithium in hexanes ( $1.6 \mathrm{M}, 250 \mu \mathrm{L}, 8.0$ equiv.), aqueous sodium hydroxide $(5.0 \mathrm{~mL})$ and pinacol $(58 \mathrm{mg}, 0.50 \mathrm{mmol}, 10.0$ equiv.). The product was isolated as a colorless oil with a dr. of 7.6:1. (11.6 mg, $27.5 \mu \mathrm{mol}, 55 \%)$

${ }^{1} \mathrm{H}$ NMR ( $\left.\mathrm{CD}_{2} \mathrm{Cl}_{2}, 600 \mathrm{MHz}\right)$ (major isomer) $\delta 7.03(\mathrm{~d}, J=7.5 \mathrm{~Hz}, 1 \mathrm{H}), 7.00-6.97(\mathrm{~m}$, 1H), $6.92-6.88(\mathrm{~m}, 2 \mathrm{H}), 6.58-6.56(\mathrm{~m}, 1 \mathrm{H}), 6.49(\mathrm{~d}, J=2.5 \mathrm{~Hz}, 1 \mathrm{H}), 6.44(\mathrm{dd}, J=8.4,2.5$ Hz, 1H), 4.22 (d, $J=10.1 \mathrm{~Hz}, 1 \mathrm{H}), 3.79$ (s, 3H), 3.72 (s, 3H), 2.77 (dd, $J=16.1,4.1 \mathrm{~Hz}, 1 \mathrm{H})$, $2.64(\mathrm{dd}, J=15.8,11.2 \mathrm{~Hz}, 1 \mathrm{H}), 1.96-1.85(\mathrm{~m}, 2 \mathrm{H}), 1.19(\mathrm{~s}, 6 \mathrm{H}), 1.18(\mathrm{~s}, 6 \mathrm{H}), 1.05(\mathrm{~d}, J=$ $6.3 \mathrm{~Hz}, 3 \mathrm{H}), 0.87$ (dd, $J=16.3,4.0 \mathrm{~Hz}, 1 \mathrm{H}), 0.67$ (dd, $J=16.3,5.4 \mathrm{~Hz}, 1 \mathrm{H})$.

${ }^{1} \mathrm{H}$ NMR (CD $\left.\mathbf{C l}_{2}, 600 \mathrm{MHz}\right)$ (minor isomer) $\delta 7.11-7.09(\mathrm{~m}, 1 \mathrm{H}), 7.07-7.04(\mathrm{~m}, 1 \mathrm{H})$, $6.98-6.96(\mathrm{~m}, 1 \mathrm{H}), 6.82(\mathrm{~d}, J=7.7 \mathrm{~Hz}, 1 \mathrm{H}), 6.56(\mathrm{~d}, J=8.5 \mathrm{~Hz}, 1 \mathrm{H}), 6.47(\mathrm{~d}, J=2.5 \mathrm{~Hz}$, 1H), $6.30(\mathrm{dd}, J=8.5,2.5 \mathrm{~Hz}, 1 \mathrm{H}), 4.69$ (d, $J=4.8 \mathrm{~Hz}, 1 \mathrm{H}), 3.84$ (s, 3H), 3.74 (s, 3H), 3.03 (dd, $J=17.1,5.2 \mathrm{~Hz}, 1 \mathrm{H}), 2.50(\mathrm{dd}, J=17.1,9.0 \mathrm{~Hz}, 1 \mathrm{H}), 2.06-1.96(\mathrm{~m}, 2 \mathrm{H}), 1.20(\mathrm{~s}, 12 \mathrm{H})$, $0.98(\mathrm{~d}, J=6.4 \mathrm{~Hz}, 3 \mathrm{H}), 0.69-0.64(\mathrm{~m}, 1 \mathrm{H}), 0.52(\mathrm{dd}, J=16.3,7.6 \mathrm{~Hz}, 1 \mathrm{H})$.

${ }^{13} \mathbf{C}\left\{{ }^{1} \mathbf{H}\right\}$ NMR ( $\mathbf{C D}_{2} \mathbf{C l}_{2}, \mathbf{1 5 0} \mathbf{M H z}$ (major): 159.7, 159.6, 142.3, 137.9, 131.4, 129.5, 128.4, 128.1, 125.8, 125.4, 105.4, 99.1, 83.2, 56.1, 55.8, 46.8, 44.6, 40.1, 35.2, 25.3, 25.1, 20.8. ${ }^{13} \mathbf{C}\left\{{ }^{1} \mathrm{H}\right\}$ NMR (CD $\left.\mathbf{C l}_{2}, 150 \mathrm{MHz}\right)$ (minor): 158.8, 158.4, 140.9, 136.9, 132.5, 129.7, 128.5, 125.3, 125.2, 124.9, 124.7, 104.8, 103.7, 97.9, 82.6, 55.4, 55.1, 40.7, 40.3, 37.4, 29.6, 29.6, 24.9, 24.4, 19.6.

HRMS (ESI): Calculated for $\mathrm{C}_{26} \mathrm{H}_{35} \mathrm{BO}_{4} \mathrm{Na}^{+}: m / z=445.2525\left([\mathrm{M}+\mathrm{Na}]^{+}\right)$, found: 445.2520 . 
2.13.3. Rac-2-(((1S,2R,3S)-2'-methoxy-3-methyl-1,2,3,4-tetrahydro[1,1'-binaphthalen]-2-yl)methyl)-4,4,5,5-tetramethyl-1,3,2dioxaborolane (27)

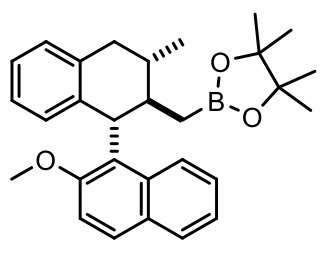

Following the general procedure 2.13, carboxylic acid $\mathbf{2 0}(9.4 \mathrm{mg}$,

$50 \mu \mathrm{mol}, 1.0$ equiv.), 1-iodo-2-methoxynaphthalene (15.6 mg, $55 \mu \mathrm{mol}$, 1.1 equiv.), $\mathrm{Cs}_{2} \mathrm{CO}_{3}$ (19.5 mg, $60.0 \mu \mathrm{mol}, 1.2$ equiv.), $\mathrm{Pd}(\mathrm{dba})_{2}(5.8 \mathrm{mg}$, $20 \mathrm{~mol} \%)$ and toluene $(1.5 \mathrm{~mL})$ were used for the first step. The second step was conducted using triethylsilane (17.5 mg, $0.15 \mathrm{mmol}, 24 \mu \mathrm{L}, 3.0$ equiv.), trichloroborane in $\mathrm{CH}_{2} \mathrm{Cl}_{2}(1 \mathrm{M}, 150 \mu \mathrm{L}, 3.0$ equiv.), toluene $(3.0 \mathrm{~mL})$ and 2,2dimethylpropane-1,3-diol ( $26 \mathrm{mg}, 0.25 \mathrm{mmol}, 5.0$ equiv.). The final transformation was made using THF (2.5 mL) chloroiodomethane ( $88 \mathrm{mg}, 0.50 \mathrm{mmol}, 36 \mu \mathrm{L}, 10.0$ equiv.), $n$ butyllithium in hexanes $(1.6 \mathrm{M}, 250 \mu \mathrm{L}, 8.0$ equiv.), aqueous sodium hydroxide $(5.0 \mathrm{~mL})$ and pinacol (58 mg, $0.50 \mathrm{mmol}, 10.0$ equiv.). The product was isolated as a colorless oil with a dr. of 1:1.3. (6.5 mg, $15 \mu \mathrm{mol}, 30 \%)$

${ }^{1} \mathrm{H}$ NMR $\left(\mathrm{CD}_{2} \mathrm{Cl}_{2}, 600 \mathrm{MHz}\right)$ (major isomer) $\delta 7.79(\mathrm{~d}, J=9.1 \mathrm{~Hz}, 1 \mathrm{H}), 7.75(\mathrm{~d}, J=8.0 \mathrm{~Hz}$, $1 \mathrm{H}), 7.46(\mathrm{~d}, J=8.5 \mathrm{~Hz}, 1 \mathrm{H}), 7.40-7.36(\mathrm{~m}, 1 \mathrm{H}), 7.24(\mathrm{~d}, J=8.9 \mathrm{~Hz}, 1 \mathrm{H}), 7.19$ (t, $J=7.1$ Hz, 1H), $7.15-7.10(\mathrm{~m}, 1 \mathrm{H}), 7.00(\mathrm{t}, J=7.4 \mathrm{~Hz}, 1 \mathrm{H}), 6.80(\mathrm{dt}, J=14.8,7.6 \mathrm{~Hz}, 1 \mathrm{H}), 6.53$ (d, $J=7.8 \mathrm{~Hz}, 1 \mathrm{H}), 5.12(\mathrm{~d}, J=11.0 \mathrm{~Hz}, 1 \mathrm{H}), 3.98(\mathrm{~s}, 3 \mathrm{H}), 2.93(\mathrm{dd}, J=16.4,4.4 \mathrm{~Hz}, 1 \mathrm{H}), 2.87$ $(\mathrm{dd}, J=16.0,11.4 \mathrm{~Hz}, 1 \mathrm{H}), 2.34-2.27(\mathrm{~m}, 1 \mathrm{H}), 2.15-2.07(\mathrm{~m}, 1 \mathrm{H}), 1.14(\mathrm{~s}, 6 \mathrm{H}), 1.11(\mathrm{~d}, J$ $=6.6 \mathrm{~Hz}, 3 \mathrm{H}), 1.09(\mathrm{~s}, 6 \mathrm{H}), 0.81(\mathrm{dd}, J=16.5,4.7 \mathrm{~Hz}, 1 \mathrm{H}), 0.65(\mathrm{dd}, J=16.5,5.5 \mathrm{~Hz}, 1 \mathrm{H})$. ${ }^{1} \mathrm{H}$ NMR (CD $\left.\mathbf{C l}_{2}, 600 \mathrm{MHz}\right)$ (minor isomer) $\delta 8.42(\mathrm{~d}, J=8.7 \mathrm{~Hz}, 1 \mathrm{H}), 7.83(\mathrm{~d}, J=8.5 \mathrm{~Hz}$, 1H), $7.79(\mathrm{~d}, J=9.1 \mathrm{~Hz}, 1 \mathrm{H}), 7.51-7.47(\mathrm{~m}, 1 \mathrm{H}), 7.40-7.36(\mathrm{~m}, 1 \mathrm{H}), 7.15-7.10(\mathrm{~m}, 1 \mathrm{H})$, $7.06(\mathrm{~d}, J=7.6 \mathrm{~Hz}, 1 \mathrm{H}), 6.96(\mathrm{t}, J=7.4 \mathrm{~Hz}, 1 \mathrm{H}), 6.84-6.76(\mathrm{~m}, 1 \mathrm{H}), 6.42(\mathrm{~d}, J=7.8 \mathrm{~Hz}$, $1 \mathrm{H}), 4.96(\mathrm{~d}, J=10.3 \mathrm{~Hz}, 1 \mathrm{H}), 3.39(\mathrm{~s}, 3 \mathrm{H}), 2.82-2.72(\mathrm{~m}, 2 \mathrm{H}), 2.43-2.36(\mathrm{~m}, 1 \mathrm{H}), 2.03-$ $1.95(\mathrm{~m}, 1 \mathrm{H}), 1.22(\mathrm{~s}, 6 \mathrm{H}), 1.18(\mathrm{~s}, 6 \mathrm{H}), 1.09(\mathrm{~d}, J=6.7 \mathrm{~Hz}, 3 \mathrm{H}), 0.88(\mathrm{dd}, J=16.4,5.1 \mathrm{~Hz}$, $1 \mathrm{H}), 0.54(\mathrm{dd}, J=16.4,4.7 \mathrm{~Hz}, 1 \mathrm{H})$.

${ }^{13} \mathbf{C}\left\{{ }^{1} \mathbf{H}\right\}$ NMR $\left(\mathbf{C D}_{2} \mathbf{C l}_{2}, 150 \mathrm{MHz}\right)$ (major and minor) $\delta 157.0,155.8,142.8,142.0,138.3$, 137.5, 135.8, 132.4, 131.1, 130.5, 129.9, 129.2, 129.2, 129.0, 128.8, 128.7, 128.6, 128.1, 128.0, 127.0, 126.9, 126.4, 126.3, 125.7, 125.5, 125.4, 125.0, 124.5, 123.9, 123.3, 116.8, 114.1, 83.3, 83.1, 57.5, 56.9, 44.8, 44.2, 42.9, 42.5, 40.3, 40.2, 35.8, 35.5, 32.5, 30.3, 25.2, 25.1, 25.0, 20.8, 20.6.

HRMS (ESI): Calculated for $\mathrm{C}_{29} \mathrm{H}_{35} \mathrm{BO}_{3} \mathrm{Na}^{+}: m / z=465.2571\left([\mathrm{M}+\mathrm{Na}]^{+}\right)$, found: 465.2572 . 


\subsubsection{Rac-2-(((1R,2R,3S)-1-(3,5-dimethylphenyl)-3-methyl-}

1,2,3,4-tetrahydronaphthalen-2-yl)methyl)-4,4,5,5-tetramethyl1,3,2-dioxaborolane (28)

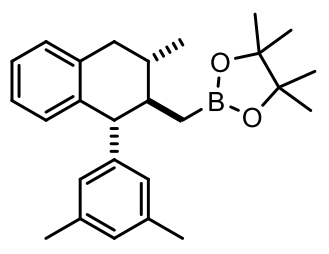

Following the general procedure 2.13, carboxylic acid $\mathbf{2 0}(9.4 \mathrm{mg}$, $50 \mu$ mol, 1.0 equiv.), 1-iodo-3,5-dimethylbenzene (11.6 mg, $55.0 \mu \mathrm{mol}$, 1.1 equiv.), $\mathrm{Cs}_{2} \mathrm{CO}_{3}$ (19.5 mg, $60.0 \mu \mathrm{mol}, 1.2$ equiv.), $\mathrm{Pd}(\mathrm{dba})_{2}(5.8 \mathrm{mg}$, $20 \mathrm{~mol} \%)$ and toluene $(1.5 \mathrm{~mL})$ were used for the first step. The second step was conducted using triethylsilane ( $17.5 \mathrm{mg}, 0.15 \mathrm{mmol}, 24 \mu \mathrm{L}, 3.0$ equiv.), trichloroborane in $\mathrm{CH}_{2} \mathrm{Cl}_{2}(1 \mathrm{M}, 150 \mu \mathrm{L}, 3.0$ equiv. $)$, toluene $(3.0 \mathrm{~mL})$ and 2,2dimethylpropane-1,3-diol ( $26 \mathrm{mg}, 0.25 \mathrm{mmol}, 5.0$ equiv.). The final transformation was made using THF (2.5 mL), chloroiodomethane ( $88 \mathrm{mg}, 0.50 \mathrm{mmol}, 36 \mu \mathrm{L}, 10.0$ equiv.), $n$ butyllithium in hexanes $(1.6 \mathrm{M}, 250 \mu \mathrm{L}, 8.0$ equiv.), aqueous sodium hydroxide $(5.0 \mathrm{~mL})$ and pinacol (58 mg, $0.50 \mathrm{mmol}, 10.0$ equiv.). The product was isolated as a colorless oil with a dr. of 10.5:1. (9.3 mg, $24 \mu \mathrm{mol}, 48 \%)$

${ }^{1} \mathrm{H}$ NMR (CD $\left.\mathrm{Cl}_{2}, 500 \mathrm{MHz}\right)$ (major isomer): $\delta 7.07-7.00(\mathrm{~m}, 2 \mathrm{H}), 6.92(\mathrm{t}, J=7.4 \mathrm{~Hz}$, $1 \mathrm{H}), 6.86(\mathrm{~s}, 1 \mathrm{H}), 6.76(\mathrm{~s}, 2 \mathrm{H}), 6.62-6.60(\mathrm{~m}, 1 \mathrm{H}), 3.83(\mathrm{~d}, J=10.6 \mathrm{~Hz}, 1 \mathrm{H}), 2.81(\mathrm{dd}, J=$ $16.3,4.3 \mathrm{~Hz}, 1 \mathrm{H}), 2.66(\mathrm{dd}, J=16.1,11.6 \mathrm{~Hz}, 1 \mathrm{H}), 2.27$ (s, 6H), $1.94-1.87(\mathrm{~m}, 1 \mathrm{H}), 1.83-$ $1.77(\mathrm{~m}, 1 \mathrm{H}), 1.21(\mathrm{~s}, 6 \mathrm{H}), 1.20(\mathrm{~s}, 6 \mathrm{H}), 1.07$ (d, $J=6.5 \mathrm{~Hz}, 3 \mathrm{H}), 0.92$ (dd, $J=16.2,4.3 \mathrm{~Hz}$, $1 \mathrm{H}), 0.65$ (dd, $J=16.2,5.3 \mathrm{~Hz}, 1 \mathrm{H})$.

${ }^{13} \mathbf{C}\left\{{ }^{1} \mathbf{H}\right\}$ NMR ( $\left.\mathbf{C D}_{2} \mathbf{C l}_{2}, 125 \mathrm{MHz}\right)$ (major isomer): 146.6, 141.2, 137.6, 137.2, 129.8, 128.0, $127.5,125.3,125.2,82.7,53.3,45.1,39.5,34.4,24.6,24.5,21.0,20.1$.

The minor isomer was not fully integrated due to the high diastereomeric ratio.

HRMS (in PhMe, APCI): Calculated for $\mathrm{C}_{26} \mathrm{H}_{35} \mathrm{O}_{2} \mathrm{~B}^{+\bullet}: \mathrm{m} / z=390.2729$ ([M] ${ }^{+*}$ ), found: 390.2721 .

\subsubsection{Rac-2-(((1S,2R,3S)-1-(dibenzo[b,d]thiophen-4-yl)-3-methyl- 1,2,3,4-tetrahydronaphthalen-2-yl)methyl)-4,4,5,5-tetramethyl- 1,3,2-dioxaborolane (29)}

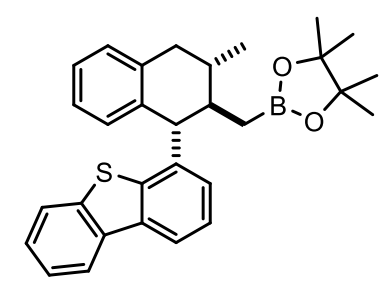

Following the general procedure 2.13, carboxylic acid $\mathbf{2 0}(18.8 \mathrm{mg}$, $100 \mu \mathrm{mol}, 1.0$ equiv.), 1-iodo-3,4,5-trimethoxybenzene (16.2 mg, $110 \mu$ mol, 1.1 equiv.), $\mathrm{Cs}_{2} \mathrm{CO}_{3}(39.0 \mathrm{mg}, 120 \mu \mathrm{mol}, 1.2$ equiv.), $\operatorname{Pd}(\mathrm{dba})_{2}(11.6 \mathrm{mg}, 20 \mathrm{~mol} \%)$ and toluene $(3 \mathrm{~mL})$ were used for the first step. The second step was conducted using triethylsilane $(35.0 \mathrm{mg}, 0.30 \mathrm{mmol}, 48 \mu \mathrm{L}$, 3.0 equiv.), trichloroborane in $\mathrm{CH}_{2} \mathrm{Cl}_{2}(1 \mathrm{M}, 300 \mu \mathrm{L}, 3.0$ equiv. $)$, toluene $(6.0 \mathrm{~mL})$ and 2,2- 
dimethylpropane-1,3-diol (52 mg, $0.50 \mathrm{mmol}, 5.0$ equiv.). The final transformation was made using THF (5.0 mL) chloro iodomethane ( $0.18 \mathrm{~g}, 1.0 \mathrm{mmol}, 72 \mu \mathrm{L}, 10$ equiv. $), n$-butyllithium in hexanes ( $1.6 \mathrm{M}, 500 \mu \mathrm{L}, 8.0$ equiv.), aqueous sodium hydroxide $(10.0 \mathrm{~mL})$ and pinacol (116 mg, $1.00 \mathrm{mmol}, 10.0$ equiv.). The product was isolated as a colorless oil with a dr. of 7.4:1 (22.4 mg, $48.0 \mu \mathrm{mol}, 48 \%)$

${ }^{1} \mathrm{H}$ NMR (CD $\mathbf{C l}_{2}, 600 \mathrm{MHz}$ ) (major isomer): $\delta 8.19-8.14(\mathrm{~m}, 1 \mathrm{H}), 8.09$ (dd, $J=7.9,1.1$ $\mathrm{Hz}, 1 \mathrm{H}), 7.79-7.74(\mathrm{~m}, 1 \mathrm{H}), 7.49-7.39$ (m, 3H), $7.37-7.32(\mathrm{~m}, 1 \mathrm{H}), 7.15-7.13(\mathrm{~m}, 1 \mathrm{H})$, $7.08-7.04(\mathrm{~m}, 1 \mathrm{H}), 6.86$ (t, $J=7.5 \mathrm{~Hz}, 1 \mathrm{H}), 6.63(\mathrm{~d}, J=7.8 \mathrm{~Hz}, 1 \mathrm{H}), 4.38(\mathrm{~d}, J=10.9 \mathrm{~Hz}$, $1 \mathrm{H}), 2.89(\mathrm{dd}, J=16.5,4.4 \mathrm{~Hz}, 1 \mathrm{H}), 2.80(\mathrm{dd}, J=16.6,11.7 \mathrm{~Hz}, 1 \mathrm{H}), 2.27-2.20(\mathrm{~m}, 1 \mathrm{H})$, $2.08-1.99(\mathrm{~m}, 1 \mathrm{H}), 1.21(\mathrm{~s}, 6 \mathrm{H}), 1.18(\mathrm{~s}, 6 \mathrm{H}), 1.11(\mathrm{~d}, J=6.6 \mathrm{~Hz}, 3 \mathrm{H}), 0.95(\mathrm{dd}, J=16.4$, $4.5 \mathrm{~Hz}, 1 \mathrm{H}), 0.67(\mathrm{dd}, J=16.4,5.1 \mathrm{~Hz}, 1 \mathrm{H})$.

${ }^{1} \mathbf{H}$ NMR ( $\left.\mathbf{C D}_{2} \mathbf{C l}_{2}, 600 \mathrm{MHz}\right)$ (minor isomer): $\delta 8.19-8.14(\mathrm{~m}, 1 \mathrm{H}), 8.05-8.03(\mathrm{~m}, 1 \mathrm{H})$, $7.87(\mathrm{dd}, J=5.8,3.2 \mathrm{~Hz}, 1 \mathrm{H}), 7.48-7.40(\mathrm{~m}, 2 \mathrm{H}), 7.36-7.32(\mathrm{~m}, 1 \mathrm{H}), 7.18(\mathrm{~d}, J=8.1 \mathrm{~Hz}$, 1H), $7.15-7.12(\mathrm{~m}, 1 \mathrm{H}), 7.08-7.04(\mathrm{~m}, 1 \mathrm{H}), 7.01-6.98(\mathrm{~m}, 1 \mathrm{H}), 6.93(\mathrm{~d}, J=7.7 \mathrm{~Hz}, 1 \mathrm{H})$, $4.65(\mathrm{~d}, J=5.6 \mathrm{~Hz}, 1 \mathrm{H}), 3.16(\mathrm{dd}, J=16.9,5.5 \mathrm{~Hz}, 1 \mathrm{H}), 2.58(\mathrm{dd}, J=17.2,7.4 \mathrm{~Hz}, 1 \mathrm{H}), 2.37$ $-2.31(\mathrm{~m}, 1 \mathrm{H}), 2.28-2.22(\mathrm{~m}, 1 \mathrm{H}), 1.14(\mathrm{~s}, 6 \mathrm{H}), 1.12(\mathrm{~s}, 6 \mathrm{H}), 1.11(\mathrm{~d}, J=6.7 \mathrm{~Hz}, 3 \mathrm{H}), 0.75$ $-0.70(\mathrm{~m}, 1 \mathrm{H}), 0.63-0.58(\mathrm{~m}, 1 \mathrm{H})$.

${ }^{13} \mathbf{C}\left\{{ }^{1} \mathrm{H}\right\}$ NMR (CD $\left.\mathbf{C D}_{2}, 150 \mathrm{MHz}\right)$ (major and minor): $\delta 141.7,140.2,139.8,139.3,138.5$, 137.7, 137.1, 136.7, 136.4, 130.5, 129.8, 129.7, 129.5, 129.2, 128.8, 127.2, 127.2, 126.7, 126.3, 126.2, 126.1, 125.3, 125.0, 124.9, 124.8, 124.7, 123.1, 123.0, 122.1, 120.2, 120.0, 83.4, 53.9, 49.5, 43.2, 40.6, 40.1, 36.5, 36.1, 34.9, 31.3, 30.3, 25.3, 25.2, 25.2, 25.1, 23.3, 20.6, 20.2 .

HRMS (ESI): Calculated for $\mathrm{C}_{30} \mathrm{H}_{33} \mathrm{BO}_{2} \mathrm{SNa}^{+}: m / z=491.2187\left([\mathrm{M}+\mathrm{Na}]^{+}\right)$, found: 491.2189 .

\subsection{Protodeboronation: General procedure for the synthesis of compounds 30 to 34}

The boronic ester (1.0 equiv.) was dissolved in THF and cooled to $-78{ }^{\circ} \mathrm{C}$ before phenyllithium in di- $n$-butylether (1.9M, 1.1 equiv.) was added at once. After stirring for 10 min. the reaction was taken out of the cooling bath and allowed to warm up to rt. The solvent was removed in vacuo and to the residue was added thiophenol (1.1 equiv.) followed by the addition of methanol and acetone in that order. $\operatorname{Ir}\left(\mathrm{dFFppy}_{2}(\mathrm{dtb}-\mathrm{bpy}) \mathrm{PF}_{6}(2 \mathrm{~mol} \%)\right.$ was added in one potion and the mixture degassed via three freeze-pump-thaw degassing cycles and irradiated with a LED $(30 \mathrm{~W}, 460 \mathrm{~nm})$ for $18 \mathrm{~h}$ at rt. After separation via preparative TLC the products were isolated as colorless oils. 


\subsubsection{Rac-(1R,2R,3S)-2,3-dimethyl-1-(3,4,5-trimethoxyphenyl)- 1,2,3,4-tetrahydronaphthalene (30)}

(c)

Following the general procedure 2.14 , boronic ester $\mathbf{2 5}(10.0 \mathrm{mg}, 22 \mu \mathrm{mol}$, 1.0 equiv., $\mathrm{dr}=4.3: 1), \operatorname{THF}(0.5 \mathrm{~mL})$, phenyllithium $(14 \mu \mathrm{L}, 1.1$ equiv. $)$, thiophenol (2.7 mg, $2.6 \mu \mathrm{L}, 1.1$ equiv.), methanol $(0.5 \mathrm{~mL})$, acetone $(0.5 \mathrm{~mL})$ and $\operatorname{Ir}(\mathrm{dFFppy})_{2}(\mathrm{dtb}-\mathrm{bpy}) \mathrm{PF}_{6}(0.54 \mathrm{mg}, 2 \mathrm{~mol} \%)$ were used. After separation by preparative TLC ( $n$-hexane/ethylacetate, 40:1), the product was isolated as a colorless with a dr. of 3.6:1. (6.1 mg, $19 \mu \mathrm{mol}, 85 \%)$.

${ }^{1} \mathrm{H}$ NMR ( $\left.\mathrm{CDCl}_{3}, 600 \mathrm{MHz}\right)$ (major isomer): $\delta 7.08-7.07(\mathrm{~m}, 2 \mathrm{H}), 7.01-6.97(\mathrm{~m}, 1 \mathrm{H})$, $6.72(\mathrm{~d}, J=8.0 \mathrm{~Hz}, 1 \mathrm{H}), 6.33(\mathrm{~s}, 2 \mathrm{H}), 3.86(\mathrm{~s}, 3 \mathrm{H}), 3.80(\mathrm{~s}, 6 \mathrm{H}), 3.48(\mathrm{~d}, J=10.6 \mathrm{~Hz}, 1 \mathrm{H})$, $2.85(\mathrm{dd}, J=16.4,4.5 \mathrm{~Hz}, 1 \mathrm{H}), 2.68(\mathrm{dd}, J=16.5,11.4 \mathrm{~Hz}, 1 \mathrm{H}), 1.72-1.65(\mathrm{~m}, 1 \mathrm{H}), 1.63-$ $1.57(\mathrm{~m}, 1 \mathrm{H}), 1.10(\mathrm{~d}, J=6.4 \mathrm{~Hz}, 3 \mathrm{H}), 0.90(\mathrm{~d}, J=6.4 \mathrm{~Hz}, 3 \mathrm{H})$.

${ }^{1} \mathrm{H}_{\mathrm{NMR}}\left(\mathrm{CDCl}_{\mathbf{3}}, \mathbf{6 0 0} \mathrm{MHz}\right)$ (minor isomer): $\delta 7.17-7.10(\mathrm{~m}, 2 \mathrm{H}), 7.05-7.03(\mathrm{~m}, 1 \mathrm{H})$, $6.95(\mathrm{~d}, J=7.7 \mathrm{~Hz}, 1 \mathrm{H}), 6.19(\mathrm{~s}, 2 \mathrm{H}), 4.03-4.02(\mathrm{~m}, 1 \mathrm{H}), 3.82(\mathrm{~s}, 3 \mathrm{H}), 3.75(\mathrm{~s}, 6 \mathrm{H}), 3.02$ $(\mathrm{dd}, J=16.8,5.3 \mathrm{~Hz}, 1 \mathrm{H}), 2.52(\mathrm{dd}, J=16.9,10.0 \mathrm{~Hz}, 1 \mathrm{H}), 1.94-1.83(\mathrm{~m}, 2 \mathrm{H}), 0.99$ (d, $J=$ $6.3 \mathrm{~Hz}, 3 \mathrm{H}), 0.85(\mathrm{~d}, J=6.7 \mathrm{~Hz}, 3 \mathrm{H})$.

${ }^{13} \mathbf{C}\left\{{ }^{1} \mathrm{H}\right\}$ NMR (CDCl $\mathbf{3}, 150 \mathrm{MHz}$ ) (major isomer): $\delta$ 153.2, 142.2, 140.4, 137.0, 136.5, $129.9,128.3,125.8,125.8,106.7,61.0,56.3,55.6,43.7,39.5,35.6,20.2,17.5$.

${ }^{13} \mathbf{C}\left\{{ }^{1} \mathbf{H}\right\}$ NMR (CDCl $\mathbf{3}, 150 \mathrm{MHz}$ ) (minor isomer): $\delta 152.4,139.7,139.6,136.8,136.4$, $130.4,128.7,126.1,125.8,108.1,61.0,56.2,51.2,39.4,38.2,29.4,19.8,17.4$.

HRMS (ESI): Calculated for $\mathrm{C}_{21} \mathrm{H}_{26} \mathrm{O}_{3} \mathrm{Na}^{+}: m / z=349.1774\left([\mathrm{M}+\mathrm{Na}]^{+}\right)$, found: 349.1774 .

\subsubsection{Rac-(1S,2R,3S)-1-(2,4-dimethoxyphenyl)-2,3-dimethyl- 1,2,3,4-tetrahydronaphthalene (31)}

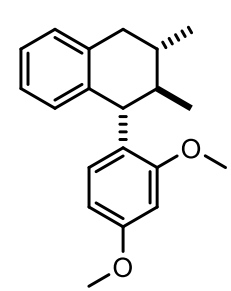

Following the general procedure 2.14, boronic ester $\mathbf{2 6}(11.6 \mathrm{mg}, 27 \mu \mathrm{mol}$, 1.0 equiv.), THF ( $0.5 \mathrm{~mL})$, phenyllithium ( $16 \mu \mathrm{L}, 1.1$ equiv.), thiophenol (3.3 mg, $3.0 \mu \mathrm{L}, 1.1$ equiv.), methanol $(0.5 \mathrm{~mL})$, acetone $(0.5 \mathrm{~mL})$ and $\operatorname{Ir}(\mathrm{dFFppy})_{2}(\mathrm{dtb}-\mathrm{bpy}) \mathrm{PF}_{6}(0.60 \mathrm{mg}, 2 \mathrm{~mol} \%)$ were used. After separation by preparative TLC ( $n$-hexane/ethylacetate, 40:1), the product was isolated as a colorless oil with a dr. of 5.4:1. (4.2 mg, $14 \mu \mathrm{mol}, 52 \%)$.

${ }^{1} \mathrm{H}$ NMR (CDCl $\left.3,600 \mathrm{MHz}\right)$ (major isomer): $\delta 7.05-7.00(\mathrm{~m}, 2 \mathrm{H}), 6.95-6.92(\mathrm{~m}, 1 \mathrm{H})$, $6.89(\mathrm{~d}, J=8.4 \mathrm{~Hz}, 1 \mathrm{H}), 6.64(\mathrm{~d}, J=7.8 \mathrm{~Hz}, 1 \mathrm{H}), 6.49$ (d, $J=2.5 \mathrm{~Hz}, 1 \mathrm{H}), 6.44$ (dd, $J=8.4$, $2.5 \mathrm{~Hz}, 1 \mathrm{H}), 4.05-4.00(\mathrm{~m}, 1 \mathrm{H}), 3.80(\mathrm{~s}, 3 \mathrm{H}), 3.73(\mathrm{~s}, 3 \mathrm{H}), 2.81(\mathrm{dd}, J=16.1,4.0 \mathrm{~Hz}, 1 \mathrm{H})$, 
$2.66(\mathrm{dd}, J=16.4,11.5 \mathrm{~Hz}, 1 \mathrm{H}), 1.72-1.64(\mathrm{~m}, 2 \mathrm{H}), 1.08(\mathrm{~d}, J=6.1 \mathrm{~Hz}, 3 \mathrm{H}), 0.86(\mathrm{~d}, J=$ $6.1 \mathrm{~Hz}, 3 \mathrm{H})$.

${ }^{1} \mathrm{H}$ NMR (CDCl, $\left.600 \mathrm{MHz}\right)$ (minor isomer): $\delta 7.12-7.06(\mathrm{~m}, 2 \mathrm{H}), 7.00-6.98(\mathrm{~m}, 1 \mathrm{H})$, $6.88-6.86(\mathrm{~m}, 1 \mathrm{H}), 6.53(\mathrm{~d}, J=8.5 \mathrm{~Hz}, 1 \mathrm{H}), 6.46(\mathrm{~d}, J=2.5 \mathrm{~Hz}, 1 \mathrm{H}), 6.30(\mathrm{dd}, J=8.5,2.5$ $\mathrm{Hz}, 1 \mathrm{H}), 4.71(\mathrm{~d}, J=5.1 \mathrm{~Hz}, 1 \mathrm{H}), 3.85(\mathrm{~s}, 3 \mathrm{H}), 3.76$ (s, 3H), 2.99 (dd, $J=17.1,5.1 \mathrm{~Hz}, 1 \mathrm{H})$, $2.51(\mathrm{dd}, J=17.1,9.7 \mathrm{~Hz}, 1 \mathrm{H}), 1.92-1.82(\mathrm{~m}, 2 \mathrm{H}), 0.97(\mathrm{~d}, J=6.2 \mathrm{~Hz}, 3 \mathrm{H}), 0.79$ (d, $J=6.6$ $\mathrm{Hz}, 3 \mathrm{H})$.

${ }^{13} \mathbf{C}\left\{{ }^{1} \mathbf{H}\right\}$ NMR (CDCl $3,150 \mathrm{MHz}$ ) (major and minor isomer): $\delta$ 159.1, 159.1, 141.4, 141.1, 137.3, 137.2, 132.6, 130.8, 130.5, 129.3, 128.5, 128.0, 127.9, 125.7, 125.6, 125.6, 125.1, 124.6, 104.9, 103.9, 98.8, 98.0, 55.8, 55.5, 55.4, 55.3, 46.7, 43.1, 40.8, 39.6, 39.4, 38.5, 35.9, 29.1, 20.2, 19.6, 17.3, 15.5.

HRMS (in PhMe, APCI): Calculated for $\mathrm{C}_{20} \mathrm{H}_{24} \mathrm{O}_{2}{ }^{+\bullet}: m / z=296.1771\left([\mathrm{M}]^{+\bullet}\right.$ ), found: 296.1766.

\subsubsection{Rac-(1S,2R,3S)-2'-methoxy-2,3-dimethyl-1,2,3,4- tetrahydro-1,1'-binaphthalene (32)}

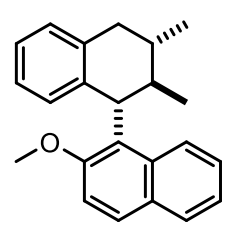

Following the general procedure 2.14, boronic ester 27 (6.4 mg, $14 \mu \mathrm{mol}$, 1.0 equiv., $\mathrm{dr}=1.3: 1)$, THF $(0.4 \mathrm{~mL})$, phenyllithium $(8.4 \mu \mathrm{L}, 1.1$ equiv. $)$, thiophenol (1.7 mg, $1.6 \mu \mathrm{L}, 1.1$ equiv.), methanol $(0.4 \mathrm{~mL})$, acetone $(0.4 \mathrm{~mL})$ and $\operatorname{Ir}(\mathrm{dFFppy})_{2}$ (dtb-bpy) $\mathrm{PF}_{6}(0.31 \mathrm{mg}, 2 \mathrm{~mol} \%)$ was used. After separation by preparative TLC (cyclohexane/ethylacetate, 100:1) the product was isolated as a colorless oil with a dr. of 2.0:1. (3.8 mg, $12 \mu \mathrm{mol}, 85 \%)$.

${ }^{1} \mathrm{H}$ NMR (CDCl $\left.\mathbf{3}, 600 \mathrm{MHz}\right)$ (major isomer): $\delta 7.77(\mathrm{~d}, J=9.0 \mathrm{~Hz}, 1 \mathrm{H}), 7.74(\mathrm{~d}, J=7.6 \mathrm{~Hz}$, 1H), $7.44-7.42(\mathrm{~m}, 1 \mathrm{H}), 7.36(\mathrm{~d}, J=9.0 \mathrm{~Hz}, 1 \mathrm{H}), 7.21-7.18(\mathrm{~m}, 1 \mathrm{H}), 7.16-7.10(\mathrm{~m}, 2 \mathrm{H})$, $7.04-7.01(\mathrm{~m}, 1 \mathrm{H}), 6.86-6.84(\mathrm{~m}, 1 \mathrm{H}), 6.61(\mathrm{~d}, J=7.8 \mathrm{~Hz}, 1 \mathrm{H}), 4.98(\mathrm{~d}, J=11.0 \mathrm{~Hz}, 1 \mathrm{H})$, 3.98 (s, 3H), 2.97 (dd, $J=16.5,4.4 \mathrm{~Hz}, 1 \mathrm{H}), 2.88$ (dd, $J=16.6,11.8 \mathrm{~Hz}, 1 \mathrm{H}), 2.15-2.09$ (m, $1 \mathrm{H}), 1.88-1.81(\mathrm{~m}, 1 \mathrm{H}), 1.12(\mathrm{~d}, J=6.5 \mathrm{~Hz}, 3 \mathrm{H}), 0.80(\mathrm{~d}, J=6.5 \mathrm{~Hz}, 3 \mathrm{H})$.

${ }^{1} \mathrm{H}$ NMR (CDCl, $600 \mathrm{MHz}$ ) (minor isomer): $\delta 8.24(\mathrm{~d}, J=8.6 \mathrm{~Hz}, 1 \mathrm{H}), 7.84(\mathrm{~d}, J=8.4 \mathrm{~Hz}$, $1 \mathrm{H}), 7.78(\mathrm{~d}, J=8.9 \mathrm{~Hz}, 1 \mathrm{H}), 7.54-7.51(\mathrm{~m}, 1 \mathrm{H}), 7.41-7.38(\mathrm{~m}, 1 \mathrm{H}), 7.23-7.20(\mathrm{~m}, 1 \mathrm{H})$, $7.09-7.07(\mathrm{~m}, 1 \mathrm{H}), 7.00-6.98(\mathrm{~m}, 1 \mathrm{H}), 6.83-6.81(\mathrm{~m}, 1 \mathrm{H}), 6.51(\mathrm{~d}, J=7.6 \mathrm{~Hz}, 1 \mathrm{H}), 4.59$ $(\mathrm{d}, J=10.0 \mathrm{~Hz}, 1 \mathrm{H}), 3.35$ (s, 3H), $2.84-2.75(\mathrm{~m}, 2 \mathrm{H}), 2.23-2.17(\mathrm{~m}, 1 \mathrm{H}), 1.77-1.72(\mathrm{~m}$, $1 \mathrm{H}), 1.13-1.11(\mathrm{~m}, 3 \mathrm{H}), 0.85(\mathrm{~d}, J=6.6 \mathrm{~Hz}, 3 \mathrm{H})$.

${ }^{13} \mathbf{C}\left\{{ }^{1} \mathrm{H}\right\}$ NMR (CDCl $\left.3,150 \mathrm{MHz}\right)$ (major and minor isomer): $\delta 156.3,154.9,141.7,140.9$, $137.4,136.3$, 134.8, 131.7, 130.4, 129.9, 129.2, 128.6, 128.5, 128.2, 128.1, 127.5, 127.3, 
$126.6,126.3,126.0,125.9,125.3,125.2,125.0,124.6,123.4,123.3,122.9,116.2,113.6,57.1$, $56.4,45.4,43.9,40.9,40.8,39.6,39.5,36.3,36.2,20.0,19.9,18.2,17.4$.

HRMS (in PhMe, APCI): Calculated for $\mathrm{C}_{23} \mathrm{H}_{24} \mathrm{O}^{+\bullet}: \mathrm{m} / z=316.1821\left([\mathrm{M}]^{+*}\right)$, found: 316.1817.

\subsubsection{Rac-(1R,2R,3S)-1-(3,5-dimethylphenyl)-2,3-dimethyl- 1,2,3,4-tetrahydronaphthalene (33)}<smiles>Cc1cc(C)cc([C@H]2c3ccccc3C[C@H](C)C2C)c1</smiles>

Following the general procedure 2.14, boronic ester $\mathbf{2 8}(9.0 \mathrm{mg}, 23 \mu \mathrm{mol}$, 1.0 equiv., $\mathrm{dr}=10.5: 1)$, THF $(0.5 \mathrm{~mL})$, phenyllithium $(14 \mu \mathrm{L}, 1.1$ equiv. $)$, thiophenol (2.9 mg, $2.7 \mu \mathrm{L}, 1.1$ equiv.), methanol $(0.5 \mathrm{~mL})$, acetone $(0.5 \mathrm{~mL})$ and $\operatorname{Ir}(\mathrm{dFFppy})_{2}(\mathrm{dtb}-\mathrm{bpy}) \mathrm{PF}_{6}(0.54 \mathrm{mg}, 2 \mathrm{~mol} \%)$ was used. After separation by preparative TLC (cyclohexane/ethylacetate, 100:1) the product was isolated as a colorless oil as single diasteromer. (3.0 mg, $11 \mu \mathrm{mol}, 50 \%)$.

${ }^{1} \mathrm{H}$ NMR (CDCl, $\left.600 \mathrm{MHz}\right): \delta 7.09$ - $7.04(\mathrm{~m}, 2 \mathrm{H}), 6.98$ - $6.94(\mathrm{~m}, 1 \mathrm{H}), 6.86-6.85$ (m, $1 \mathrm{H}), 6.74-6.72(\mathrm{~m}, 2 \mathrm{H}), 6.68(\mathrm{~d}, J=8.0 \mathrm{~Hz}, 1 \mathrm{H}), 3.48(\mathrm{~d}, J=10.4 \mathrm{~Hz}, 1 \mathrm{H}), 2.83(\mathrm{dd}, J=$ 16.4, 4.3 Hz, 1H), 2.69 (dd, $J=16.8,12.2 \mathrm{~Hz}, 1 \mathrm{H}), 2.28$ (d, $J=0.6 \mathrm{~Hz}, 6 \mathrm{H}), 1.70-1.58(\mathrm{~m}$, 2H), $1.09(\mathrm{~d}, J=6.3 \mathrm{~Hz}, 3 \mathrm{H}), 0.88(\mathrm{~d}, J=6.2 \mathrm{~Hz}, 3 \mathrm{H})$.

${ }^{13} \mathbf{C}\left\{{ }^{1} \mathbf{H}\right\}$ NMR (CDCl 3 , 150 MHz): $\delta 146.6,140.9,137.7,137.2,130.2,128.2,127.9,127.6$, $125.8,125.5,54.9,43.9,39.6,35.6,21.5,20.2,17.5$.

HRMS (in PhMe, APCI): Calculated for $\mathrm{C}_{20} \mathrm{H}_{24^{+}}: \mathrm{m} / \mathrm{z}=264.1872$ ([M] ${ }^{+\bullet}$ ), found: 264.1867.

\subsubsection{Rac-4-((1S,2R,3S)-2,3-dimethyl-1,2,3,4- tetrahydronaphthalen-1-yl)dibenzo[b,d]thiophene (34)}

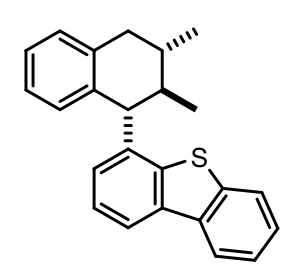

Following the general procedure 2.14 , boronic ester $\mathbf{2 9}(17.5 \mathrm{mg}, 37 \mu \mathrm{mol}$, 1.0 equiv., $\mathrm{dr}=7.4: 1)$, THF $(1 \mathrm{~mL})$, phenyllithium $(21 \mu \mathrm{L}, 1.1$ equiv. $)$, thiophenol (4.5 mg, $4.2 \mu \mathrm{L}, 1.1$ equiv.), methanol (1.0 mL), acetone $(1.0 \mathrm{~mL})$ and $\operatorname{Ir}(\mathrm{dFFppy})_{2}(\mathrm{dtb}-\mathrm{bpy}) \mathrm{PF}_{6}(0.82 \mathrm{mg}, 2 \mathrm{~mol} \%)$ was used. After separation by preparative TLC ( $n$-hexane/ethylacetate, 40:1) the product was isolated as a colorless oil with a dr. of 2.0:1. (5.2 mg, $15 \mu \mathrm{mol}, 41 \%)$.

${ }^{1} \mathrm{H}$ NMR (CDCl, $600 \mathrm{MHz}$ ) (major isomer): $\delta 8.16-8.14(\mathrm{~m}, 1 \mathrm{H}), 8.07$ (dd, $J=7.8,1.1$ $\mathrm{Hz}, 1 \mathrm{H}), 7.78-7.75(\mathrm{~m}, 1 \mathrm{H}), 7.46-7.40(\mathrm{~m}, 3 \mathrm{H}), 7.28(\mathrm{dd}, J=7.4,1.1 \mathrm{~Hz}, 1 \mathrm{H}), 7.16-7.13$ (m, 1H), $7.12-7.07(\mathrm{~m}, 1 \mathrm{H}), 6.91(\mathrm{t}, J=7.6 \mathrm{~Hz}, 1 \mathrm{H}), 6.69(\mathrm{~d}, J=7.9 \mathrm{~Hz}, 1 \mathrm{H}), 4.02(\mathrm{~d}, J=$ $10.7 \mathrm{~Hz}, 1 \mathrm{H}), 2.92$ (dd, $J=16.4,4.6 \mathrm{~Hz}, 1 \mathrm{H}), 2.84$ (dd, $J=16.8,11.8 \mathrm{~Hz}, 1 \mathrm{H}), 2.06-1.98$ $(\mathrm{m}, 1 \mathrm{H}), 1.84-1.75(\mathrm{~m}, 1 \mathrm{H}), 1.14(\mathrm{~d}, J=6.5 \mathrm{~Hz}, 3 \mathrm{H}), 0.92(\mathrm{~d}, J=6.4 \mathrm{~Hz}, 3 \mathrm{H})$. 
${ }^{1} \mathrm{H}$ NMR (CDCl, $600 \mathrm{MHz}$ ) (minor isomer): $\delta 8.16-8.15(\mathrm{~m}, 1 \mathrm{H}), 8.01-7.98(\mathrm{~m}, 1 \mathrm{H})$, $7.89-7.86(\mathrm{~m}, 1 \mathrm{H}), 7.51-7.29(\mathrm{~m}, 3 \mathrm{H}), 7.24-7.21(\mathrm{~m}, 1 \mathrm{H}), 7.18(\mathrm{~d}, J=7.6 \mathrm{~Hz}, 1 \mathrm{H}), 7.00$ $(\mathrm{t}, J=7.8 \mathrm{~Hz}, 1 \mathrm{H}), 6.99-6.91(\mathrm{~m}, 2 \mathrm{H}), 4.59(\mathrm{~d}, J=5.1 \mathrm{~Hz}, 1 \mathrm{H}), 3.12(\mathrm{dd}, J=17.1,4.8 \mathrm{~Hz}$, 1H), $2.60(\mathrm{dd}, J=16.9,8.8 \mathrm{~Hz}, 1 \mathrm{H}), 2.19-2.11(\mathrm{~m}, 2 \mathrm{H}), 1.07(\mathrm{~d}, J=6.1 \mathrm{~Hz}, 3 \mathrm{H}), 0.87$ (d, $J$ $=6.5 \mathrm{~Hz}, 3 \mathrm{H})$.

${ }^{13} \mathbf{C}\left\{{ }^{1} \mathbf{H}\right\}$ NMR (CDCl $\left.3,150 \mathrm{MHz}\right)$ (major and minor isomer): $\delta 141.1,139.7,139.5,139.1$, 139.0, 138.5, 137.7, 136.8, 136.3, 136.1, 135.6, 130.4, 129.6, 128.9, 128.9, 128.4, 128.2, $126.8,126.7,126.3,126.1,126.1,126.0,124.9,124.6,124.5,124.4,122.8,122.8,121.8$, 121.7, 119.8, 119.5, 54.8, 49.5, 41.8, 39.5, 39.5, 37.6, 35.7, 30.0, 20.0, 19.8, 17.7, 16.7 .

HRMS (in PhMe, APCI): Calculated for $\mathrm{C}_{24} \mathrm{H}_{22} \mathrm{~S}^{+\bullet}: \mathrm{m} / z=342.1436\left([\mathrm{M}]^{+\bullet}\right.$ ), found: 342.1430 . 


\section{HPLC data and UV spectrum}

\section{1. (S)-2-(6,7-Dimethoxynaphthaline-2-yl)-4-isopropyl-4,5- dihydrooxazole (6)}

\section{Chromatograms}

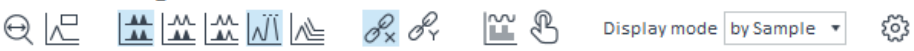
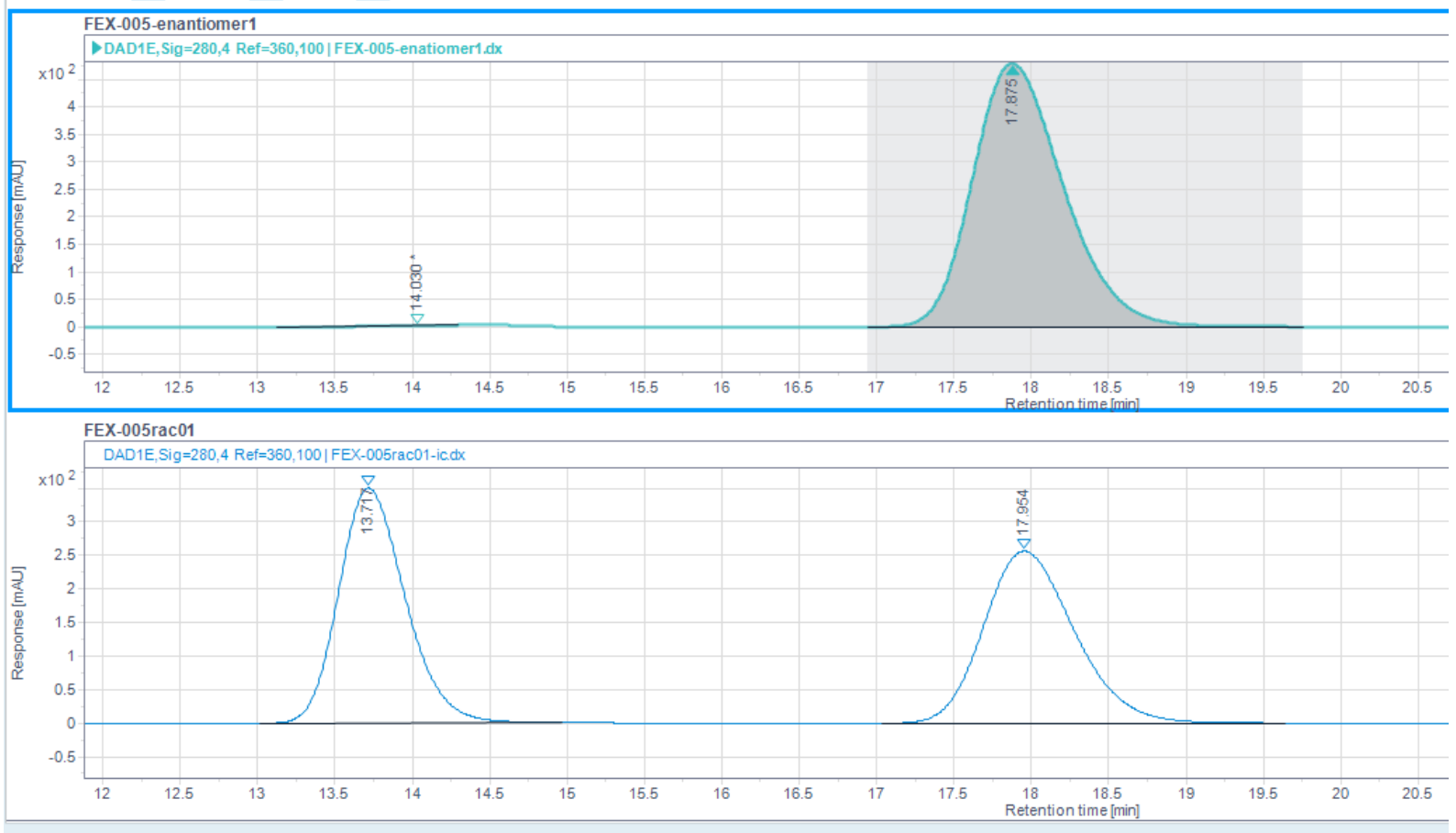

Injection Results

Peaks Summary

\begin{tabular}{|c|c|c|c|c|c|}
\hline \multirow[t]{3}{*}{ एా $\#$} & - Signal description & RT (min) & Area & Area\% & Height \\
\hline & 1 DAD1E,Sig $=280,4$ Ref $=360,100$ & 14.030 & 62.477 & 0.32 & 1.759 \\
\hline & 2 DAD1E,Sig $=280,4$ Ref $=360,100$ & 17.875 & 19585.918 & 99.68 & 478.869 \\
\hline
\end{tabular}

Figure 1: Racemate and enantioenriched sample of 6. 


\section{2. (S)-2-((1S,2S)-1-(Dimethyl(phenyl)silyl)-6,7-dimethoxy-2-methyl-}

1,2-dihydronaphthalene-2-yl)-4-isopropyl-4,5-dihydrooxazole (9)

\section{Chromatograms}

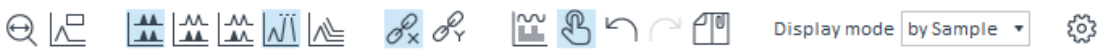
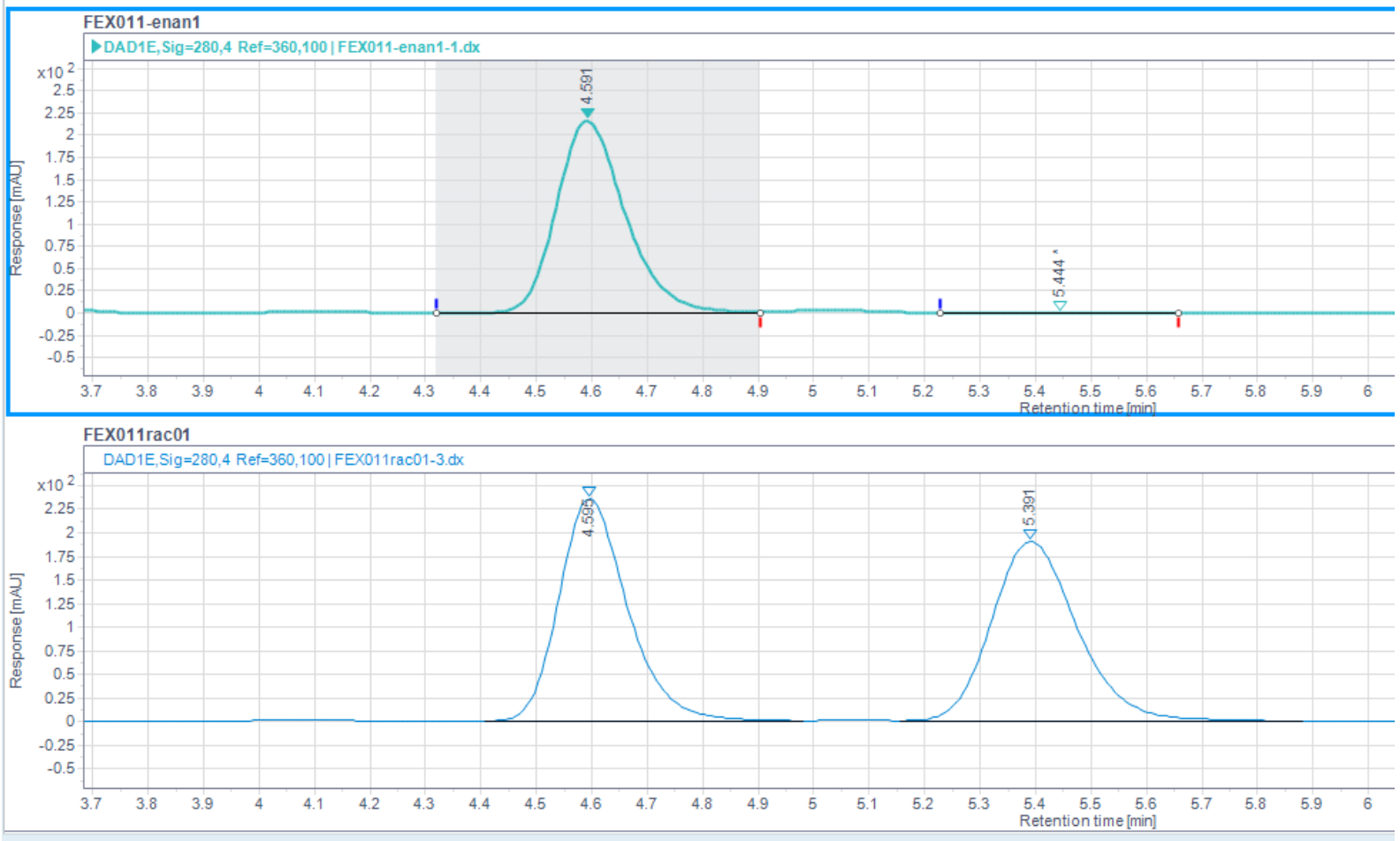

Injection Results

Peaks summary

एా \# - Signal description

1 DAD1E,Sig $=280,4$ Ref $=360,100$

\begin{tabular}{|c|c|c|c|}
\hline RT (min) & Area & Area\% & Height \\
\hline 4.591 & 1868.417 & 99.77 & 216.395 \\
\hline 5.444 & 4.364 & 0.23 & 0.354 \\
\hline
\end{tabular}

Figure 2: Racemate and enantioenriched sample of 9 


\section{3. (S)-Methyl 6,7-Dimethoxy-2-methyl-1,2-dihydronaphthalene-2- carboxylate (5-OMe)}

\section{Chromatograms}

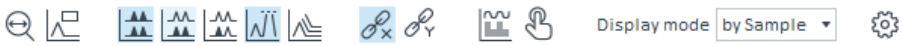
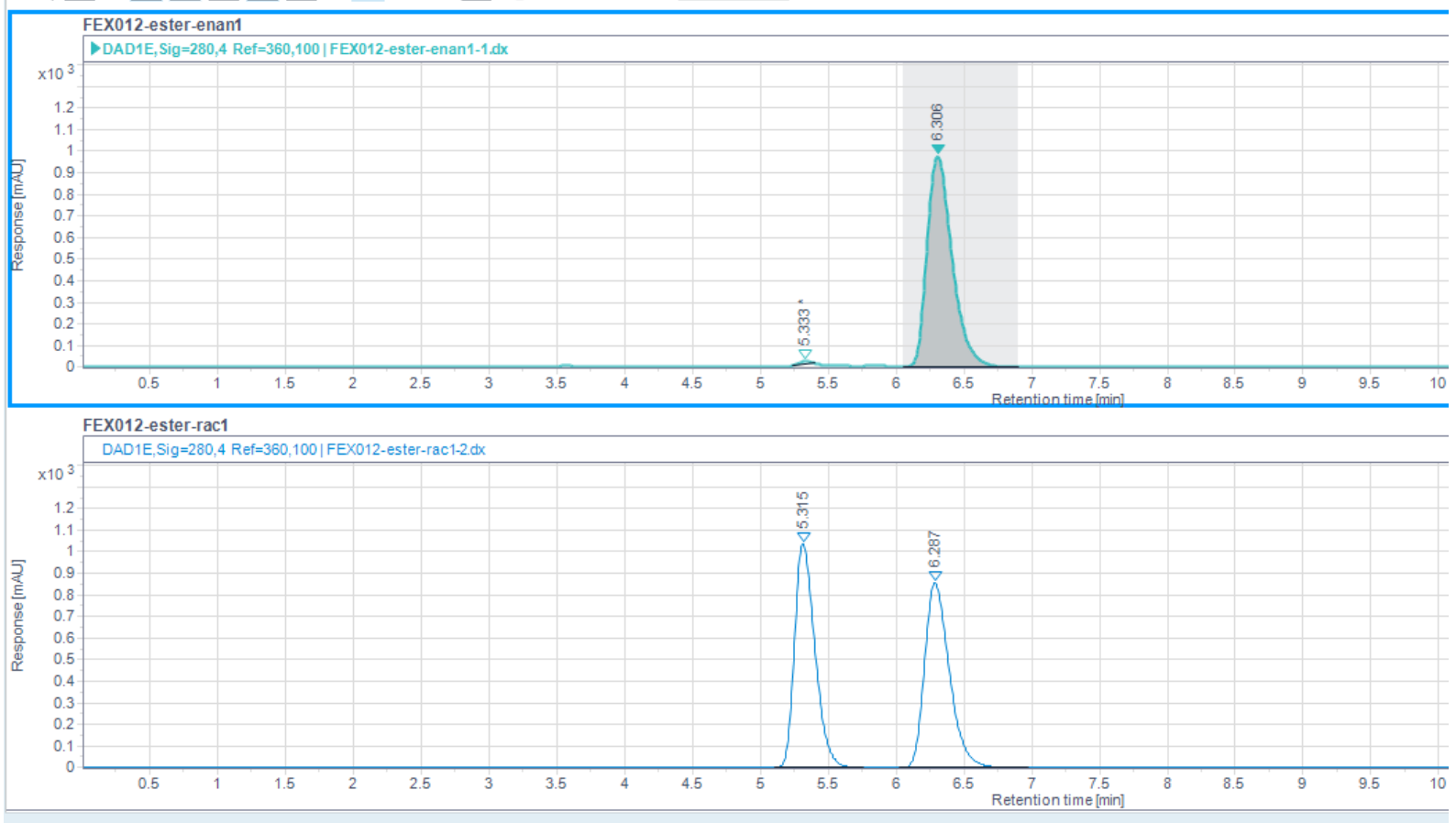

Injection Results

Peaks Summary

\begin{tabular}{|c|c|c|c|c|c|}
\hline \# & - Signal description & RT (min) & Area & Area\% & Height \\
\hline & 1 DAD1E,Sig $=280,4$ Ref $=360,100$ & 5.333 & 72.775 & 0.58 & 12.382 \\
\hline & 2 DAD1E,Sig $=280,4$ Ref $=360,100$ & 6.306 & 12569.608 & 99.42 & 974.429 \\
\hline
\end{tabular}

Figure 3: Racemate and enantioenriched sample of 5-OMe. 


\subsection{Carboxylic acid 5}

\section{Chromatograms}

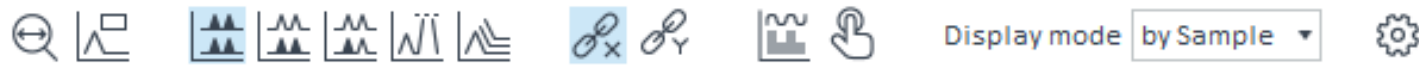

FEX-012-rac
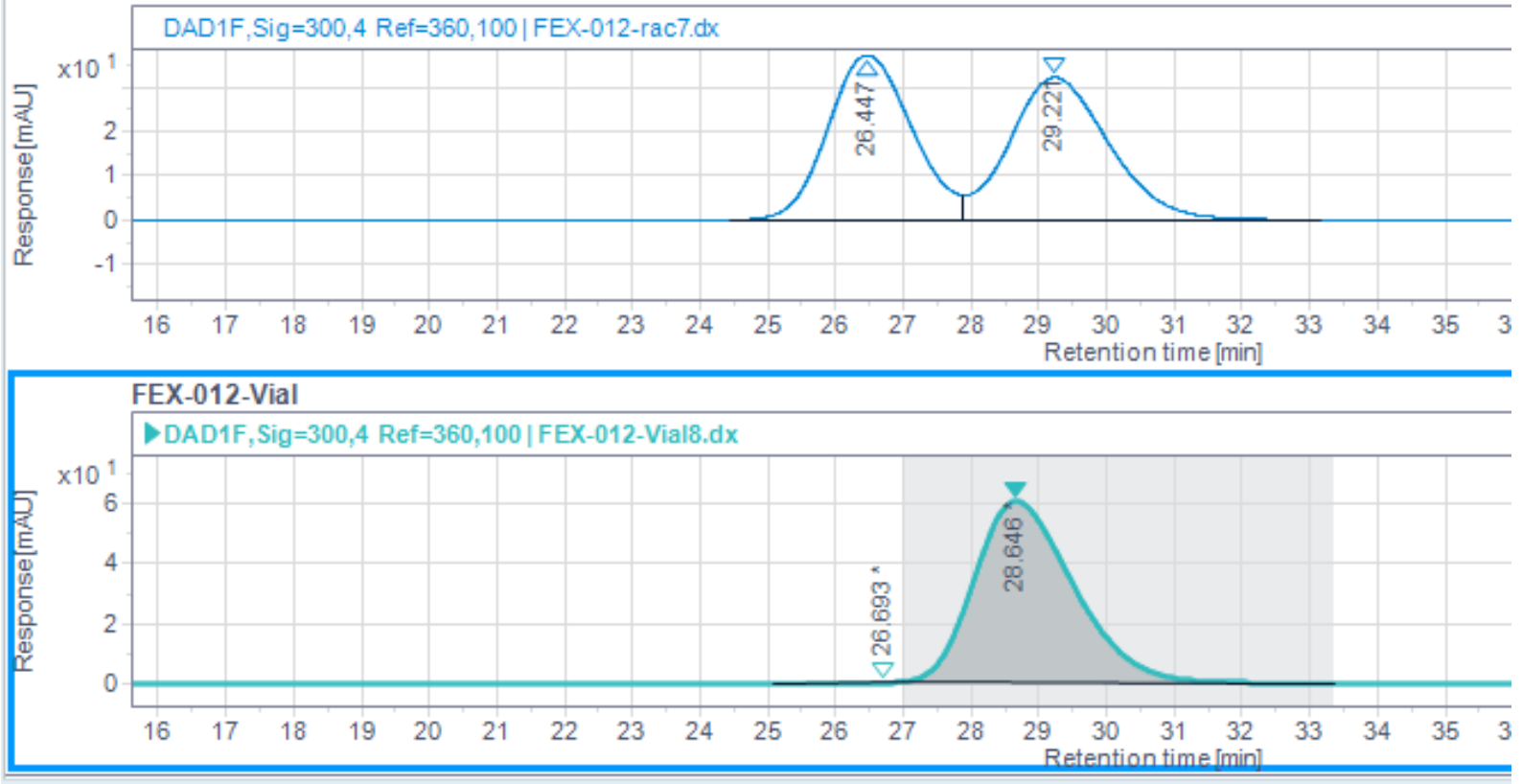

\section{Injection Results}

\begin{tabular}{|c|c|c|c|c|c|c|}
\hline & Peaks & Summary & & & & \\
\hline \multirow[t]{3}{*}{ ए+ } & \# & - Signal description & RT (min) & Area & Area\% & Height \\
\hline & & 1 DAD1F,Sig $=300,4$ Ref $=360,100$ & 26.693 & 18.119 & 0.294 & 0.296 \\
\hline & & 2 DAD1F,Sig $=300,4$ Ref $=360,100$ & 28.646 & 6149.732 & 99.706 & 60.249 \\
\hline
\end{tabular}

Figure 4: Racemate and enantioenriched sample of 5. 


\subsection{Galbulin (1)}

Chromatograms

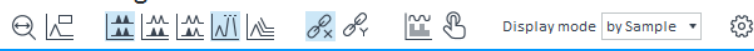
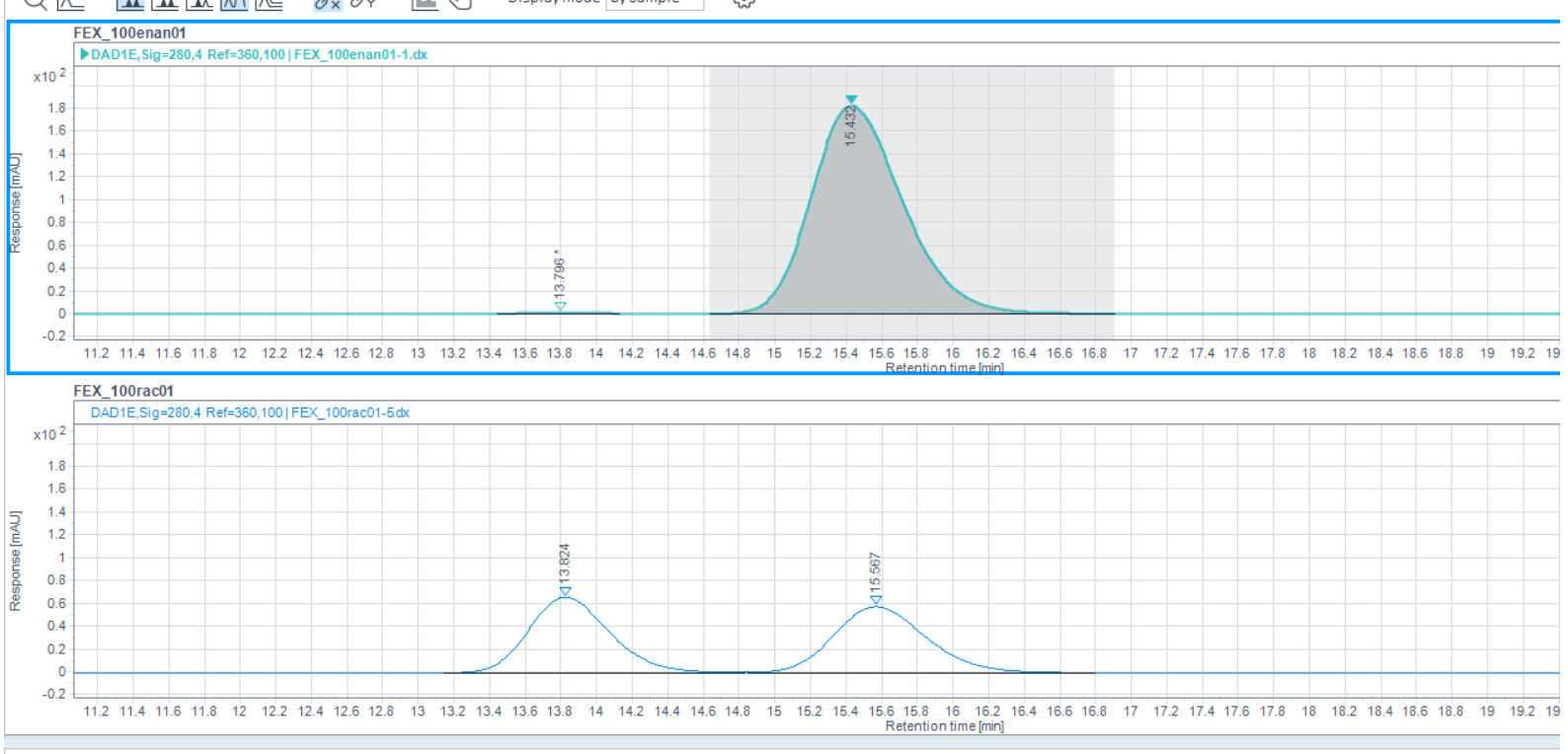

Injection Results

Peaks summary

T. \#. Signat destion

1 DAD1E,Sig $=280,4$ Ref $=360,100$

2 DADIE,Sig $=280,4$ Ref $=360,100$

\begin{tabular}{|c|r|r|r|}
\hline RT (min) & Area & \multicolumn{1}{l|}{ Area\% } & \multicolumn{1}{l|}{ Height } \\
\hline 13.796 & 26.470 & 0.40 & 1.137 \\
\hline 15.432 & 6555.793 & 99.60 & 182.186 \\
& & & \\
\end{tabular}

Figure 5: Racemate and enantioenriched of Galbulin (1).

\section{UV Spectrum}

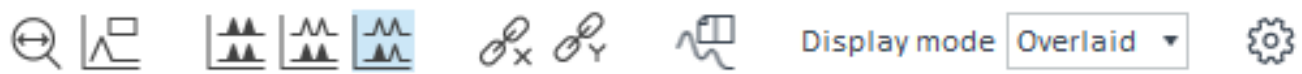

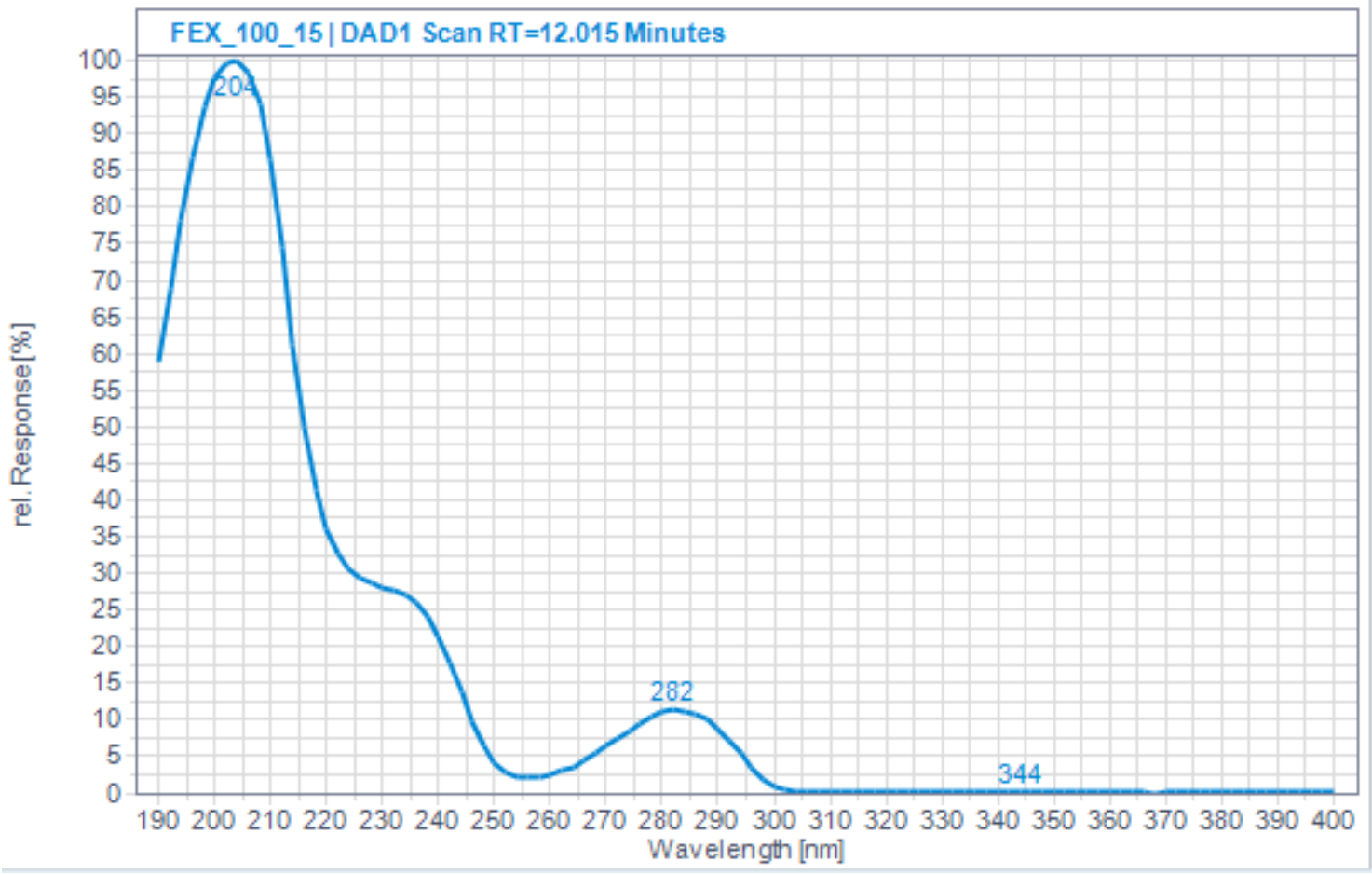

Figure 6: UV-absorption spectrum of Galbulin (1). 


\section{NMR data}

${ }^{1} \mathrm{H}_{\text {NMR }}\left(\mathrm{CDCl}_{\mathbf{3}}, \mathbf{3 0 0} \mathbf{M H z}\right)$ : 4,4-Diethoxybutyronitrile.
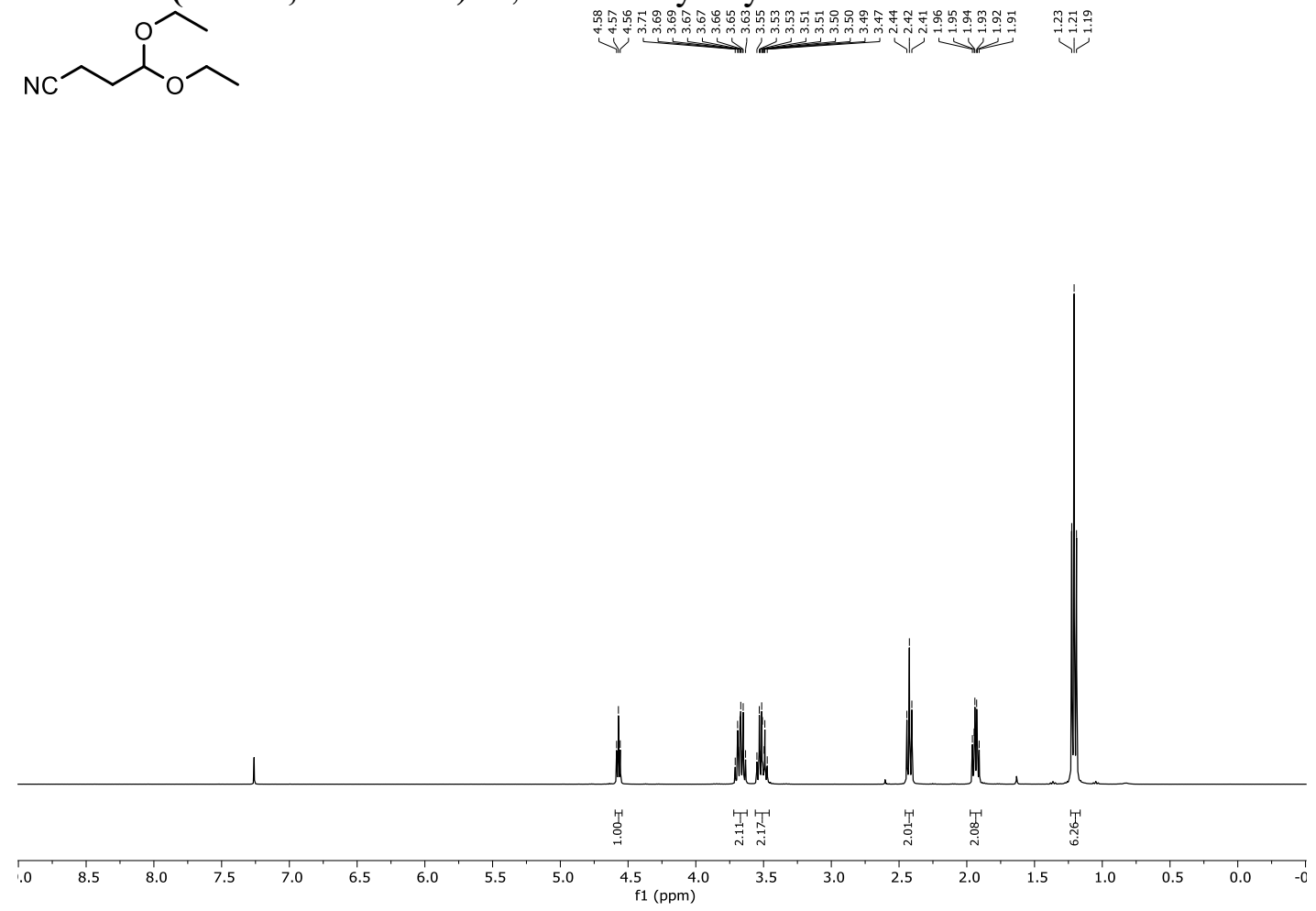

${ }^{13} \mathbf{C}\left\{{ }^{1} \mathbf{H}\right\}$ NMR $\left(\mathbf{C D C l}_{\mathbf{3}}, 75 \mathbf{M H z}\right)$ : 4,4-Diethoxybutyronitrile.<smiles>CCOC(CCC#N)OCC</smiles>
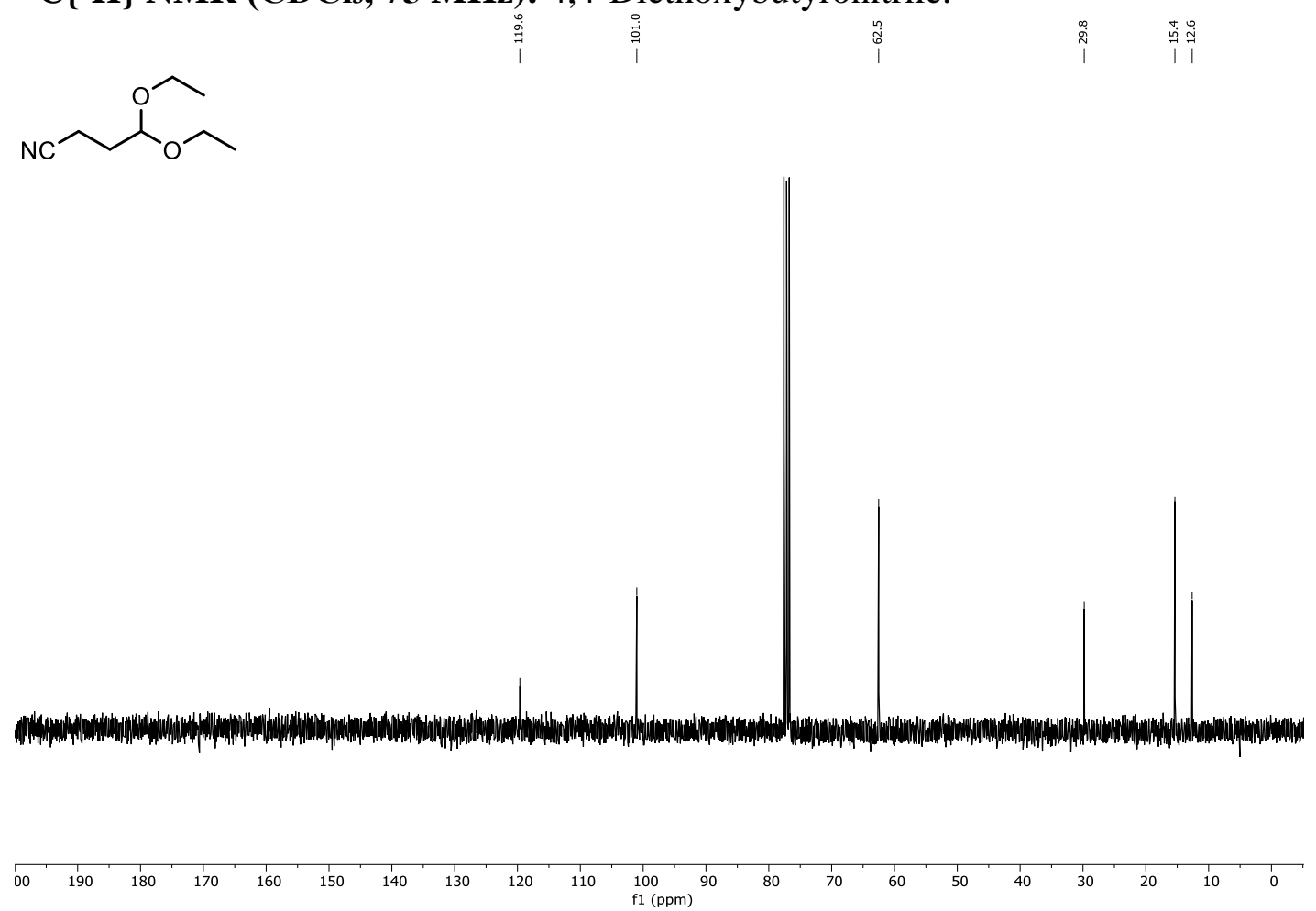
${ }^{1} \mathrm{H}$ NMR ( $\left.\mathrm{CDCl}_{3}, 300 \mathrm{MHz}\right)$ : 6,7-Dimethoxy-2-naphthonitrile (8).

Y

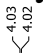<smiles>COc1cc2ccc(C#N)cc2cc1OC</smiles>

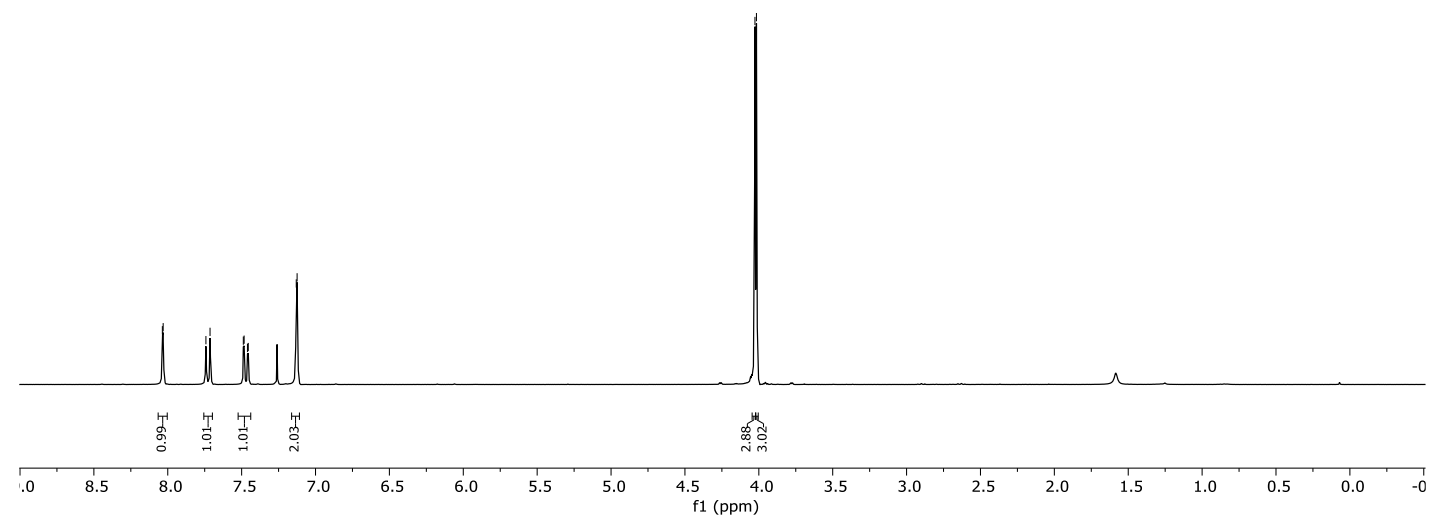

${ }^{13} \mathrm{C}\left\{{ }^{1} \mathrm{H}\right\}$ NMR ( $\left.\mathrm{CDCl}_{3}, 75 \mathrm{MHz}\right)$ : 6,7-Dimethoxy-2-naphthonitrile (8).<smiles>COc1cc2ccc(C#N)cc2cc1OC</smiles>
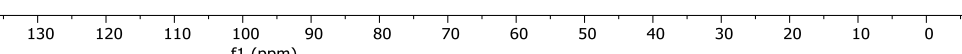
${ }^{1} \mathbf{H}$ NMR ( $\mathbf{C D C l}_{3}, 300$ MHz): (S)-2-(6,7-Dimethoxynaphthaline-2-yl)-4-isopropyl-4,5dihydrooxazole (6).
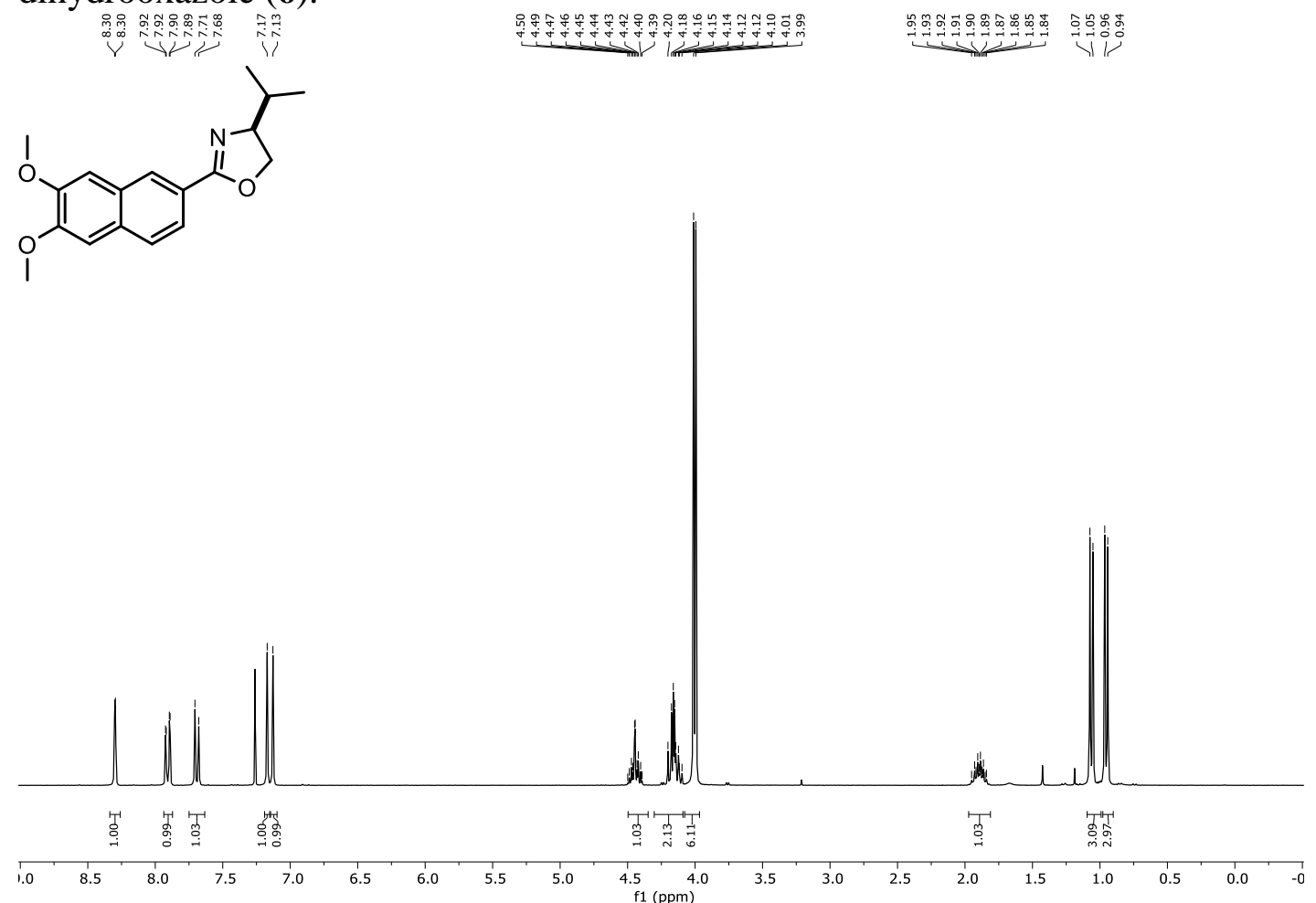

${ }^{13} \mathbf{C}\left\{{ }^{1} \mathrm{H}\right\}$ NMR $\left(\mathrm{CDCl}_{3}, 75 \mathrm{MHz}\right):(S)$-2-(6,7-Dimethoxynaphthaline-2-yl)-4-isopropyl-4,5dihydrooxazole $(\mathbf{6})$.
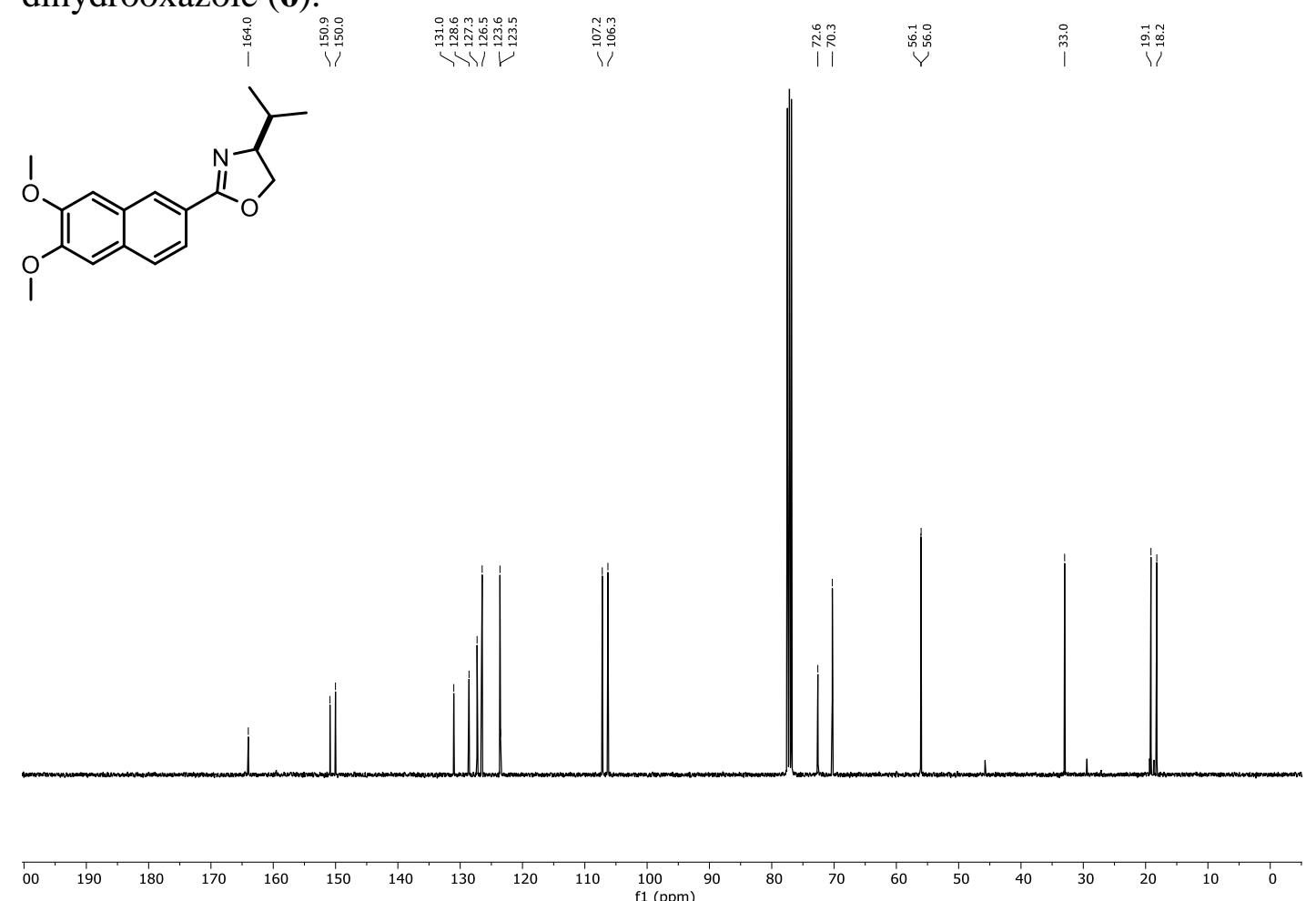
${ }^{1}$ H NMR (C6D6, 500 MHz): (S)-2-((1S,2S)-1-(Dimethyl(phenyl)silyl)-6,7-dimethoxy-2methyl-1,2-dihydronaphthalene-2-yl)-4-isopropyl-4,5-dihydrooxazole (9).
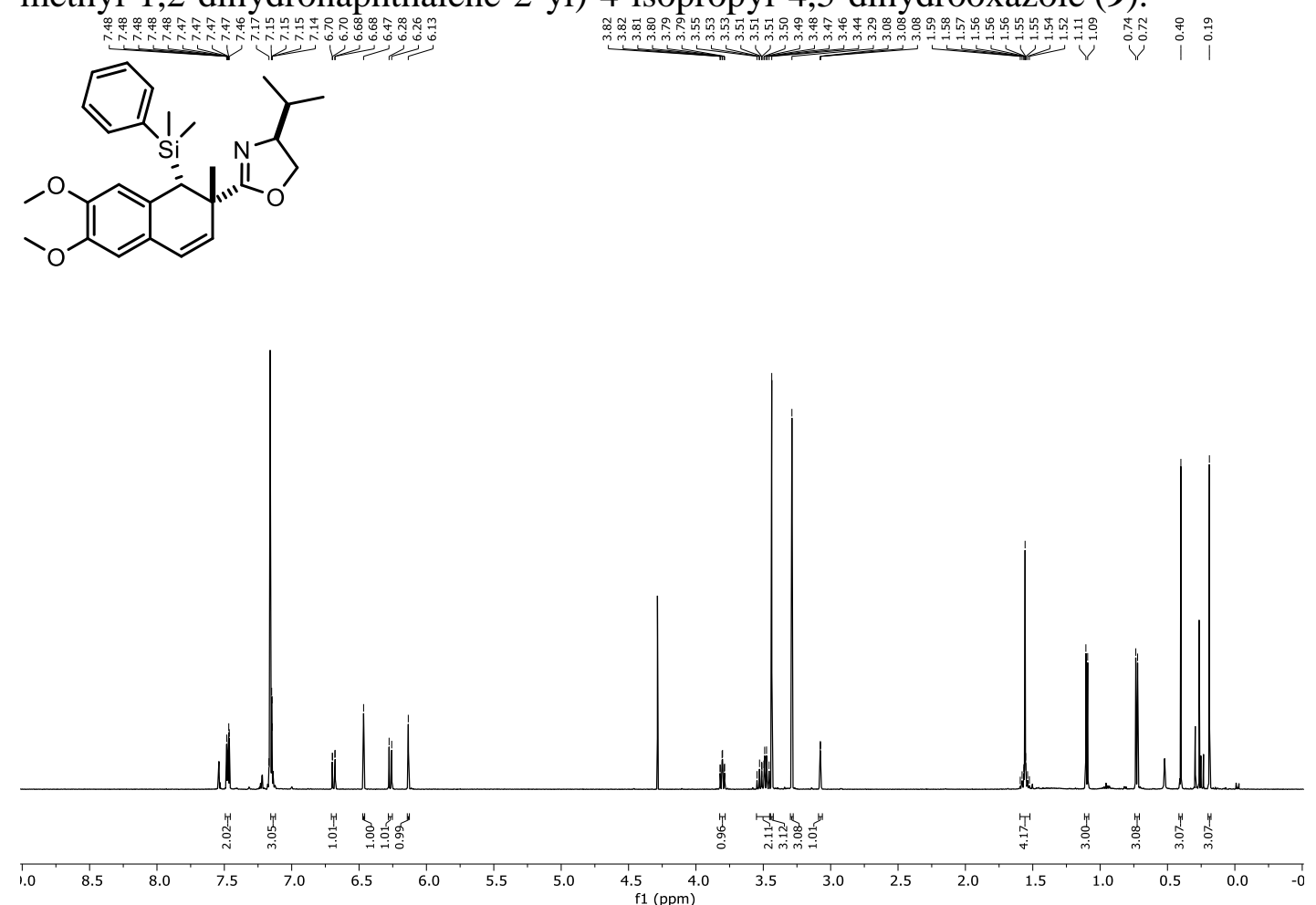

${ }^{13} \mathbf{C}\left\{{ }^{1} \mathbf{H}\right\}$ NMR (C6 $\mathbf{D}_{6}, 125$ MHz): $(S)-2-((1 S, 2 S)$-1-(Dimethyl(phenyl)silyl)-6,7-dimethoxy-2methyl-1,2-dihydronaphthalene-2-yl)-4-isopropyl-4,5-dihydrooxazole (9).

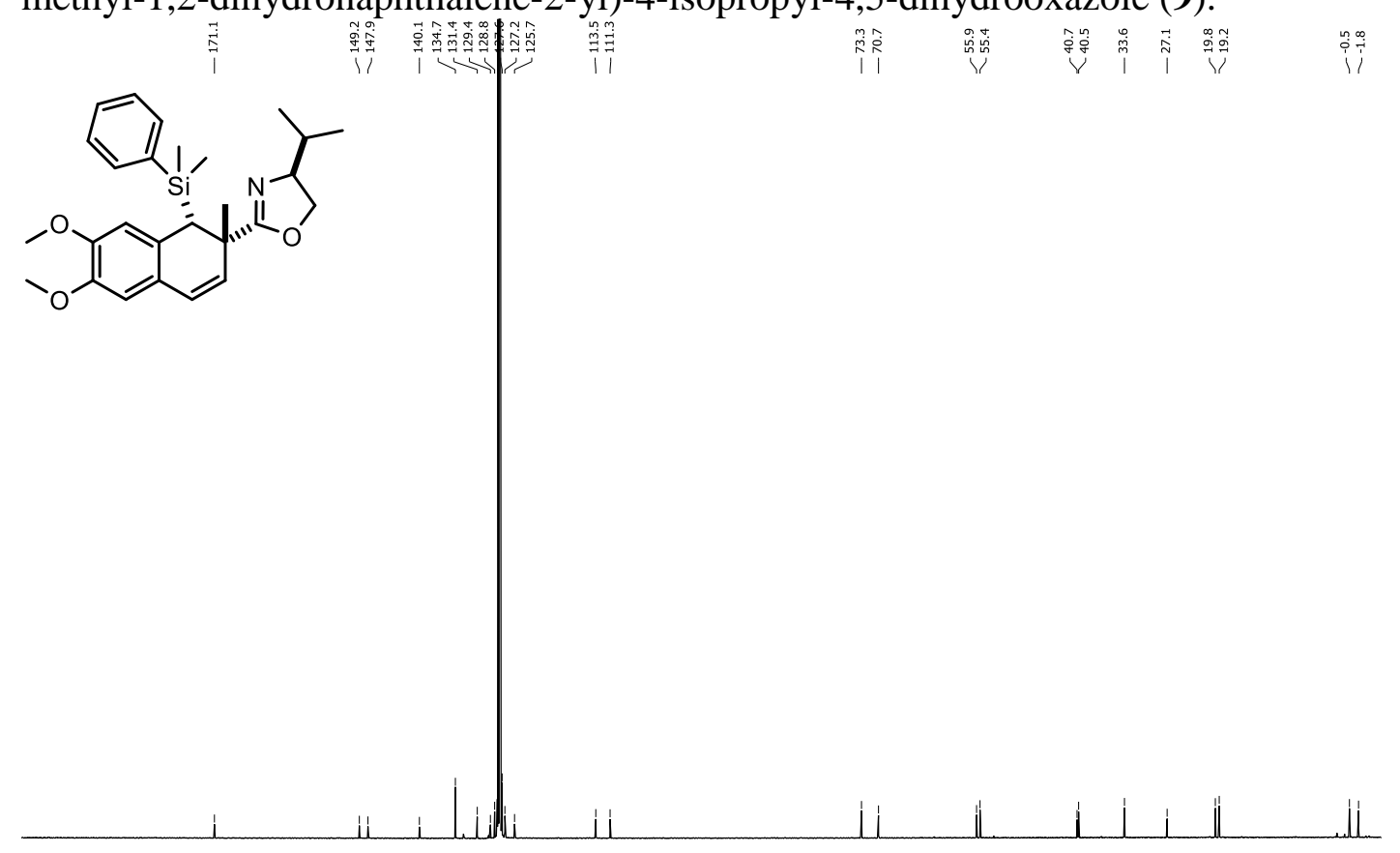

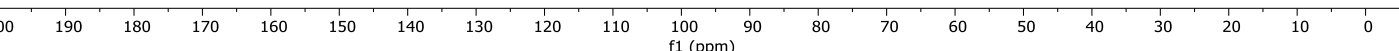


${ }^{1} \mathbf{H}$ NMR ( $\mathbf{C D}_{2} \mathbf{C l}_{2}, 500$ MHz): (S)-6,7-Dimethoxy-2-methyl-1,2-dihydronaphthalene-2carboxylic acid (5).<smiles>C=CC1(C(=O)O)Cc2cc(OC)c(OC)cc2C1</smiles>

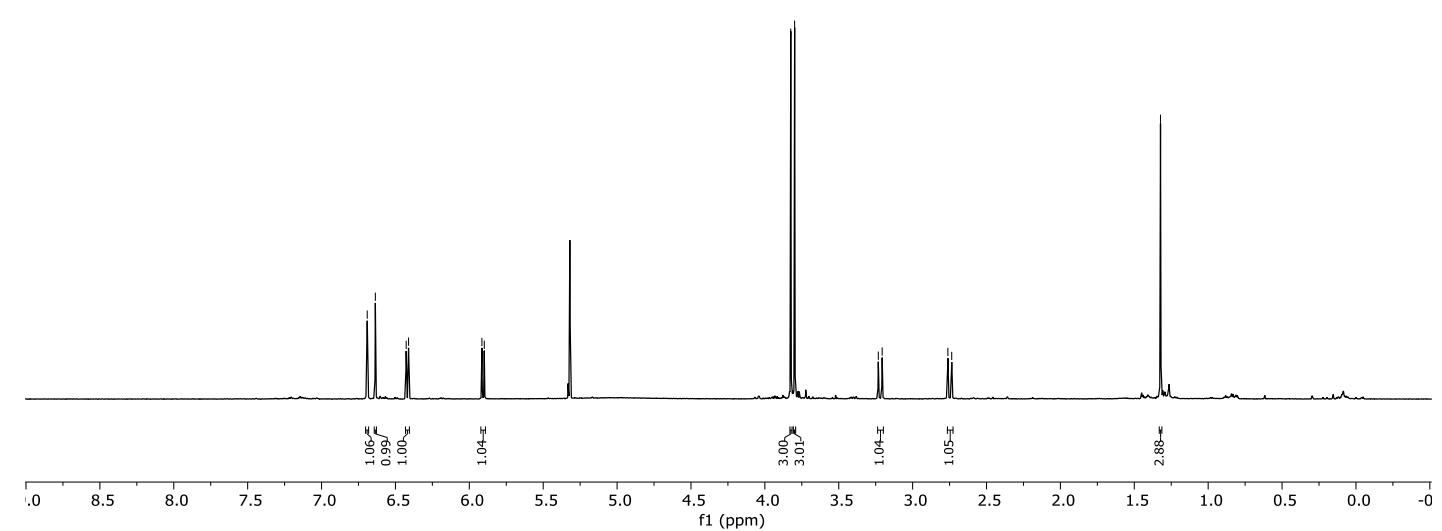

${ }^{13} \mathbf{C}\left\{{ }^{1} \mathbf{H}\right\}$ NMR ( $\mathbf{C D}_{2} \mathbf{C l}_{2}, 125$ MHz): (S)-6,7-Dimethoxy-2-methyl-1,2-dihydronaphthalene-2carboxylic acid (5).
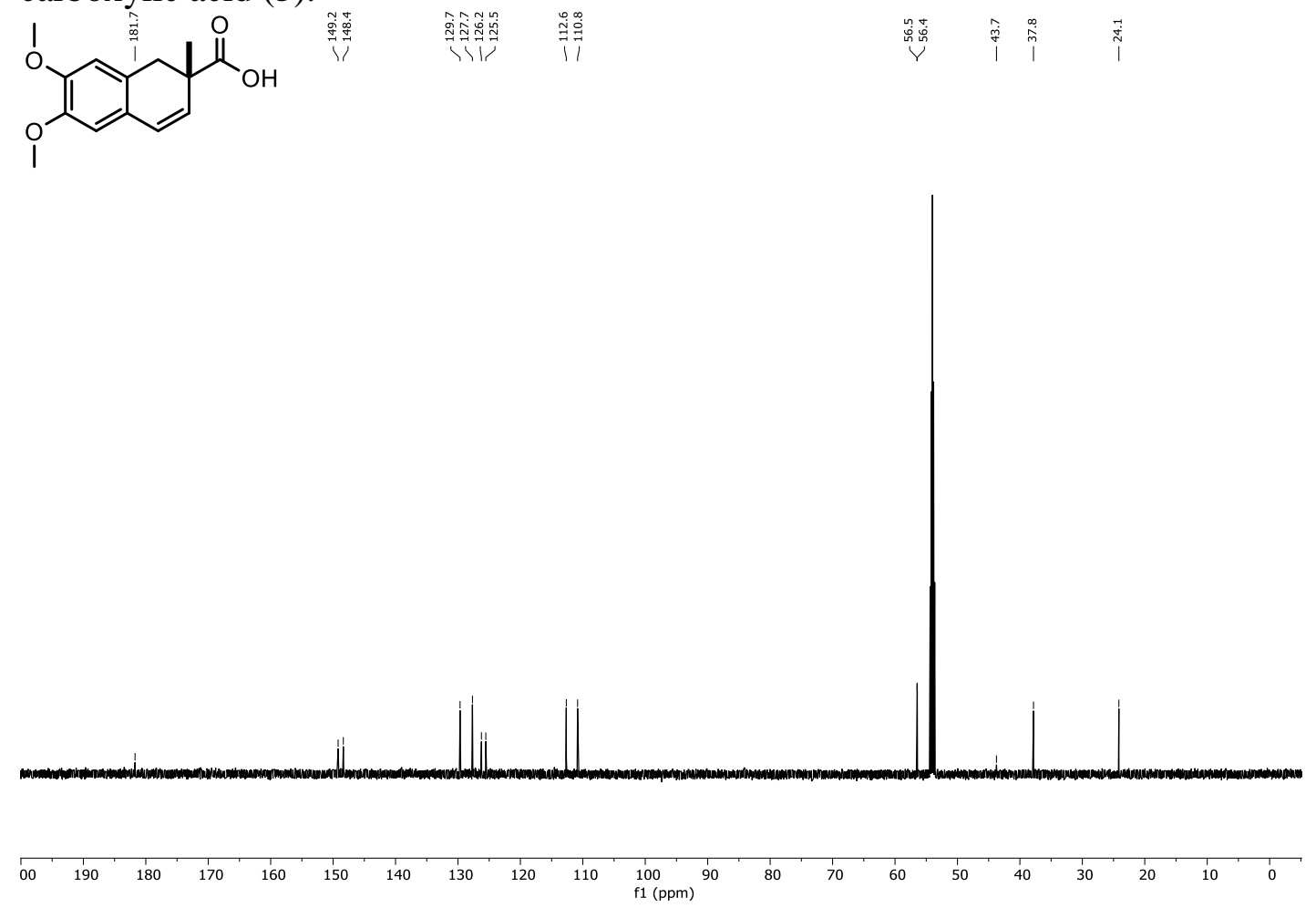
${ }^{1} \mathbf{H}$ NMR (CDCl, 600 MHz): (S)-Methyl 6,7-Dimethoxy-2-methyl-1,2-dihydronaphthalene2-carboxylate (5-OMe).
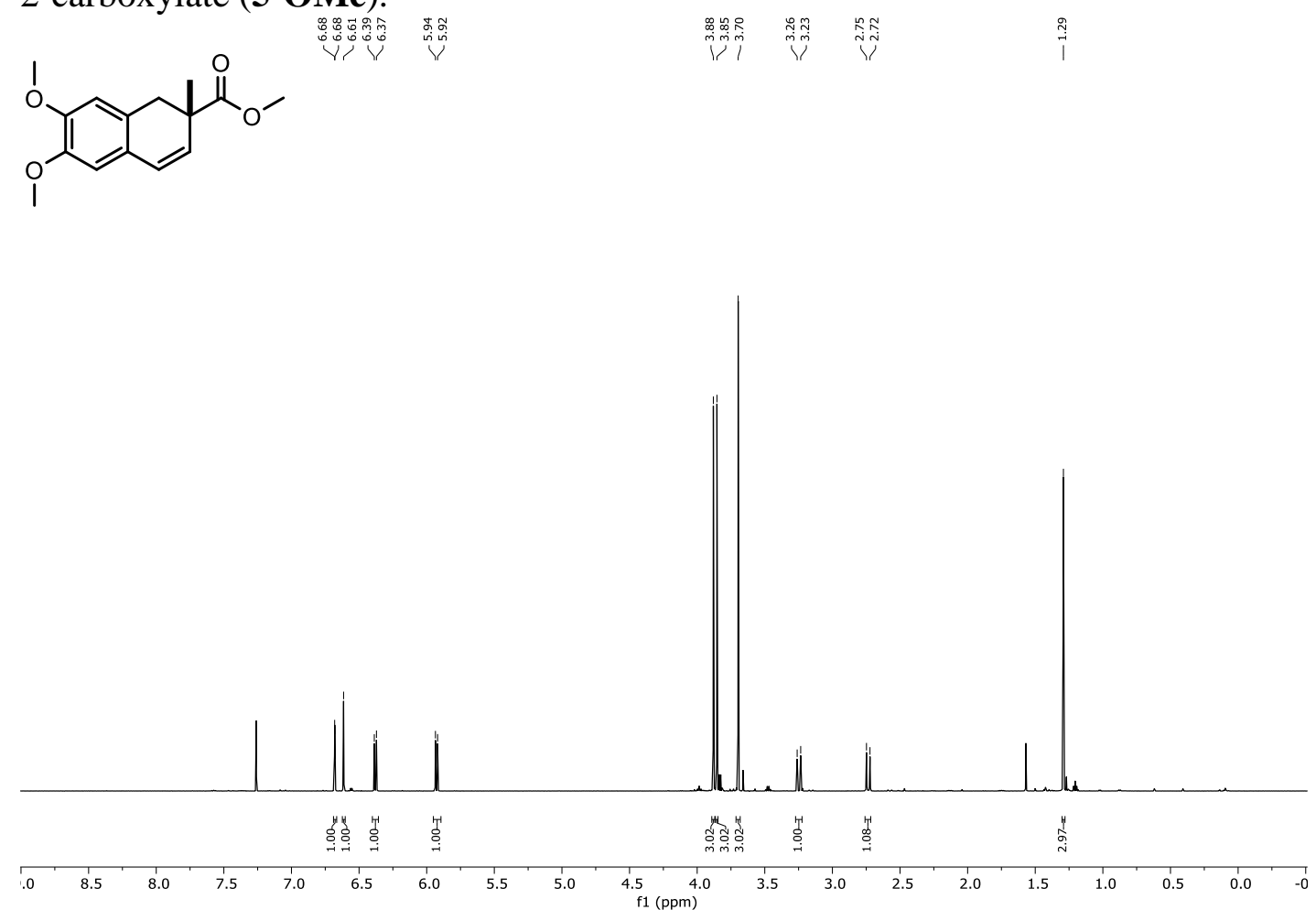

${ }^{13} \mathbf{C}\left\{{ }^{1} \mathbf{H}\right\}$ NMR (CDCl 3,150 MHz): (S)-Methyl 6,7-Dimethoxy-2-methyl-1,2dihydronaphthalene-2-carboxylate (5-OMe).<smiles>COC(=O)C12C=Cc3cc(OC)c(OC)cc3C1(C)C2</smiles> 
${ }^{1} \mathrm{H}$ NMR ( $\left.\mathrm{C}_{6} \mathrm{D}_{6}, 600 \mathrm{MHz}\right)$ : Boronic ester 17 (major and minor isomer picked).

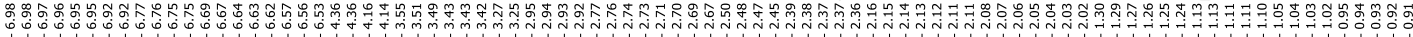

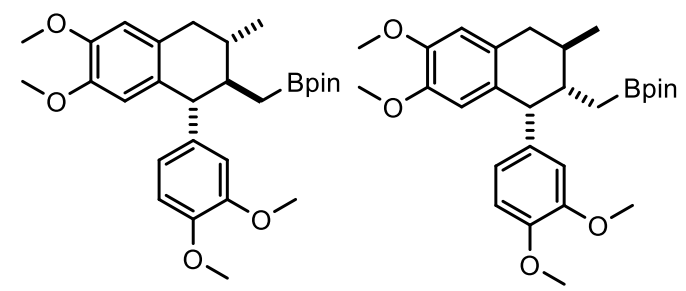

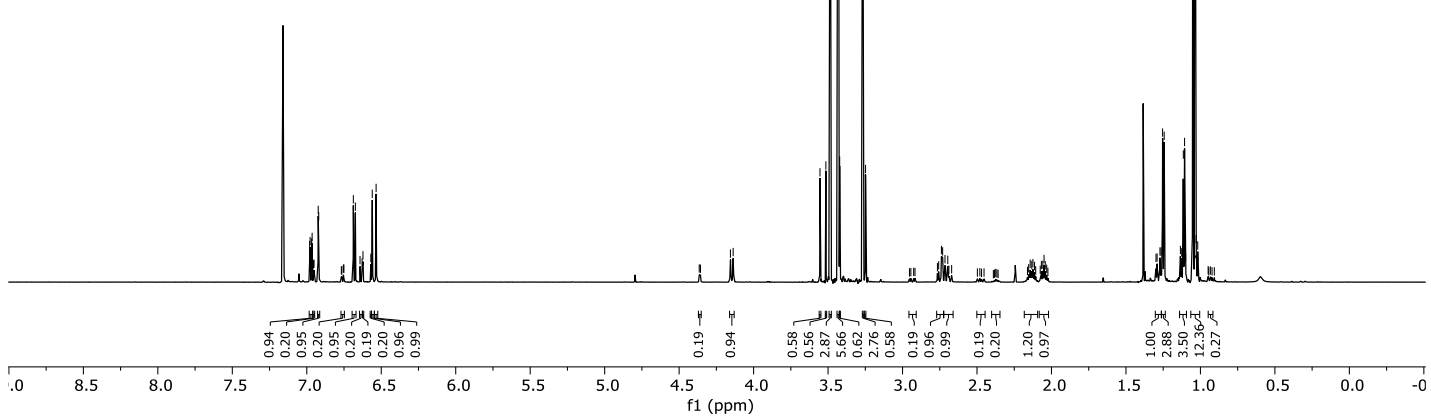

${ }^{13} \mathrm{C}\left\{{ }^{1} \mathrm{H}\right\}$ NMR (C6 $\left.\mathrm{D}_{6}, 150 \mathrm{MHz}\right)$ : Boronic ester 17 (major isomer picked).
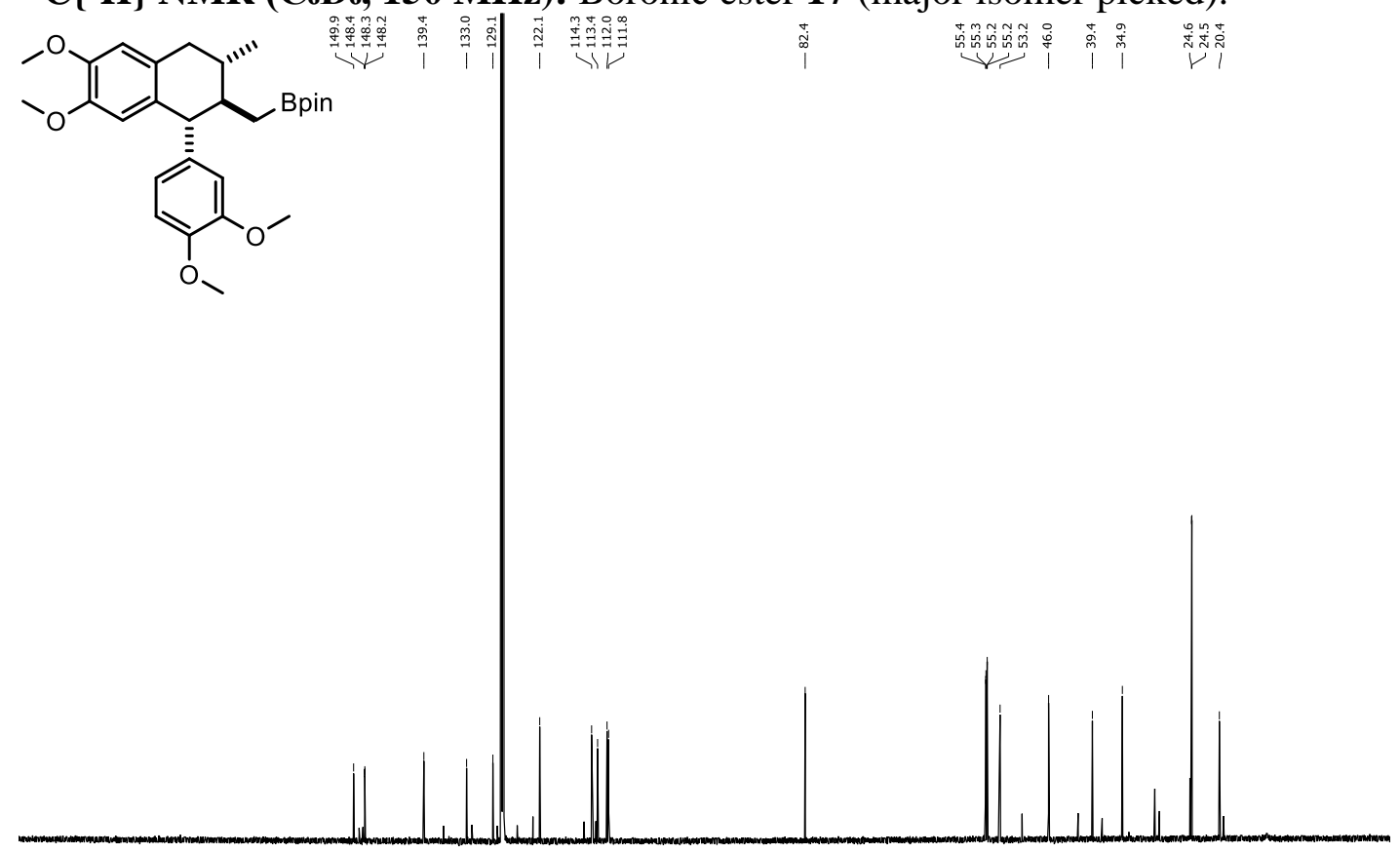

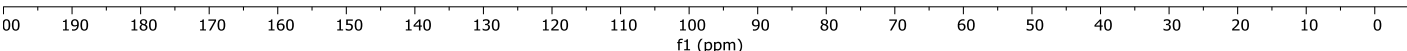


${ }^{13} \mathrm{C}\left\{{ }^{1} \mathrm{H}\right\}$ NMR $\left(\mathrm{C}_{6} \mathrm{D}_{6}, 150 \mathrm{MHz}\right)$ : Boronic ester 17 (minor isomer picked).
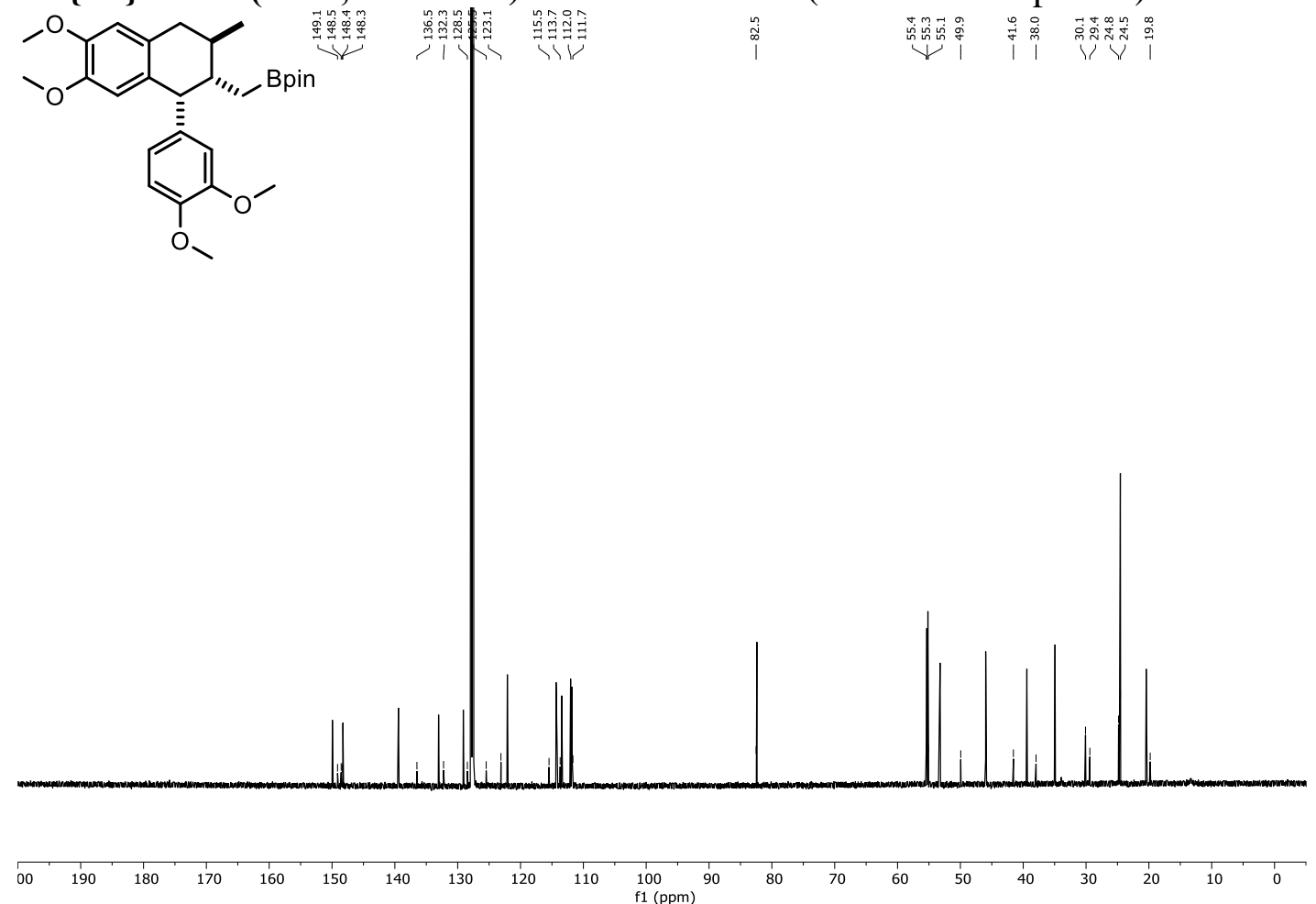

${ }^{1} \mathrm{H}$ NMR (C6 6 6, 500 MHz): Galbulin (1).

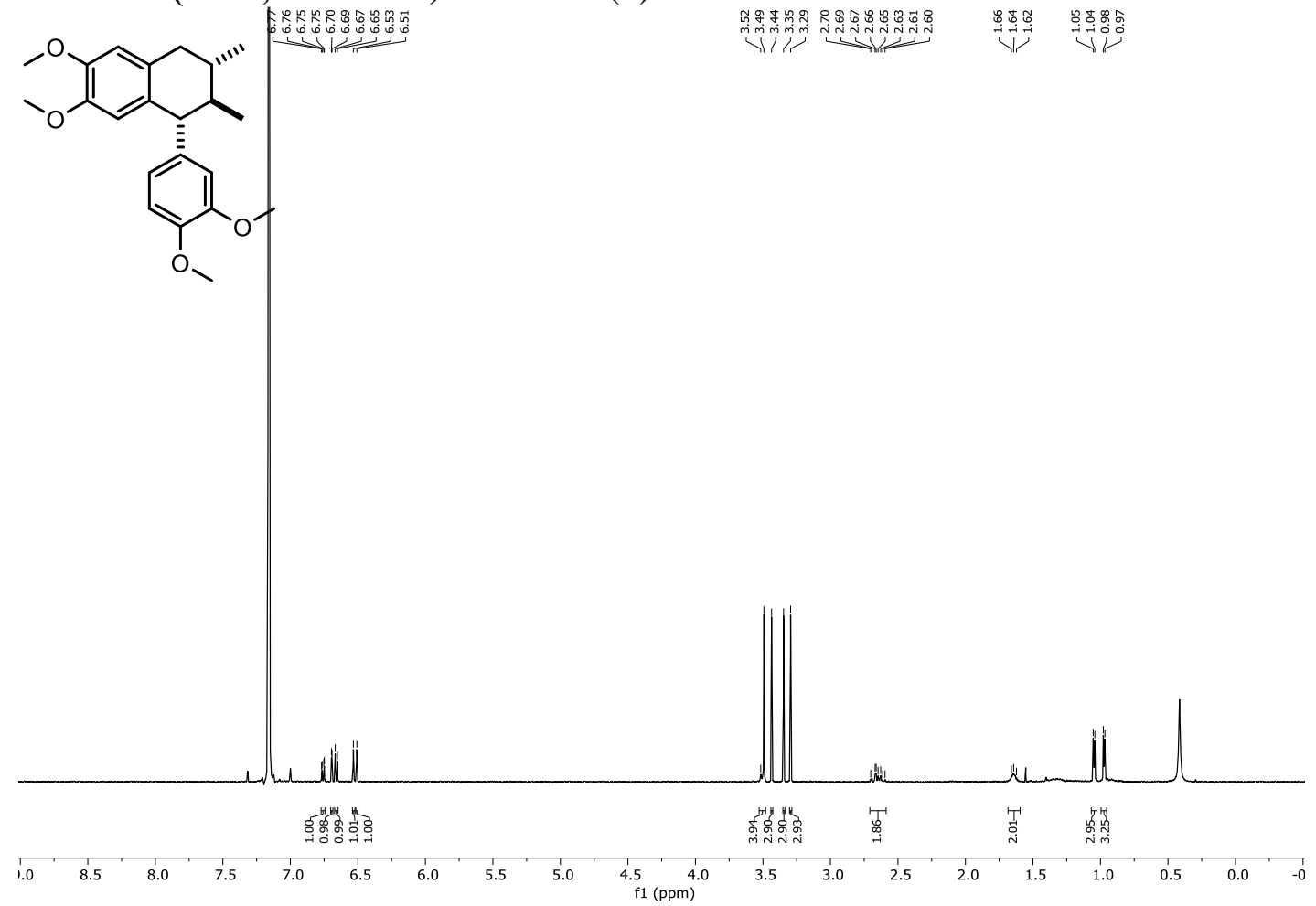




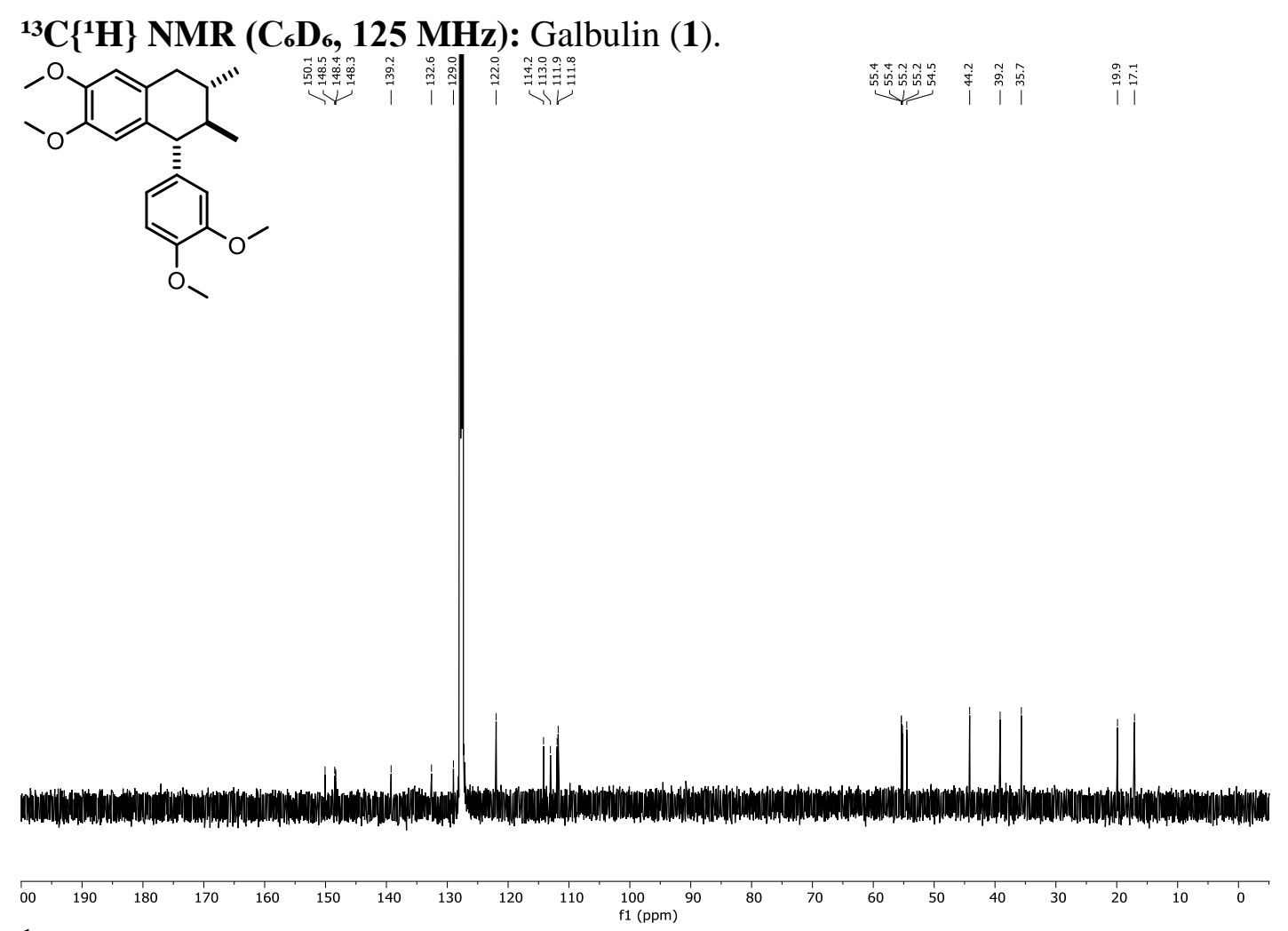

${ }^{1} \mathbf{H}$ NMR (CDCl 3,300 MHz): Rac-(S)-4-(tert-butyl)-2-(naphthalen-2-yl)-4,5-dihydrooxazole (22).

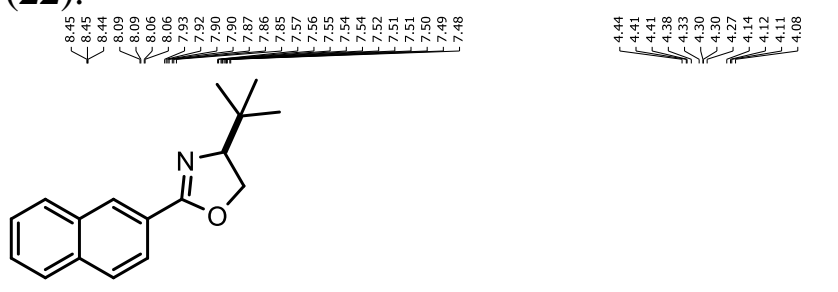

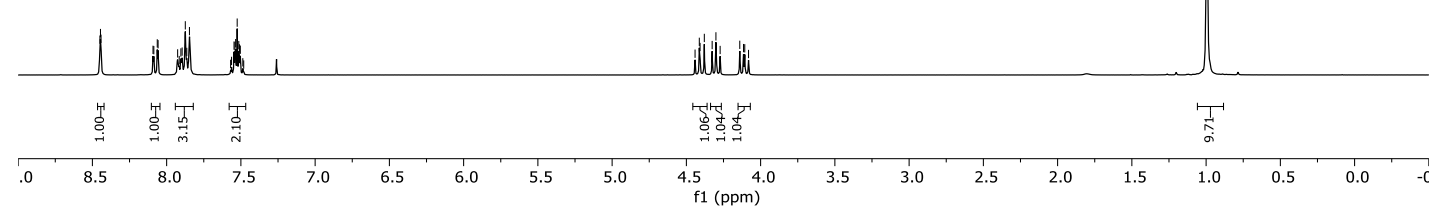


${ }^{13} \mathbf{C}\left\{{ }^{1} \mathbf{H}\right\}$ NMR $\left(\mathbf{C D C l}_{3}, 75\right.$ MHz): Rac-(S)-4-(tert-butyl)-2-(naphthalen-2-yl)-4,5dihydrooxazole (22).
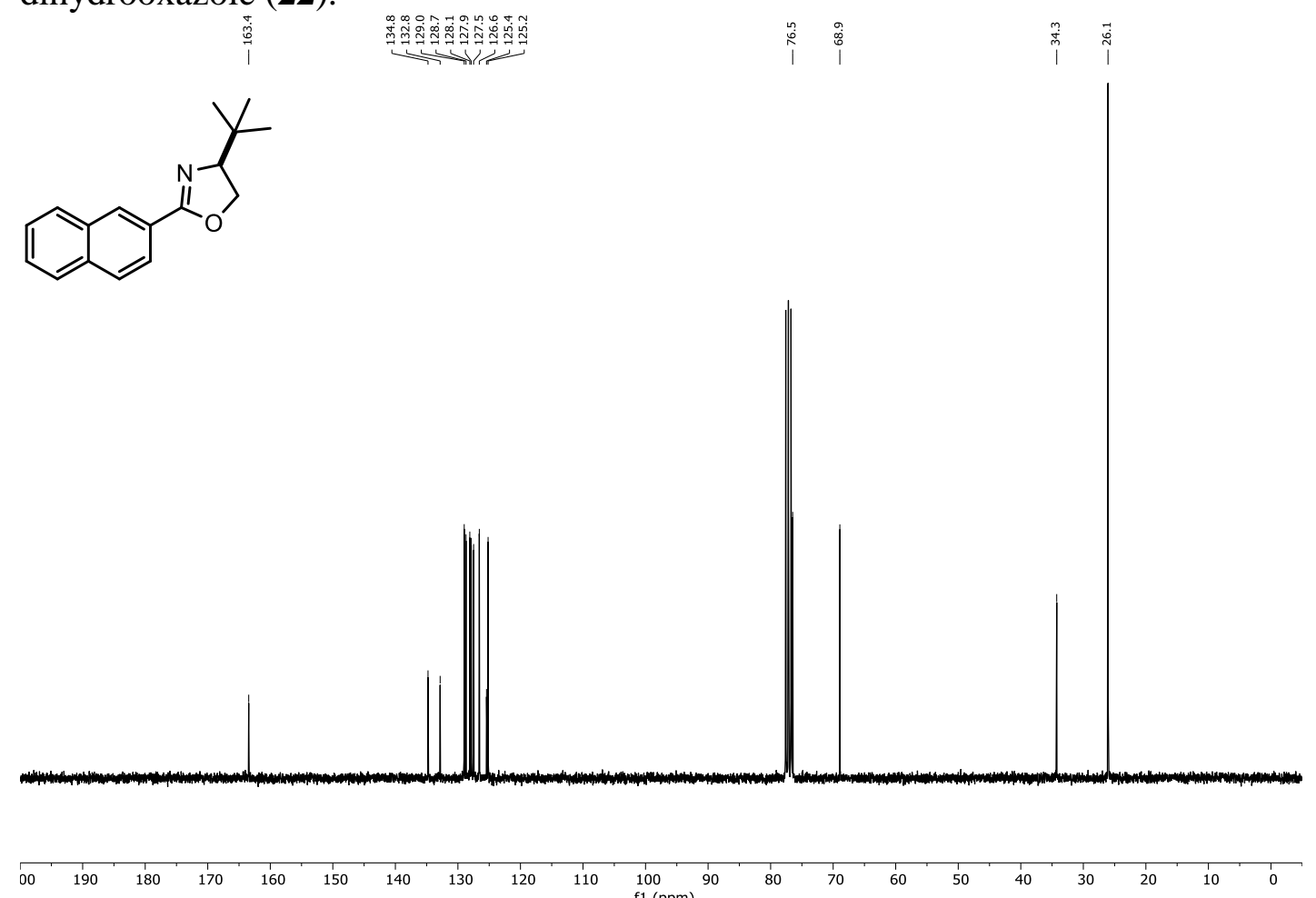

${ }^{1} \mathrm{H}$ NMR ( $\left.\mathrm{CDCl}_{3}, 300 \mathrm{MHz}\right)$ : Oxazoline 23.

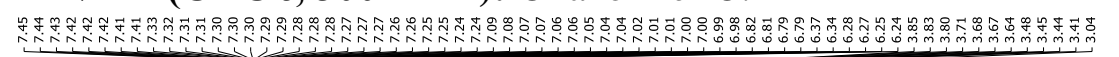<smiles>CC(C)(C)C1COC(C2(C)C=Cc3ccccc3[C@H]2[Si](C)(C)[PH3])=N1</smiles>

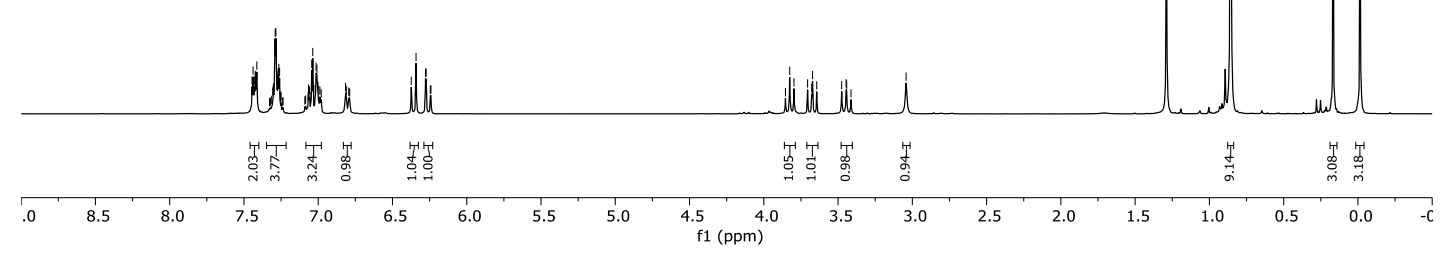


${ }^{13} \mathrm{C}\left\{{ }^{1} \mathrm{H}\right\}$ NMR $\left(\mathrm{CDCl}_{3}, 75 \mathrm{MHz}\right)$ : Oxazoline 23.

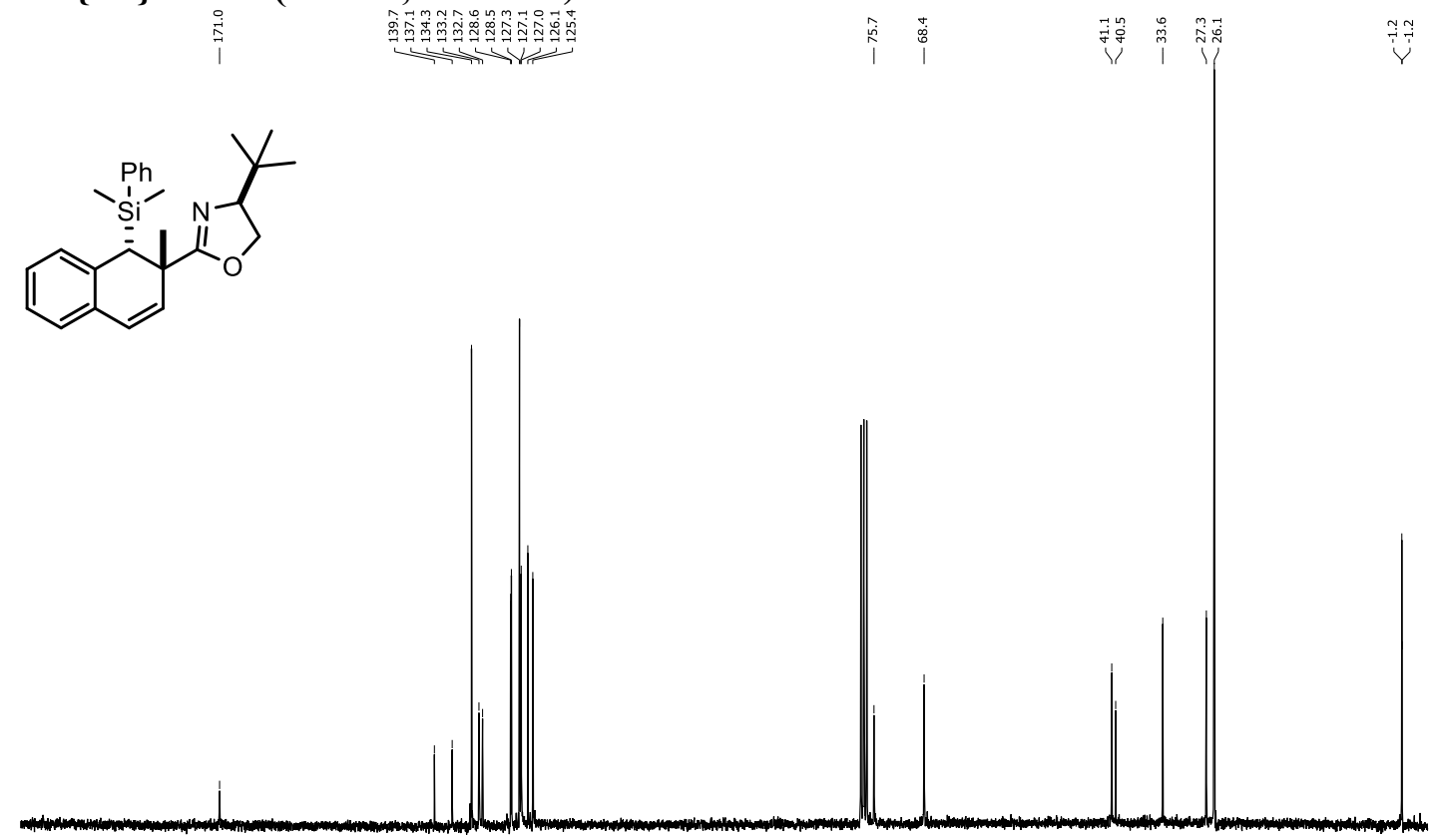

${ }^{1} \mathrm{H}$ NMR ( $\left.\mathrm{CDCl}_{3}, 300 \mathrm{MHz}\right)$ : Oxazoline 24.

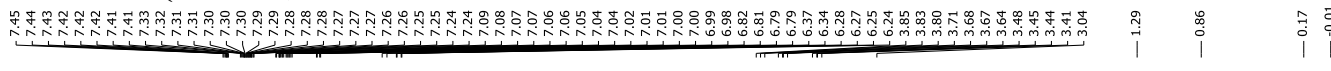<smiles>CC1(C2=NC(C(C)(C)C)CO2)C=Cc2ccccc2C1</smiles>

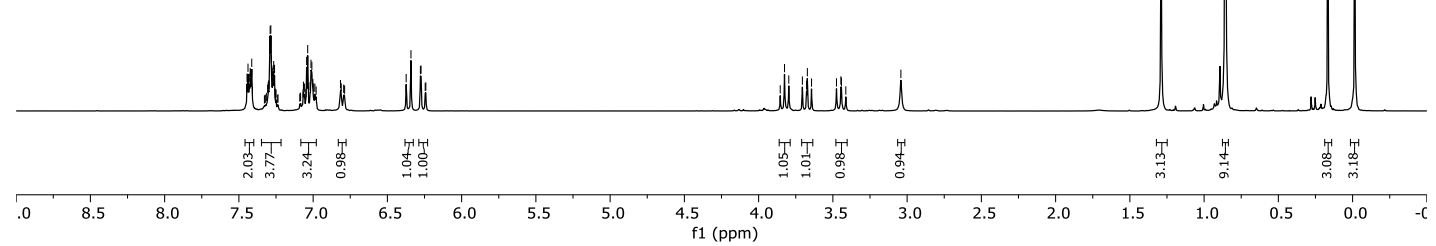


${ }^{13} \mathrm{C}\left\{{ }^{1} \mathrm{H}\right\}$ NMR $\left(\mathrm{CDCl}_{3}, 75 \mathrm{MHz}\right)$ : Oxazoline 24.

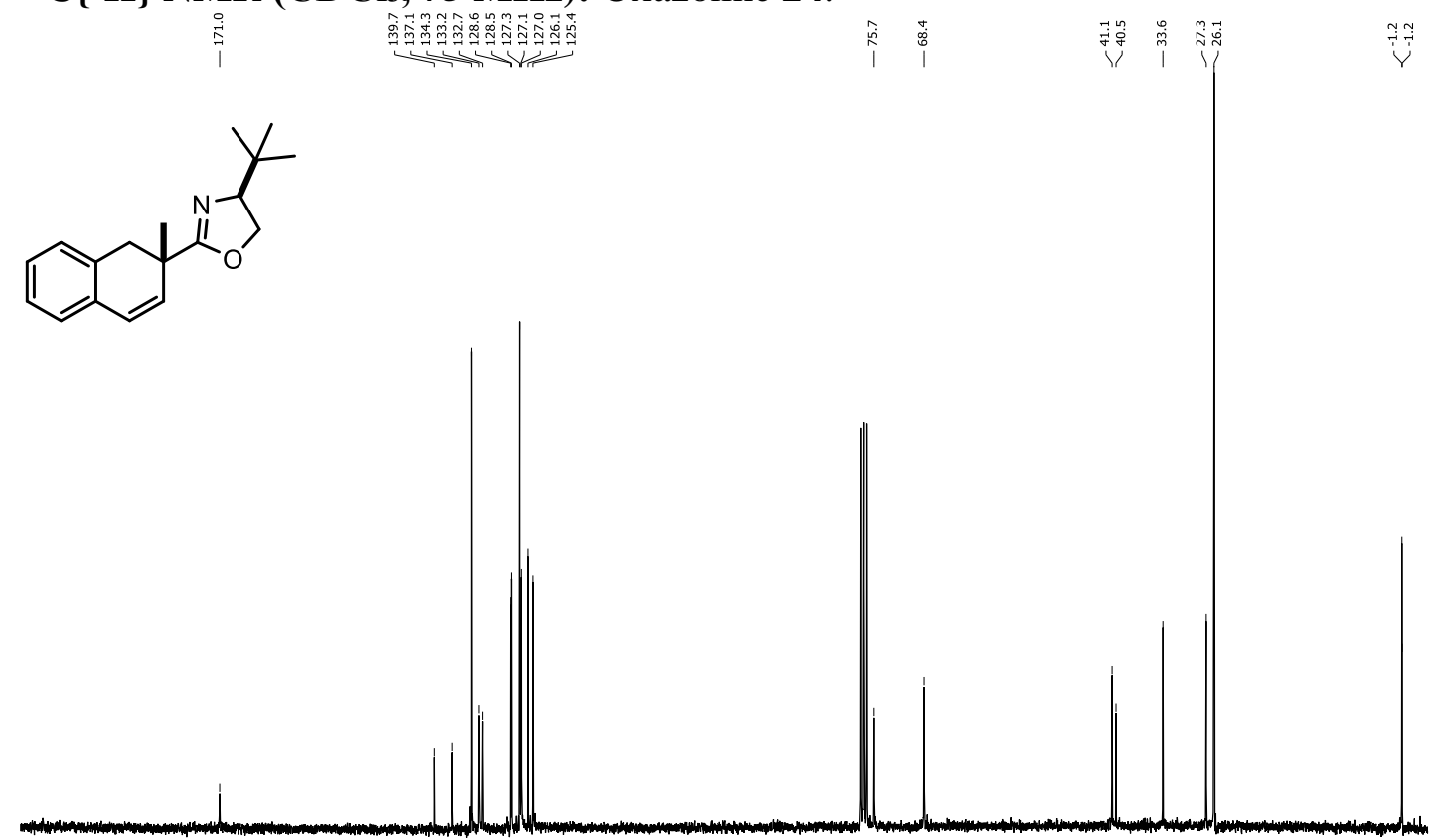

${ }^{1} \mathrm{H}$ NMR (CDCl $\left.3,300 \mathrm{MHz}\right)$ : Acid 20.

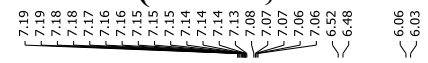<smiles>CC1(C(=O)O)C=Cc2ccccc2C1</smiles>

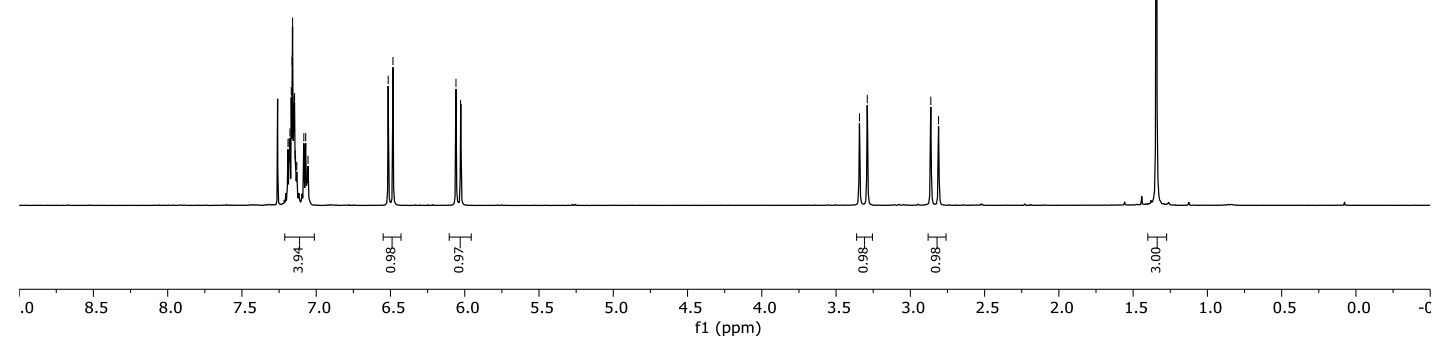


${ }^{1} \mathrm{H}$ NMR ( $\left.\mathrm{CDCl}_{3}, 75 \mathrm{MHz}\right)$ : Acid 20.
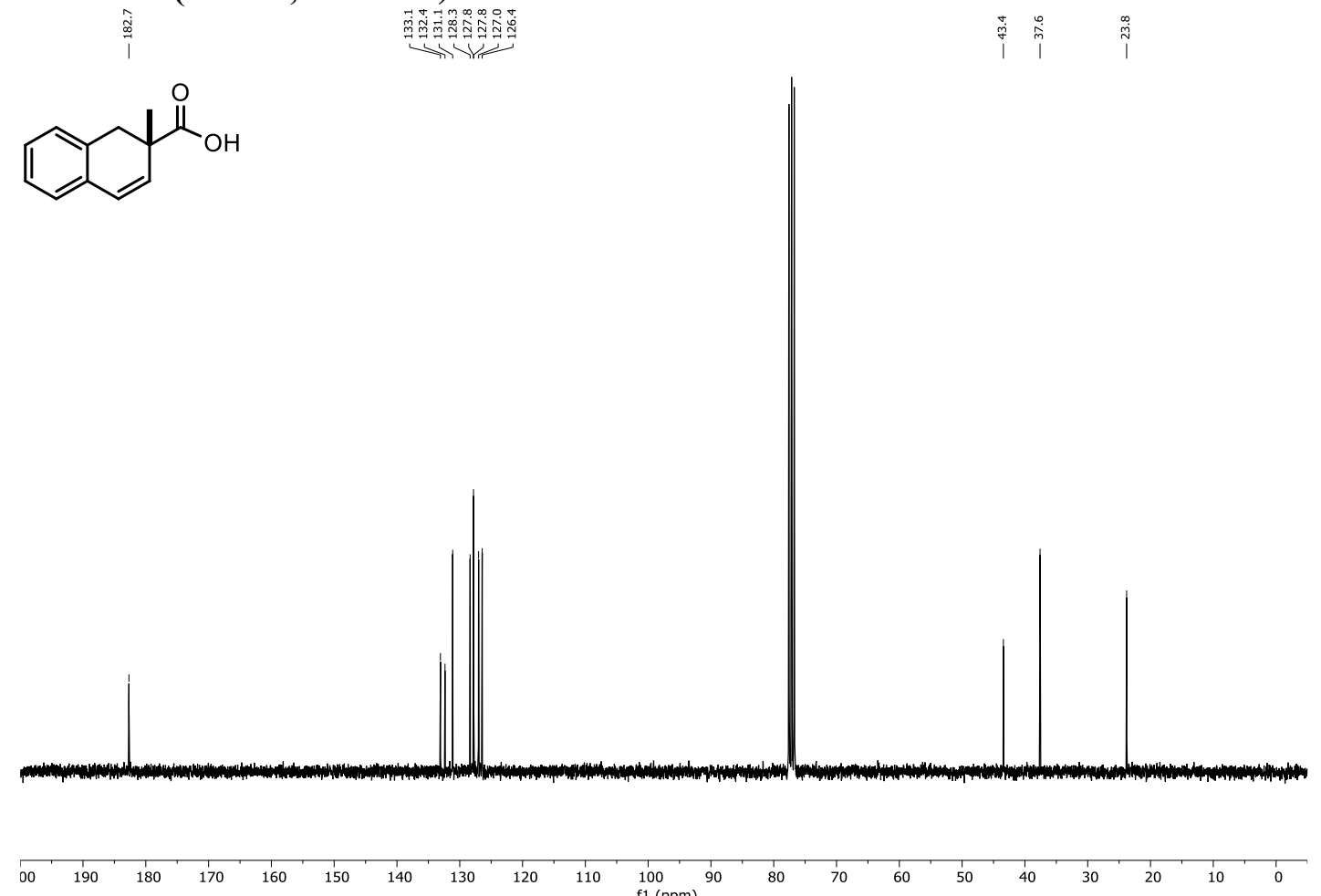

${ }^{1} \mathrm{H}$ NMR ( $\left.\mathrm{CD}_{2} \mathrm{Cl}_{2}, 500 \mathrm{MHz}\right)$ : Boronic ester 25 (major isomer picked).

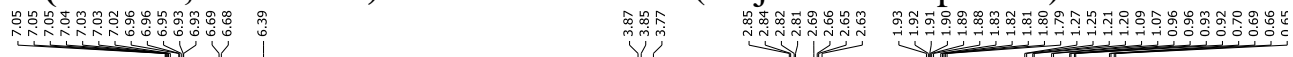<smiles>COc1cc([C@H]2c3ccccc3C[C@@H](C)[C@H]2COC(C)(C)C(C)(C)C)cc(OC)c1OC</smiles>

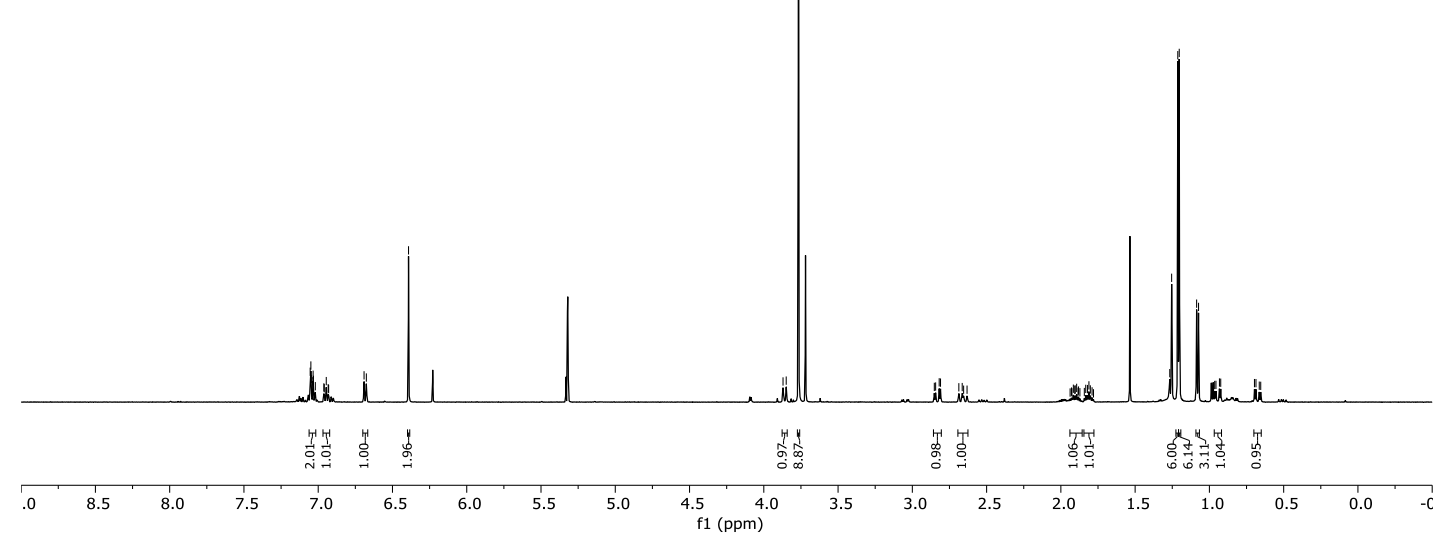


${ }^{1} \mathrm{H}$ NMR $\left(\mathrm{CD}_{2} \mathrm{Cl}_{2}, 500 \mathrm{MHz}\right)$ : Boronic ester 25 (minor isomer picked).

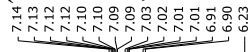<smiles>COc1cc(C2c3ccccc3C[C@H](C)C2COP(OC(C)(C)C)C(C)(C)C)cc(OC)c1OC</smiles>

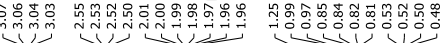

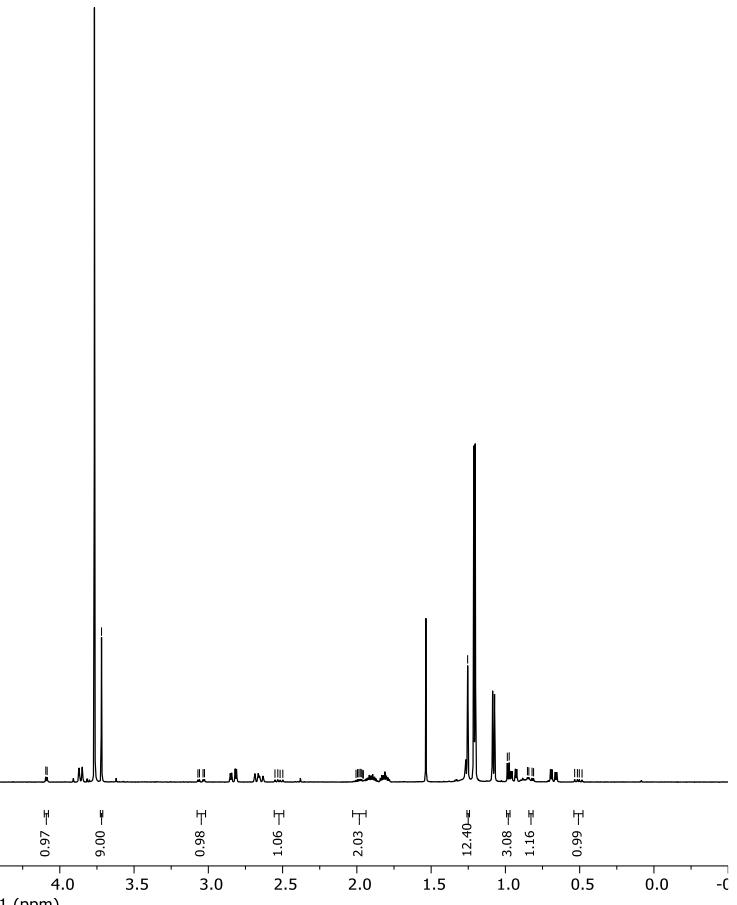

${ }^{13} \mathrm{C}\left\{{ }^{1} \mathrm{H}\right\}$ NMR $\left(\mathrm{CD}_{2} \mathrm{Cl}_{2}, 125 \mathrm{MHz}\right)$ : Boronic ester 25 (major isomer picked).

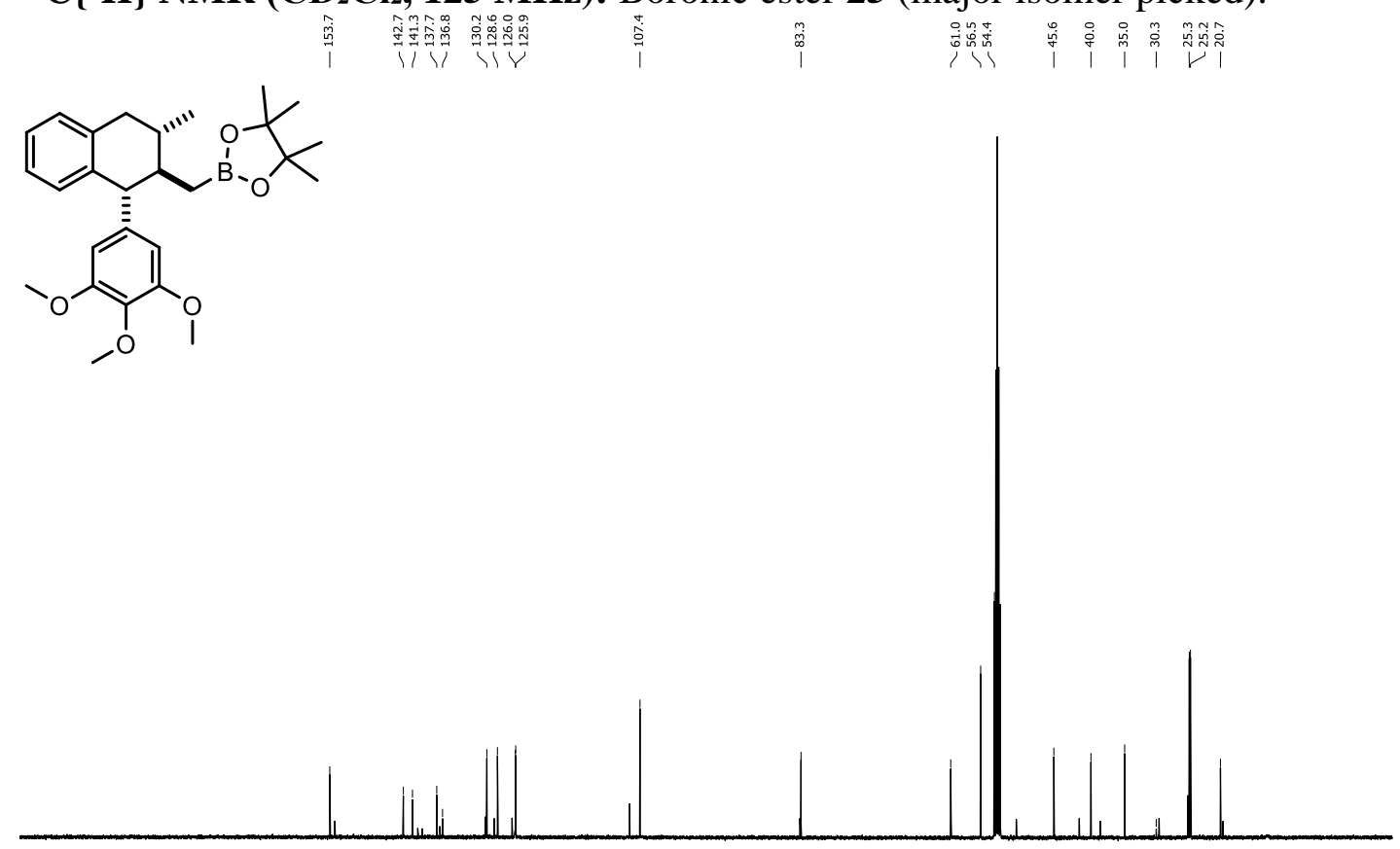

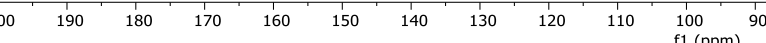

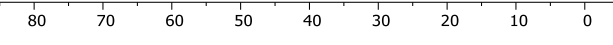


${ }^{13} \mathrm{C}\left\{{ }^{1} \mathrm{H}\right\}$ NMR $\left(\mathrm{CD}_{2} \mathrm{Cl}_{2}, 125 \mathrm{MHz}\right)$ : Boronic ester 25 (minor isomer picked).<smiles>COc1cc(C2c3ccccc3C[C@@H](C)[C@H]2COC(C)(C)C(C)(C)C)cc(OC)c1OC</smiles>
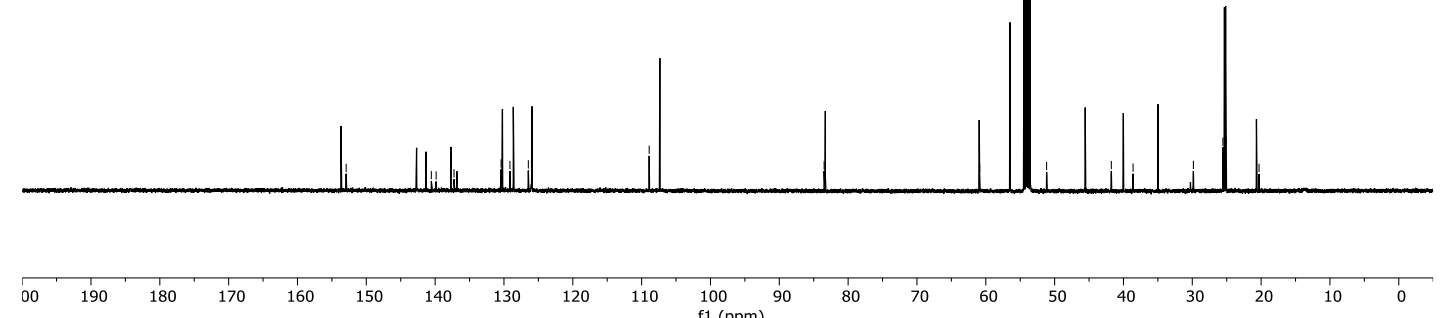

${ }^{1} \mathrm{H}$ NMR ( $\mathrm{CD}_{2} \mathrm{Cl}_{2}, 600 \mathrm{MHz}$ ): Boronic ester 26 (major isomer picked).

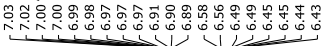

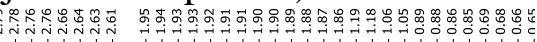<smiles>COc1ccc([C@H]2c3ccccc3C[C@@H](C)[C@H]2COC(C)(C)C(C)(C)C)c(OC)c1</smiles>

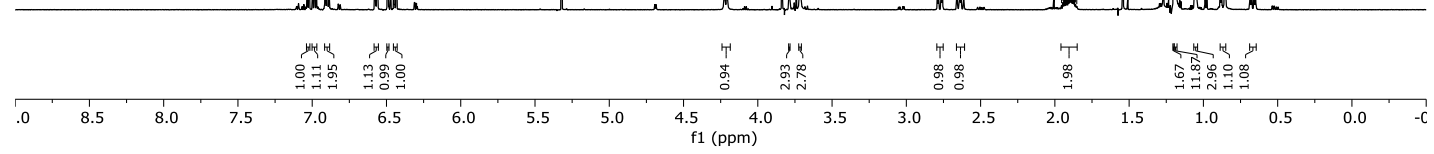


${ }^{1} \mathrm{H}$ NMR $\left(\mathrm{CD}_{2} \mathrm{Cl}_{2}, 600 \mathrm{MHz}\right)$ : Boronic ester 26 (minor isomer picked).

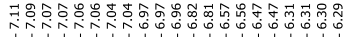

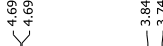

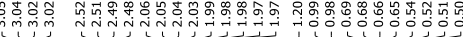<smiles>CCc1ccccc1C(COC(C)(C)C)c1ccc(OC)cc1OC</smiles>

H. Ill.

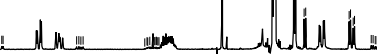

wh" ph:

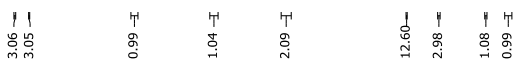

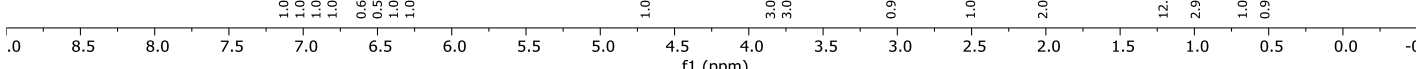

${ }^{13} \mathrm{C}\left\{{ }^{1} \mathrm{H}\right\}$ NMR ( $\left.\mathrm{CD}_{2} \mathrm{Cl}_{2}, 150 \mathrm{MHz}\right)$ : Boronic ester 26 (major isomer picked).

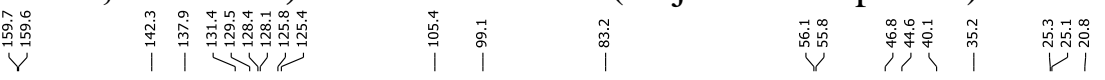<smiles>COc1ccc([C@H]2c3ccccc3C[C@@H](C)[C@H]2CB2OC(C)(C)C(C)(C)O2)c(OC)c1</smiles>

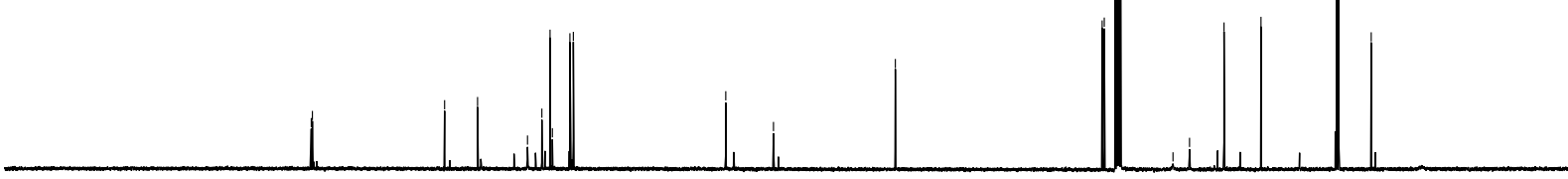

00

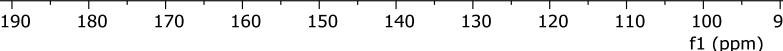


${ }^{13} \mathrm{C}\left\{{ }^{1} \mathrm{H}\right\}$ NMR $\left(\mathrm{CD}_{2} \mathrm{Cl}_{2}, 150 \mathrm{MHz}\right)$ : Boronic ester 26 (minor isomer picked).

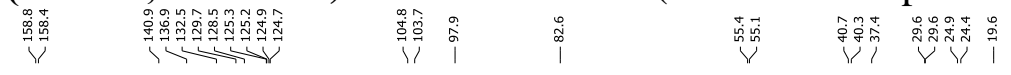<smiles>COc1ccc(C2c3ccccc3C[C@H](C)C2CB2OC(C)(C)C(C)(C)O2)c(OC)c1</smiles>
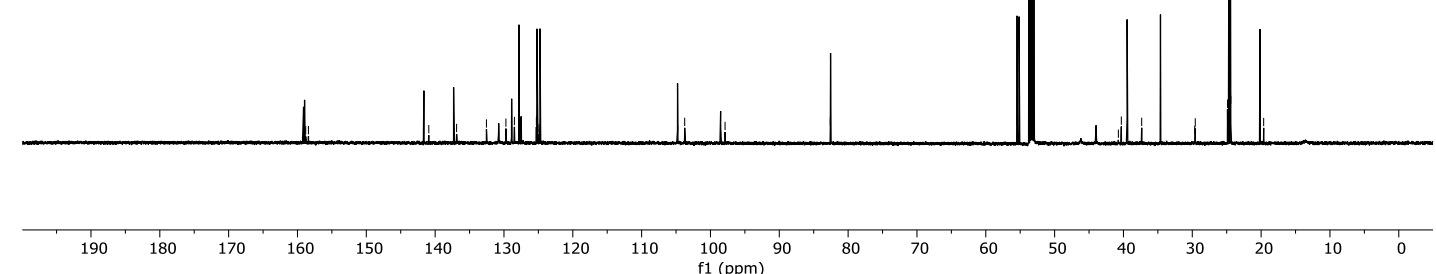

${ }^{1} \mathrm{H}$ NMR ( $\left.\mathrm{CD}_{2} \mathrm{Cl}_{2}, 600 \mathrm{MHz}\right)$ : Boronic ester 27 (major isomer picked).

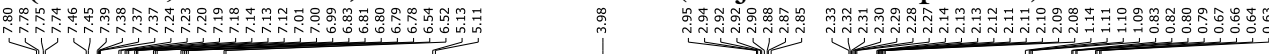

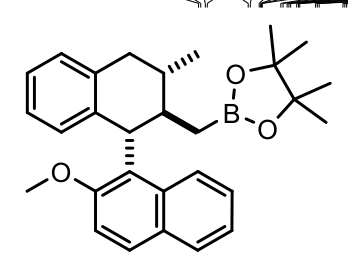

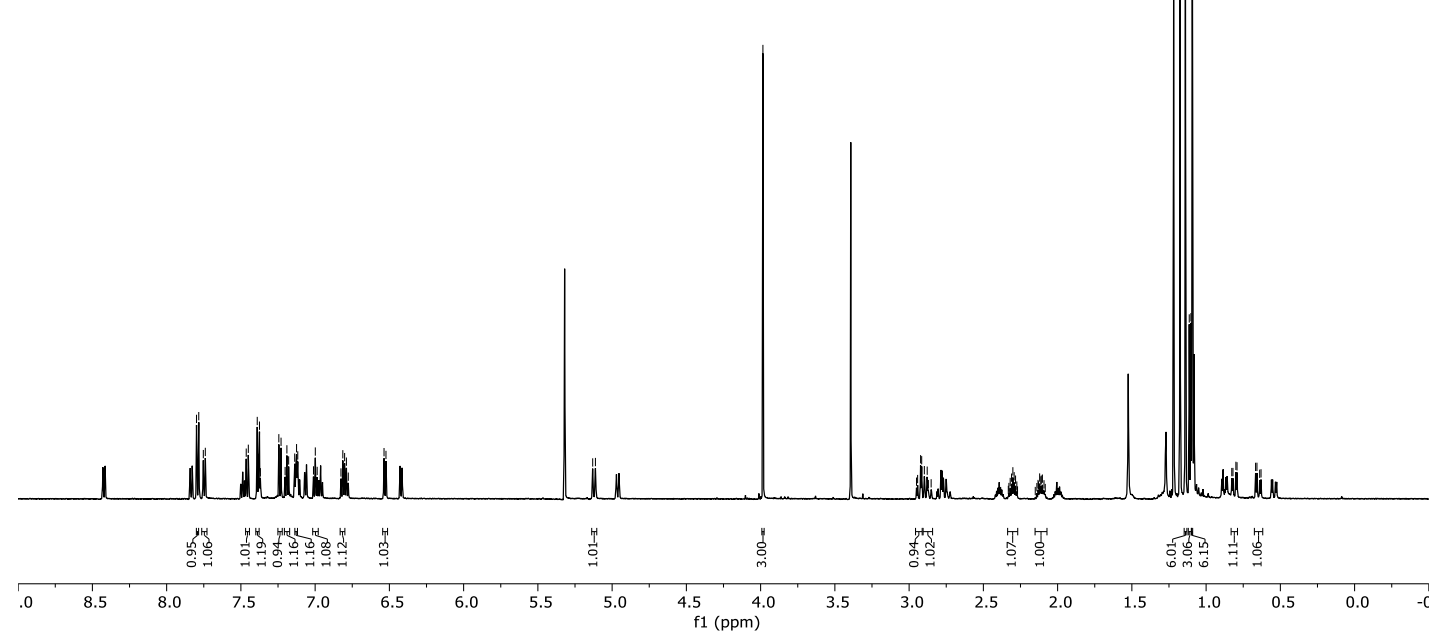


${ }^{1} \mathrm{H}$ NMR ( $\left.\mathrm{CD}_{2} \mathrm{Cl}_{2}, 600 \mathrm{MHz}\right)$ : Boronic ester 27 (minor isomer picked).

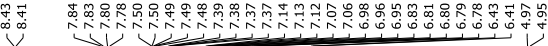

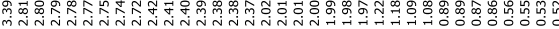<smiles>COc1ccc2ccccc2c1C1c2ccccc2C[C@H](C)C1CB1OC(C)(C)C(C)(C)O1</smiles>

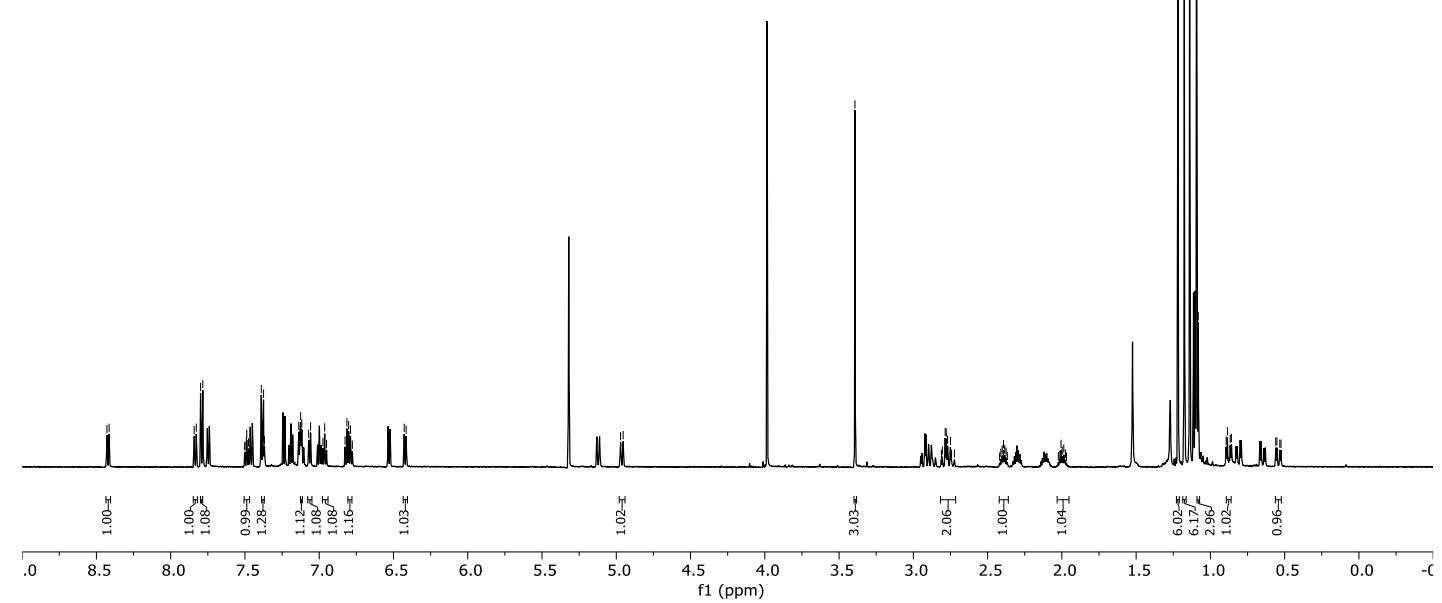

${ }^{13} \mathrm{C}\left\{{ }^{1} \mathrm{H}\right\}$ NMR $\left(\mathrm{CD}_{2} \mathrm{Cl}_{2}, 150 \mathrm{MHz}\right)$ : Boronic ester 27 (major and minor isomers picked).

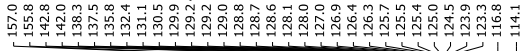

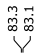

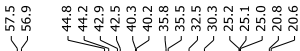<smiles>COc1ccc2ccccc2c1[C@H]1c2ccccc2C[C@H](C)C1CB1OC(C)(C)C(C)(C)O1</smiles><smiles>COc1ccc2ccccc2c1C1c2ccccc2C[C@H](C)C1CB1OC(C)(C)C(C)(C)O1</smiles> 
${ }^{1} \mathrm{H}$ NMR ( $\mathrm{CD}_{2} \mathrm{Cl}_{2}, 500 \mathrm{MHz}$ ): Boronic ester 28 (major isomer picked).
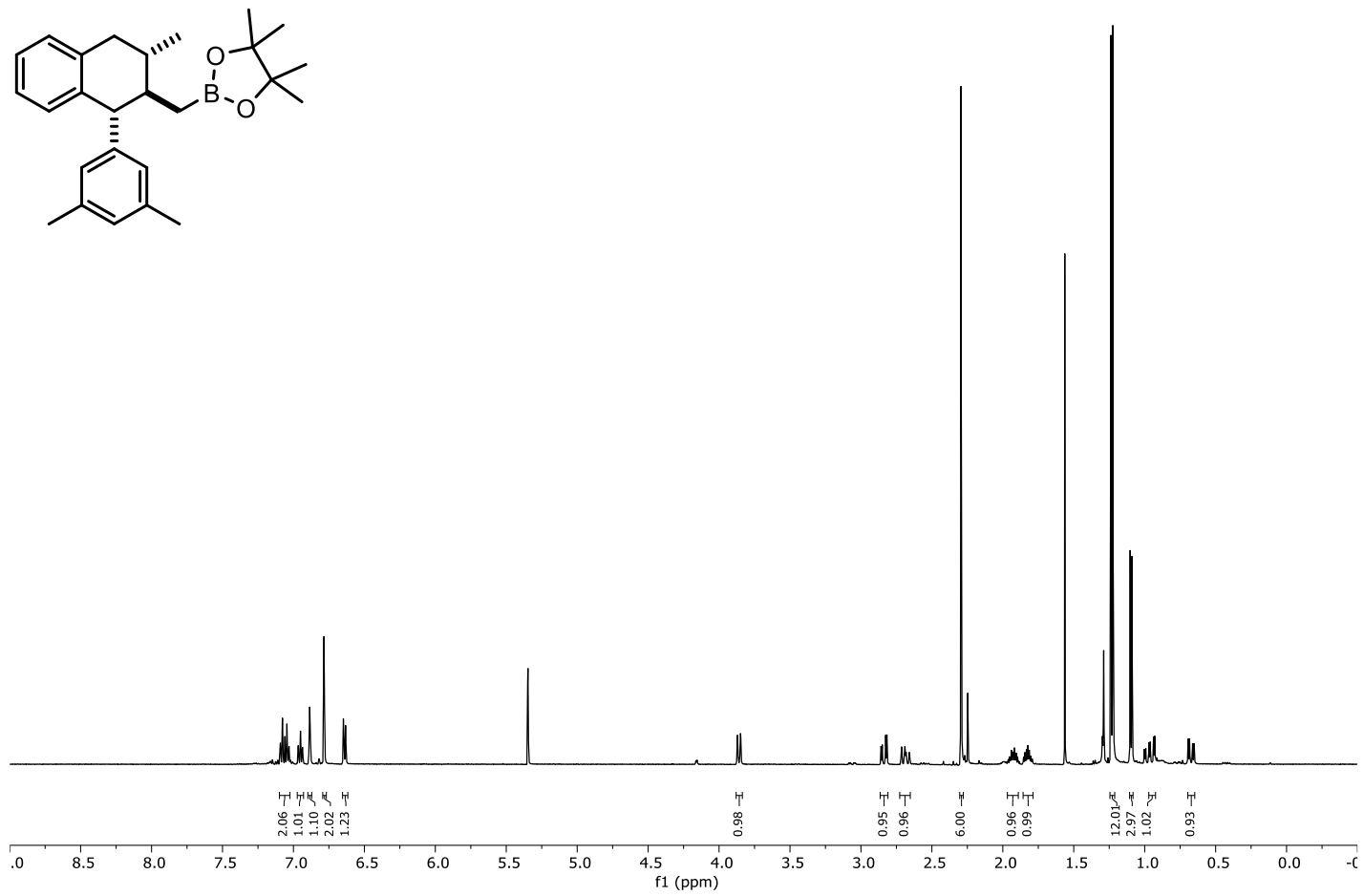

${ }^{13} \mathrm{C}\left\{{ }^{1} \mathrm{H}\right\}$ NMR $\left(\mathrm{CD}_{2} \mathrm{Cl}_{2}, 125 \mathrm{MHz}\right)$ : Boronic ester 28 (major isomer picked).
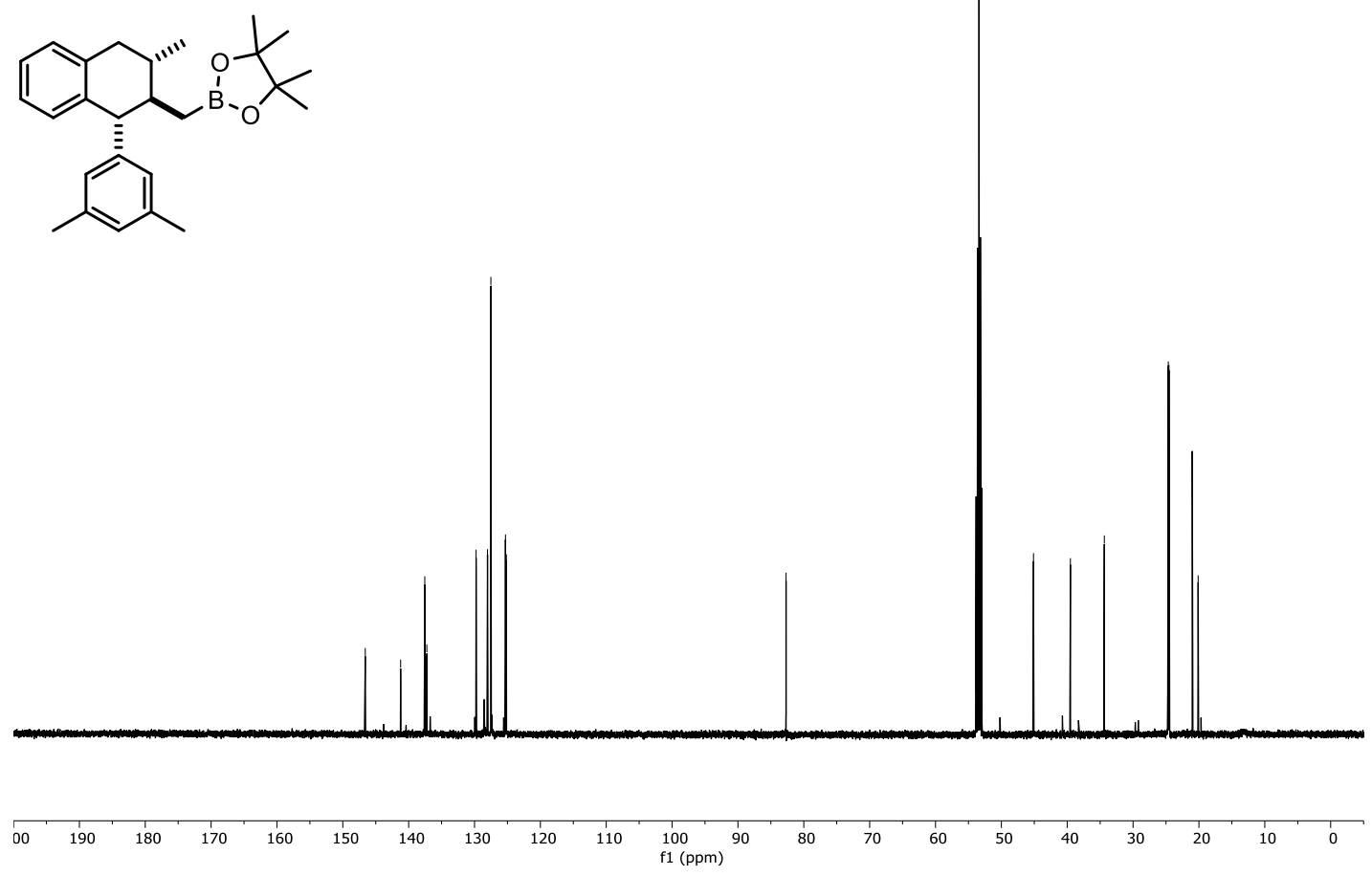

46 
${ }^{1} \mathrm{H}$ NMR ( $\left.\mathrm{CD}_{2} \mathrm{Cl}_{2}, 600 \mathrm{MHz}\right)$ : Boronic ester 29 (major isomer picked).

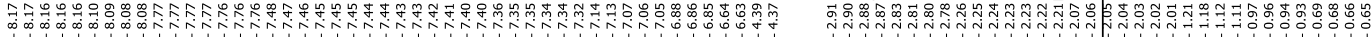<smiles>C[C@H]1Cc2ccccc2[C@H](c2cccc3c2sc2ccccc23)C1CB1OC(C)(C)C(C)(C)O1</smiles>

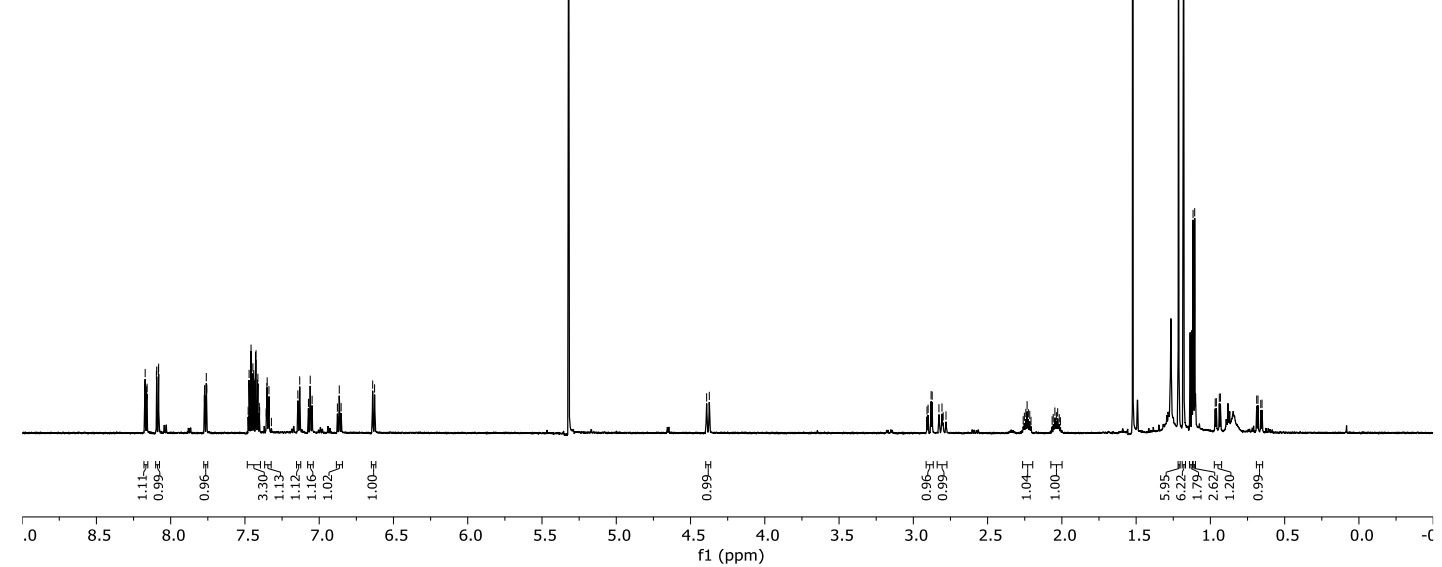

${ }^{1} \mathrm{H}$ NMR $\left(\mathrm{CD}_{2} \mathrm{Cl}_{2}, 600 \mathrm{MHz}\right)$ : Boronic ester 29 (minor isomer picked).

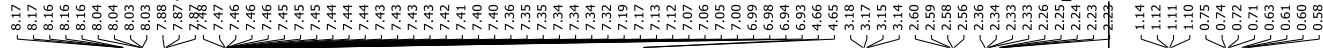<smiles>C[C@H]1Cc2ccccc2C(c2cccc3c2sc2ccccc23)C1CB1OC(C)(C)C(C)(C)O1</smiles>

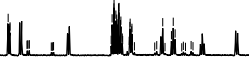
Nonum und

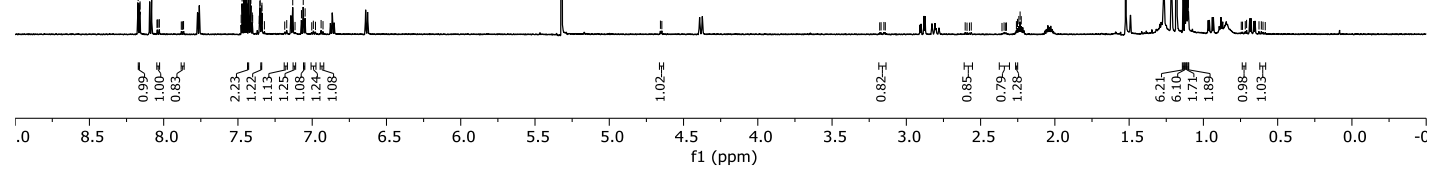


${ }^{13} \mathrm{C}\left\{{ }^{1} \mathrm{H}\right\}$ NMR $\left(\mathrm{CD}_{2} \mathrm{Cl}_{2}, 150 \mathrm{MHz}\right)$ : Boronic ester 29 (major and minor isomer picked).

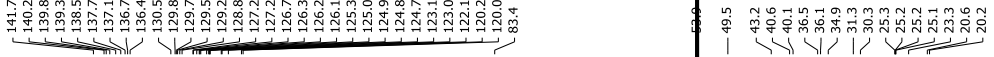

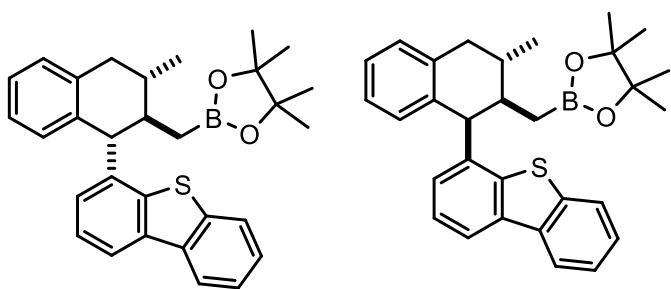

${ }^{1} \mathrm{H}$ NMR $\left(\mathrm{CD}_{2} \mathrm{Cl}_{2}, 600 \mathrm{MHz}\right)$ : Liganoid 30 (major isomer picked).

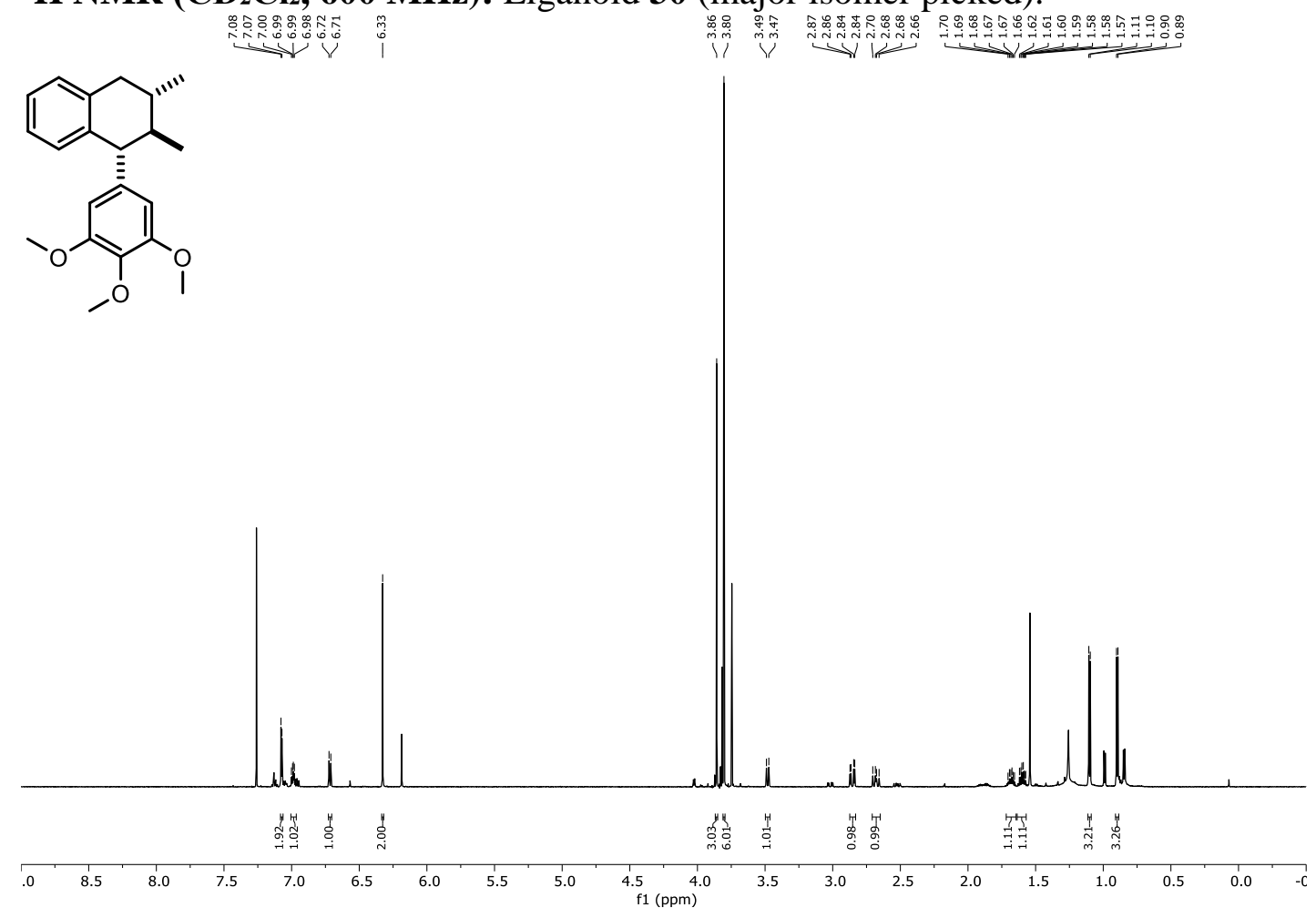


${ }^{1} \mathrm{H}$ NMR ( $\left.\mathrm{CD}_{2} \mathrm{Cl}_{2}, 600 \mathrm{MHz}\right)$ : Liganoid 30 (minor isomer picked).

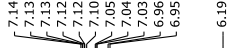

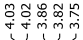

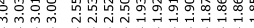<smiles>COc1cc(C2c3ccccc3CC(C)C2C)cc(OC)c1OC</smiles>

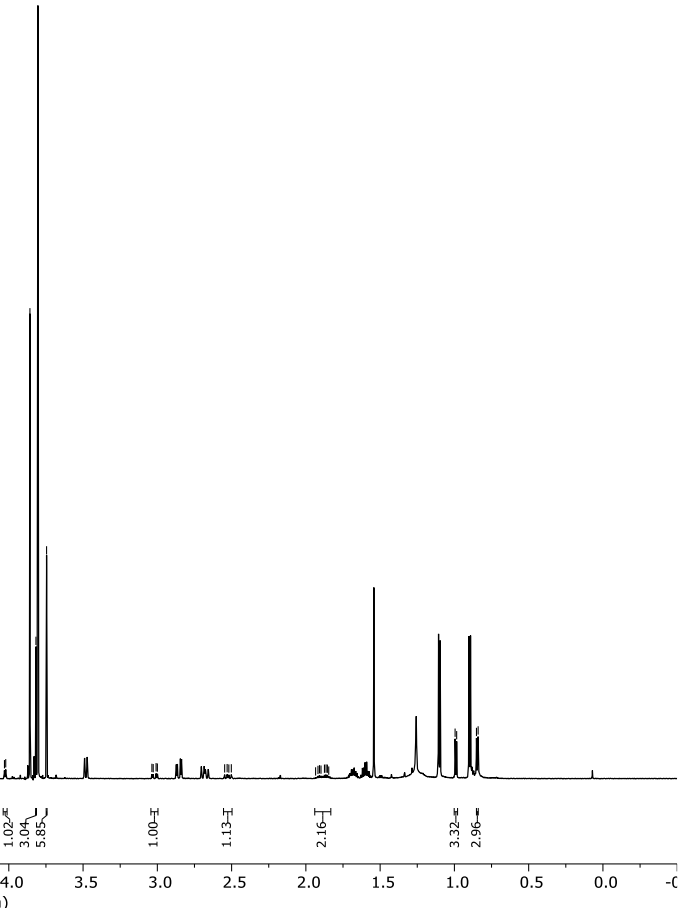

${ }^{13} \mathrm{C}\left\{{ }^{1} \mathrm{H}\right\}$ NMR $\left(\mathrm{CD}_{2} \mathrm{Cl}_{2}, 150 \mathrm{MHz}\right)$ : Lignanoid 30 (major isomer picked).

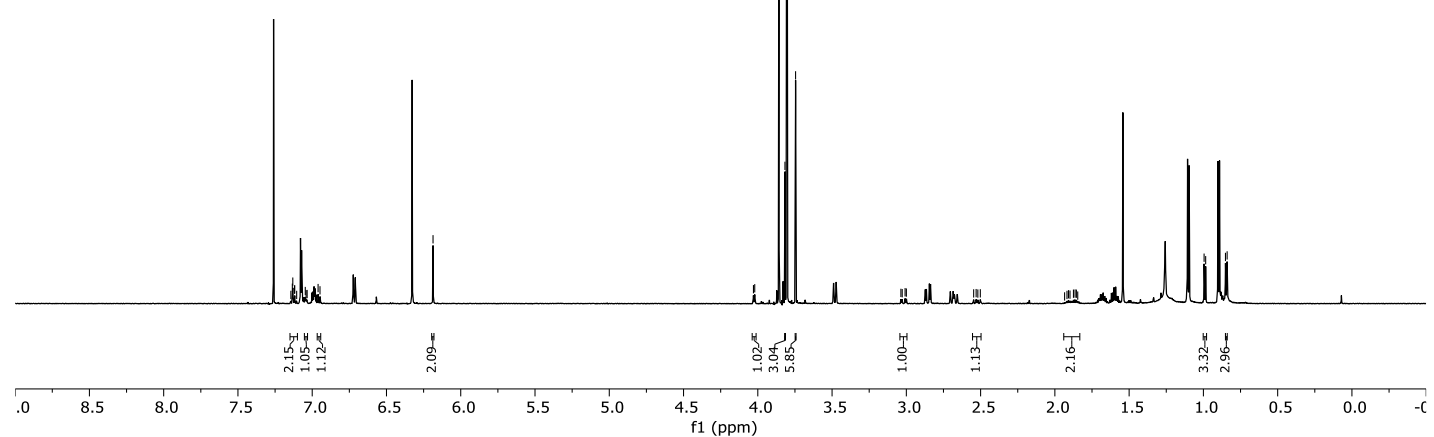<smiles>COc1cc([C@H]2c3ccccc3C[C@H](C)C2C)cc(OC)c1OC</smiles>

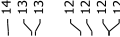

焉


${ }^{13} \mathrm{C}\left\{{ }^{1} \mathrm{H}\right\}$ NMR $\left(\mathrm{CD}_{2} \mathrm{Cl}_{2}, 150 \mathrm{MHz}\right)$ : Lignanoid 30 (minor isomer picked).

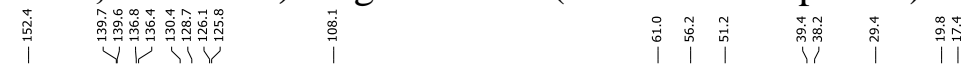<smiles>COc1cc(C2c3ccccc3C[C@H](C)C2C)cc(OC)c1OC</smiles>

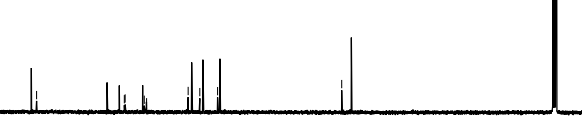

${ }^{1} \mathrm{H}$ NMR $\left(\mathrm{CD}_{2} \mathrm{Cl}_{2}, 600 \mathrm{MHz}\right)$ : Liganoid 31 (major isomer picked).

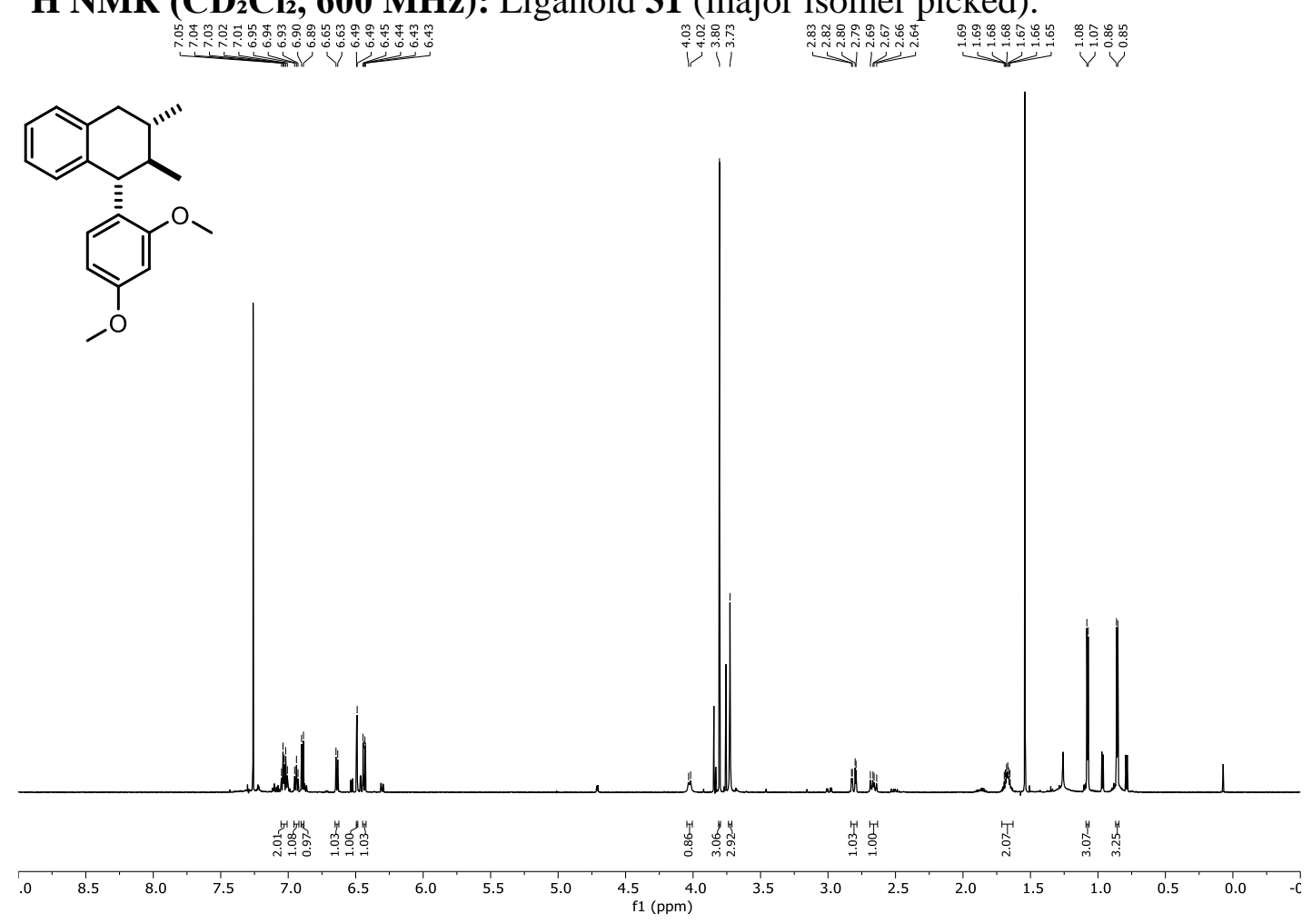


${ }^{1} \mathrm{H}$ NMR ( $\left.\mathrm{CD}_{2} \mathrm{Cl}_{2}, 600 \mathrm{MHz}\right)$ : Liganoid 31 (minor isomer picked).

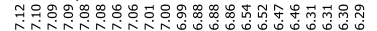

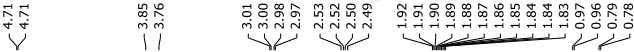<smiles>COc1ccc(C2c3ccccc3C[C@H](C)C2C)c(OC)c1</smiles>

Whathill

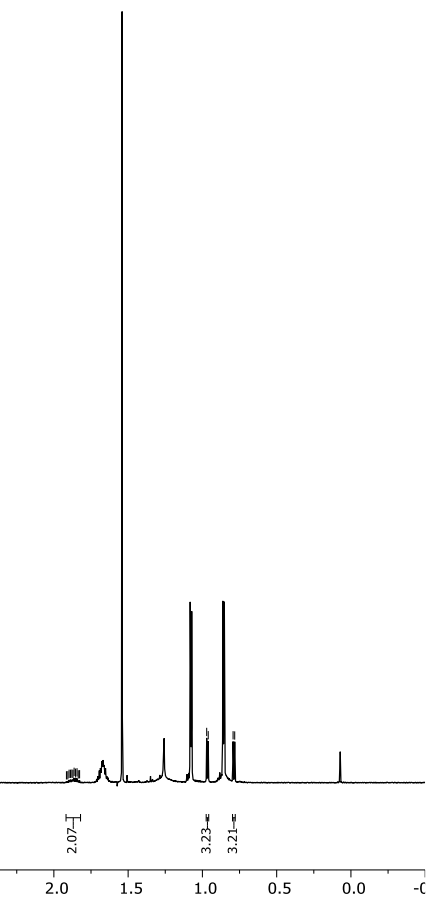

${ }^{13} \mathrm{C}\left\{{ }^{1} \mathrm{H}\right\}$ NMR $\left(\mathrm{CD}_{2} \mathrm{Cl}_{2}, 150 \mathrm{MHz}\right)$ : Lignanoid 31 (major and minor isomer picked).

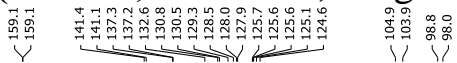

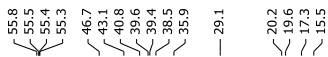<smiles>COc1ccc([C@H]2c3ccccc3C[C@@H](C)C2C)c(OC)c1</smiles><smiles>COc1ccc(C2c3ccccc3C[C@H](C)C2C)c(OC)c1</smiles>
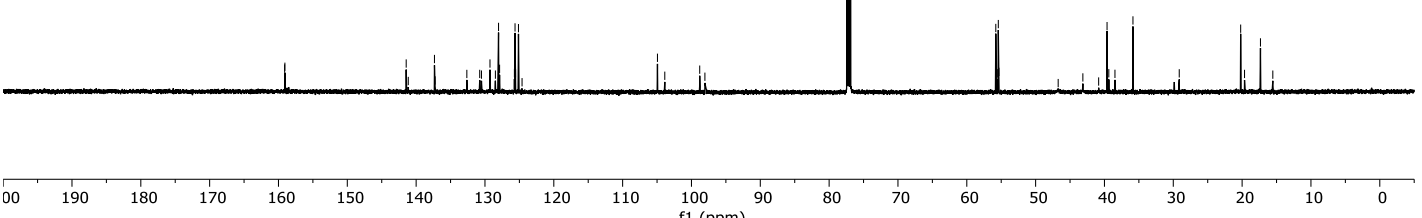
${ }^{1} \mathrm{H}$ NMR ( $\mathrm{CD}_{2} \mathrm{Cl}_{2}, 600 \mathrm{MHz}$ ): Liganoid 32 (major isomer picked).

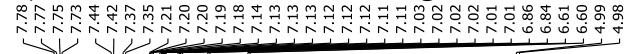

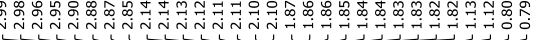<smiles>COc1ccc2ccccc2c1[C@H]1c2ccccc2C[C@H](C)C1C</smiles>

$\stackrel{\longrightarrow}{\longrightarrow}$

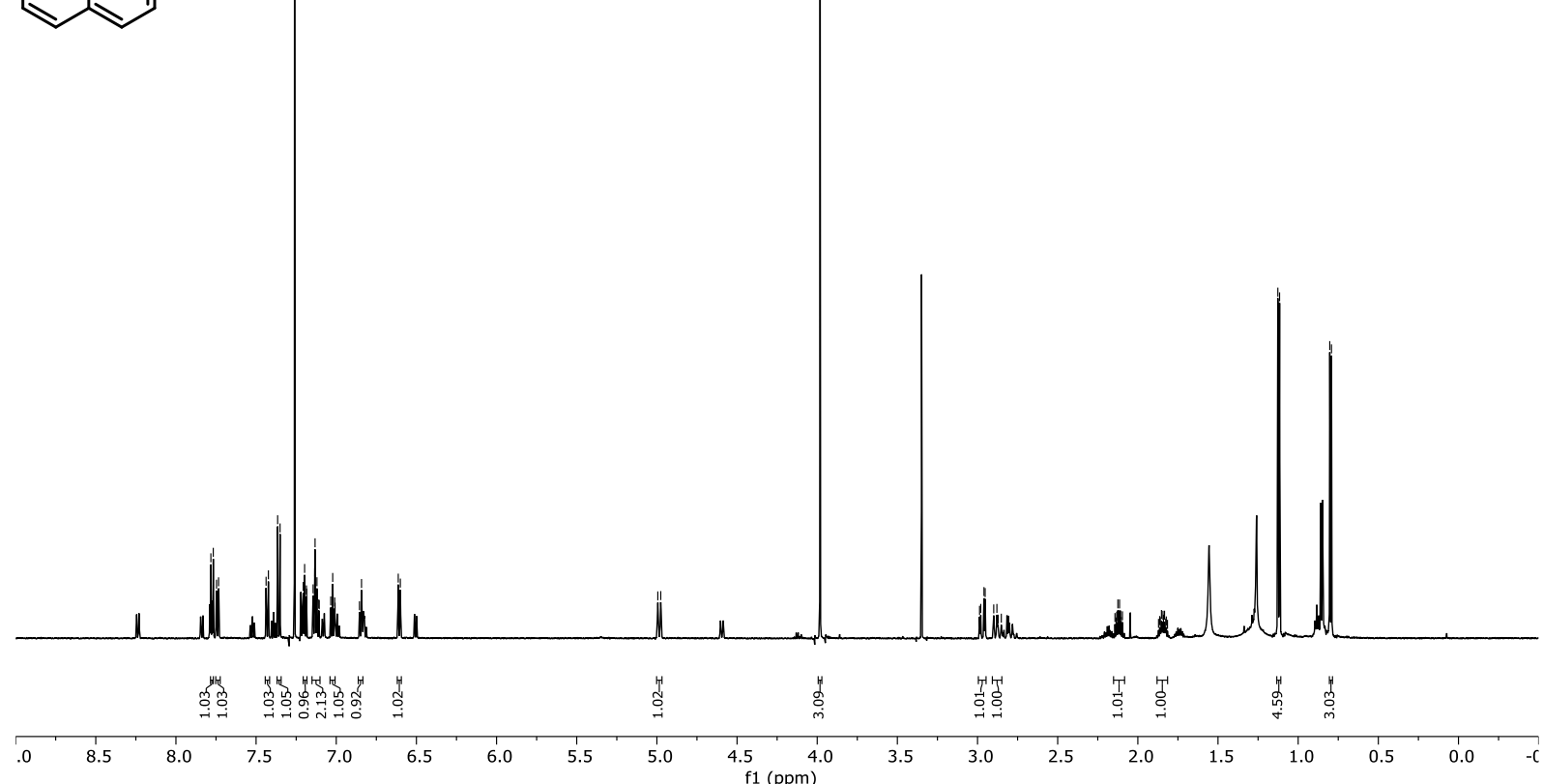

${ }^{1} \mathrm{H}$ NMR ( $\mathrm{CD}_{2} \mathrm{Cl}_{2}, 600 \mathrm{MHz}$ ): Liganoid 32 (minor isomer picked).

等

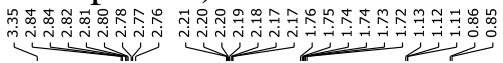

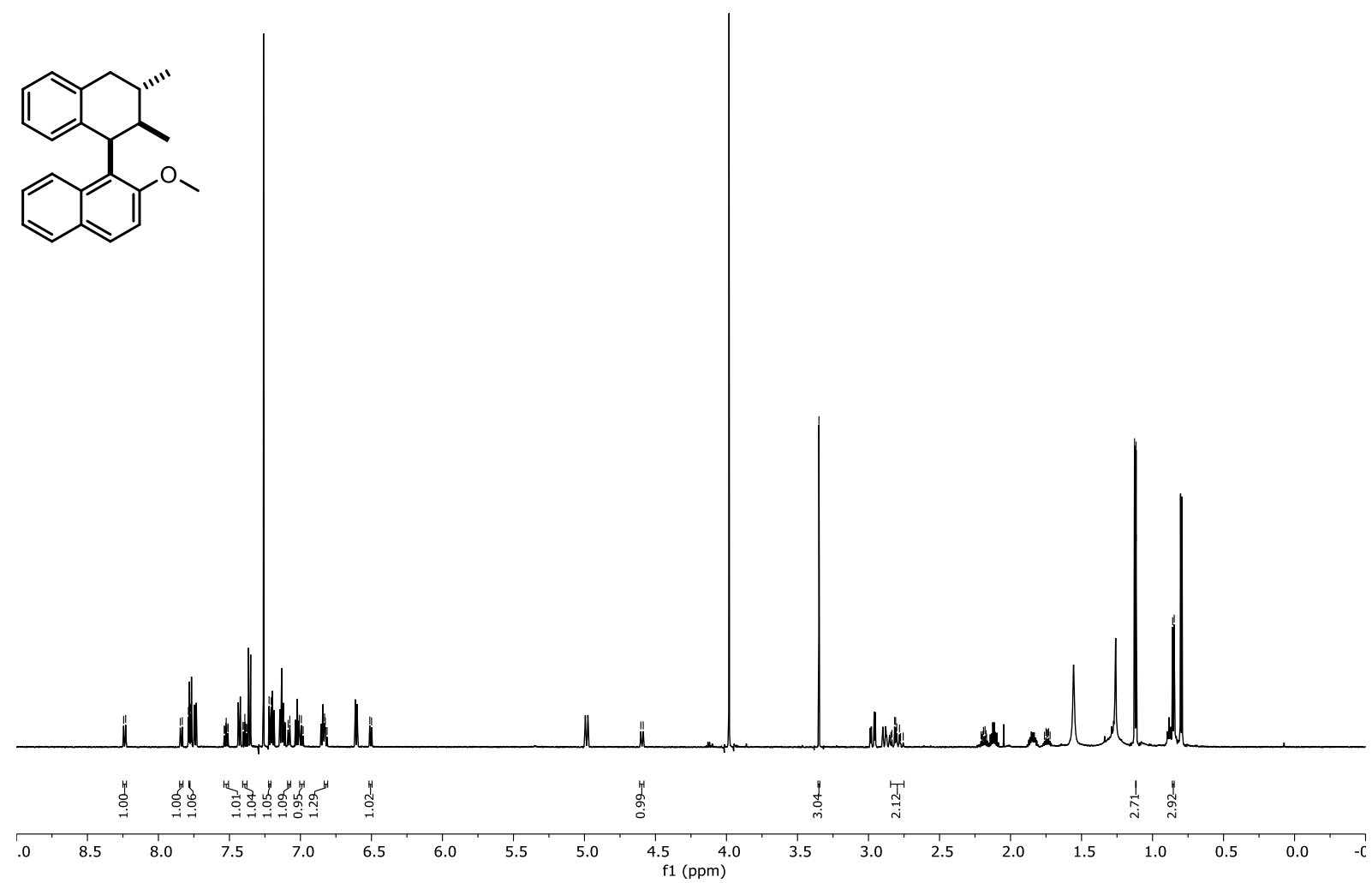


${ }^{13} \mathrm{C}\left\{{ }^{1} \mathrm{H}\right\}$ NMR $\left(\mathrm{CD}_{2} \mathrm{Cl}_{2}, 150 \mathrm{MHz}\right)$ : Lignanoid 32 (major and minor isomer picked).

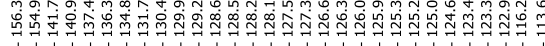

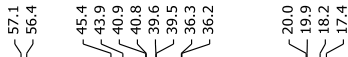<smiles></smiles><smiles>COc1ccc2ccccc2c1C1c2ccccc2C[C@H](C)C1C</smiles>

${ }^{1} \mathrm{H}$ NMR ( $\left.\mathrm{CD}_{2} \mathrm{Cl}_{2}, 600 \mathrm{MHz}\right)$ : Liganoid 33.

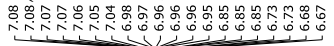

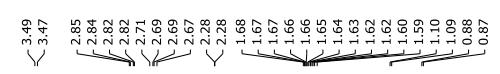<smiles>Cc1cc(C)cc([C@H]2c3ccccc3C[C@H](C)C2C)c1</smiles>

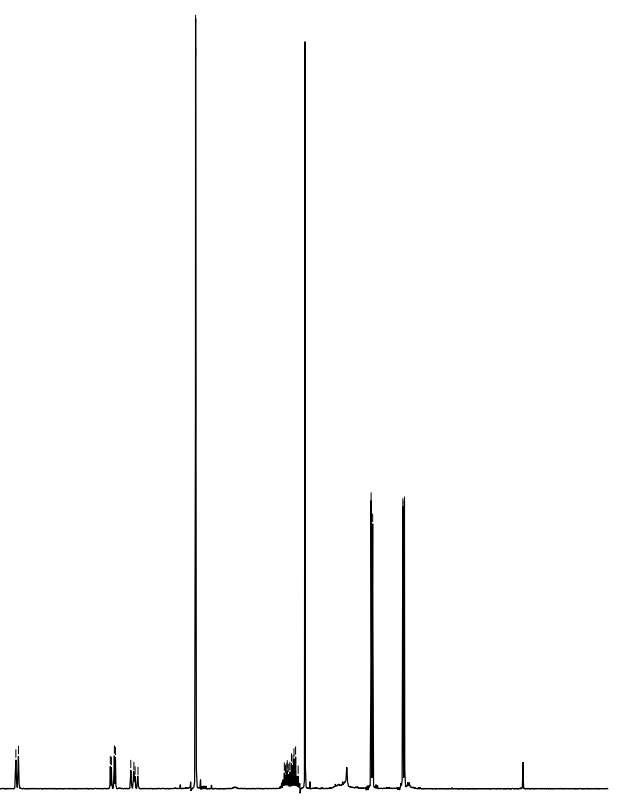

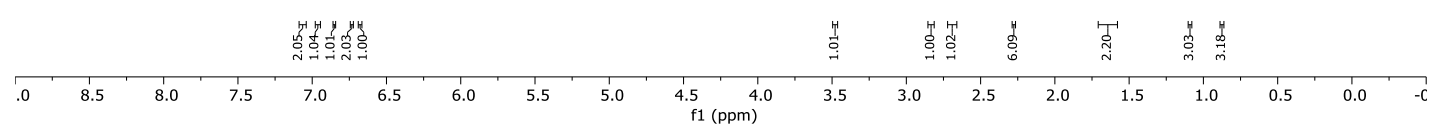


${ }^{13} \mathrm{C}\left\{{ }^{1} \mathrm{H}\right\}$ NMR $\left(\mathrm{CD}_{2} \mathrm{Cl}_{2}, 150 \mathrm{MHz}\right):$ Lignanoid 33.

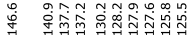

(1)

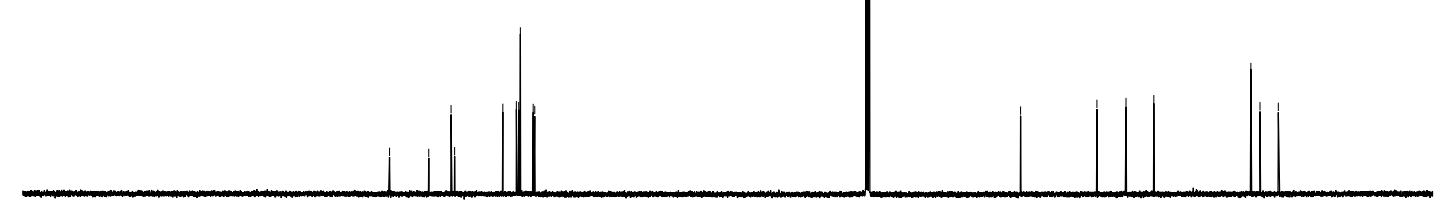

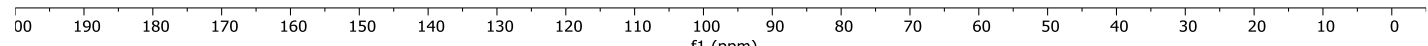

${ }^{1} \mathrm{H}$ NMR $\left(\mathrm{CD}_{2} \mathrm{Cl}_{2}, 600 \mathrm{MHz}\right)$ : Liganoid 34 (major isomer picked).

定

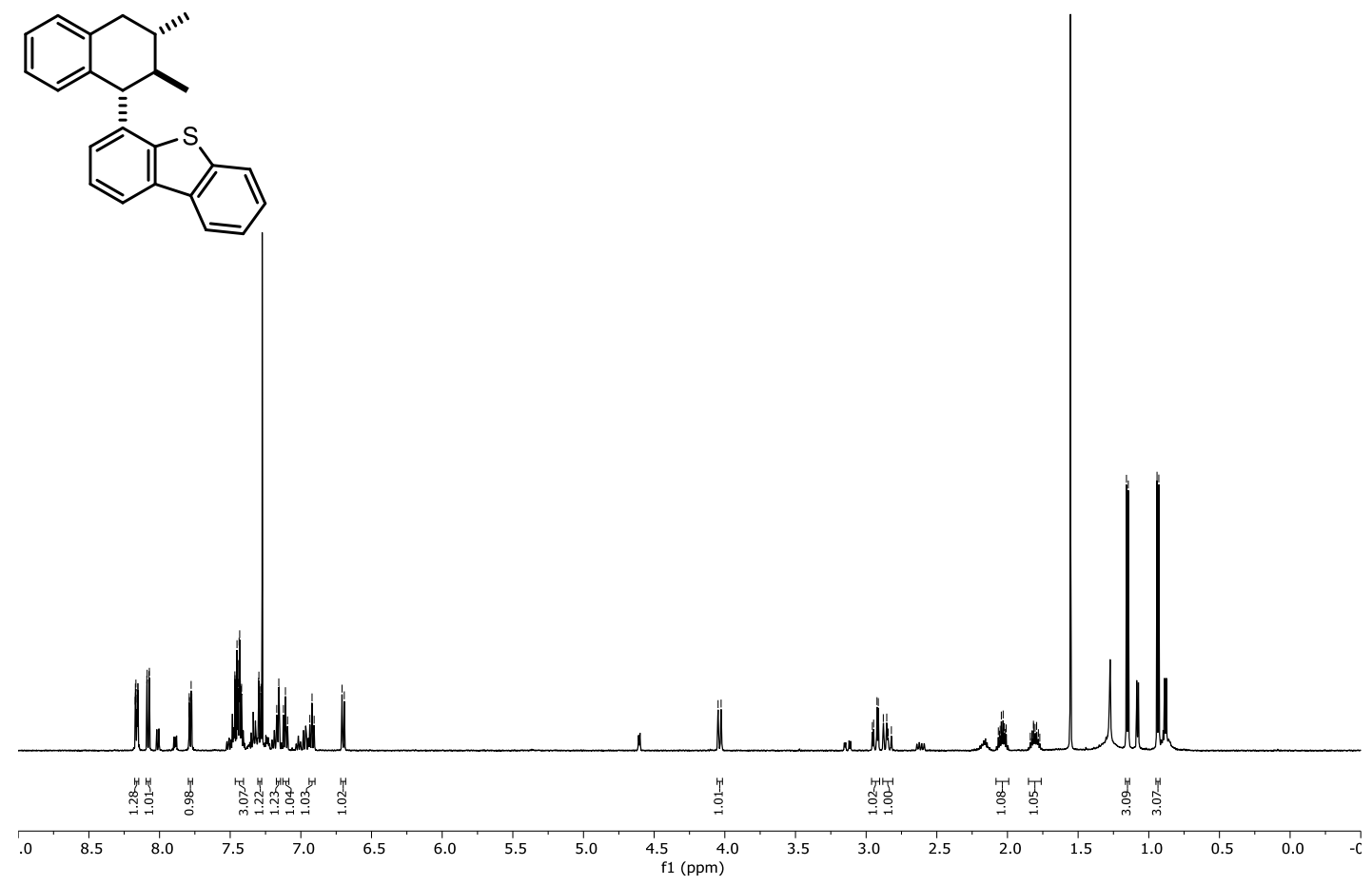


${ }^{1} \mathrm{H}$ NMR ( $\left.\mathrm{CD}_{2} \mathrm{Cl}_{2}, 600 \mathrm{MHz}\right)$ : Liganoid 34 (minor isomer picked).

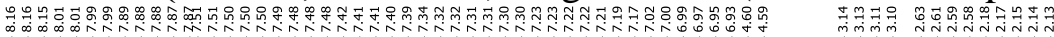

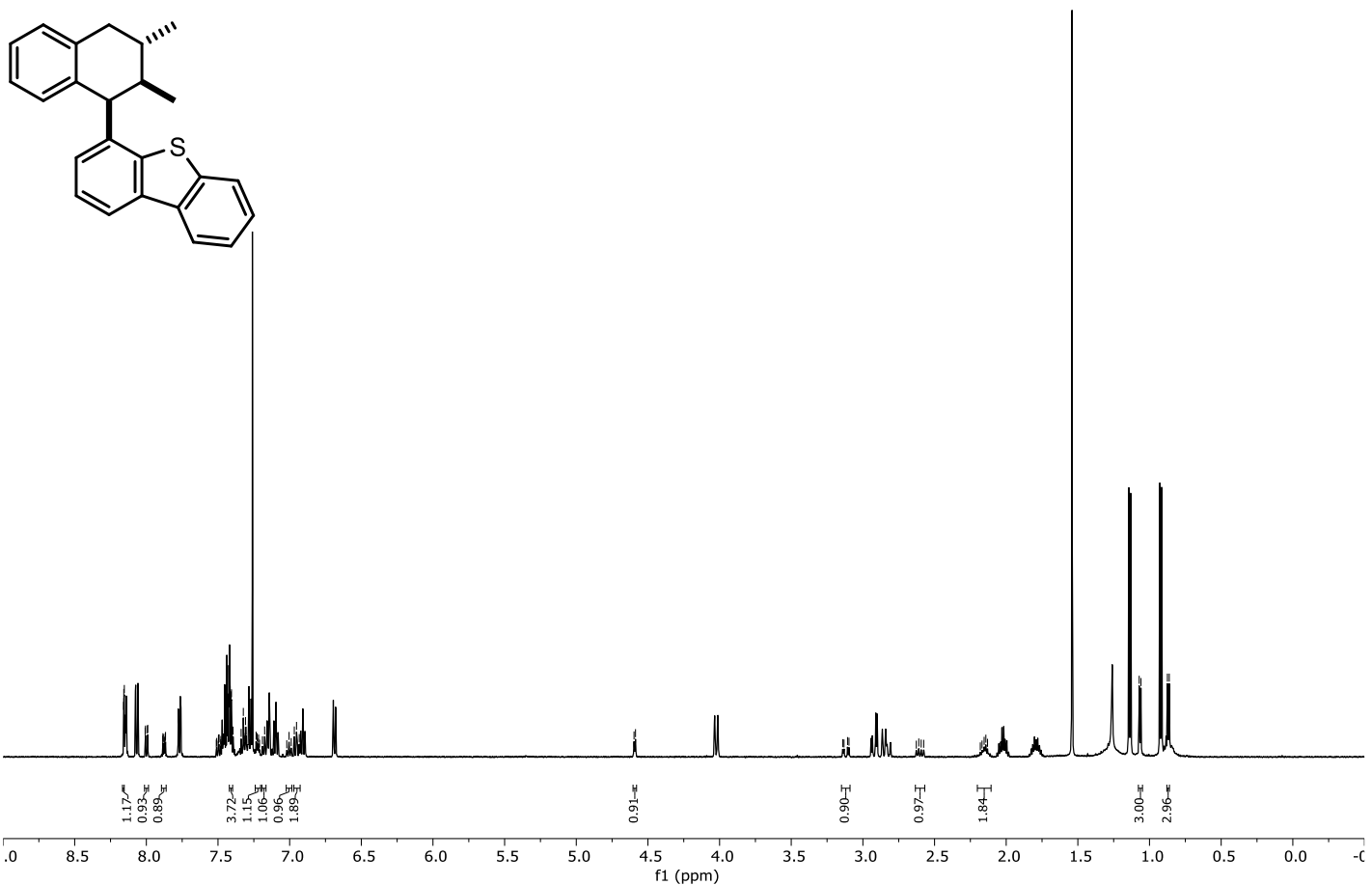

${ }^{13} \mathrm{C}\left\{{ }^{1} \mathrm{H}\right\}$ NMR $\left(\mathrm{CD}_{2} \mathrm{Cl}_{2}, 150 \mathrm{MHz}\right)$ : Lignanoid 34 (major and minor isomer picked).<smiles>C[C@H]1Cc2ccccc2[C@H](c2cccc3c2sc2ccccc23)[C@H]1C</smiles>

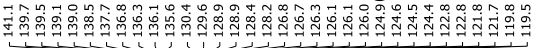


(1) Wang, L.; Prabhudas, B.; Clive, D. L. J. J. Am. Chem. Soc. 2009, 131 (16), 6003-6012.

(2) Yamada, H.; Hirayama, F.; Koshio, H.; Matsumoto, Y.; Yanagisawa, I. Production of Naphthalene Derivative and Its Production Intermediate. JP2000219665 (A), August 8, 2000.

(3) Teague, S. J.; Roth, G. P. Synthesis 1986, 1986 (05), 427-429.

(4) Do, Q.-Q. T.; Guo, C.; Humphries, P. S.; Marakovits, J. T.; Dong, L.; Hou, X.; Johnson, M. C. Benzimidazole or Indole Amides as Inhibitors of Pin1. WO2006040646A1, April 20, 2006.

(5) Hong, B. C.; Hsu, C. S.; Lee, G. H. Chem. Commun. 2012, 48, 2385-2387.

(6) Rawson, D. J.; Meyers, A. I. J. Org. Chem. 1991, 56 (7), 2292-2294. 